\title{
MILITARIA DE GAULE MÉRIDIONALE, 19. LE MOBILIER MILITAIRE ROMAIN DANS LE DÉPARTEMENT DE L'HÉRAULT (F)
}

POR

\author{
MiCHEL FEUGÈRE
}

\section{RÉSUMÉ - RESUMEN}

L'inventaire des mobiliers militaires romains d'un département français du littoral méditerranéen, l'Hérault, fait apparaître une série de plus de 200 objets, dispersés sur les sites d'habitat et échelonnés dans le temps de la République au Bas-Empire. Ces objets sont analysés dans leur répartition, leur fonction et leur chronologie, afin de proposer un modèle interprétatif prenant en compte les plus récents acquis de la recherche dans ce domaine. On propose un schéma mixte, privilégiant pour la période la mieux représentée, le Haut-Empire, des objets rapportés par les recrues levées en masse dans la province, notamment pour la cavalerie du Ier siècle. Cette hypothèse n'exclut pas la possibilité d'autres sources de diffusion de ce mobilier, comme la présence épisodique, notamment dans les agglomérations, de détachements militaires chargés de missions diverses.

El inventario de los objetos muebles militares romanos de un Departamento del litoral mediterráneo francés, el Hérault, arroja una serie de más de doscientos objetos, repartidos en diferentes yacimientos y escalonados en el tiempo desde época republicana al Bajo Imperio. Analizamos estos objetos según su dispersión, su función y su cronología, con el fin de proponer un modelo interpretativo que tenga en cuenta los más recientes hallazgos de la investigación en este campo. Se propone un esquema mixto, que enfatiza para el período mejor representado (el Alto Imperio) la presencia de objetos asociados a los reclutamientos en masa en la Provincia, y en especial la caballería del s. I d.C. Esta hipótesis no excluye la posibilidad de otras fuentes de difusión de estos objetos, como la presencia ocasional, especialmente en las aglomeraciones, de destacamentos militares encargados de misiones diversas.

\section{MOTS CLÉS - PALABRAS CLAVE}

Militaria. Narbonnaise. sites civils. habitats. harnachement. cingulum. vétérans.

Militaria. Narbonense. Asentamientos civiles. Habitat. Arreos. Cingulum. Veteranos.

\section{INTRODUCTION}

Il y a vingt ans, à la suite d'un séjour en Grande-Bretagne qui m'avait permis de découvrir l'archéologie militaire, j'avais tenté un premier inventaire territorial des militaria de France en dressant un catalogue des armes et pièces d'équipement militaire romain du département de la Loire (Feugère 1983). Tout incomplète qu'elle fût, cette liste présente au moins l'intérêt de fournir un point de repère lorsque l'on tente de comparer les trouvailles d'objets militaires en Gaule civile, ce que nous avons fait avec Matthieu Poux à l'occasion du XIIIe colloque ROMEC de Vindonissa, en 2001 (Feugère, Poux 2001). 
Depuis le début des années 80 , la situation de l'archéologie nationale a largement évolué. C'est d'abord le développement des fouilles, notamment de sauvetage, qui a radicalement modifié la documentation, apportant des masses parfois considérables de mobilier généralement bien daté. Mais c'est aussi, et rien ne sert de l'occulter, la multiplication exponentielle des utilisateurs de détecteurs de métaux, qui apporte des informations bien différentes: difficilement accessibles et parfois peu fiables, lorsqu'il s'agit d'objets conservés chez des particuliers; mais associés à des données de terrain précises et bien disséminées sur le territoire, lorsqu'elles proviennent de prospections autorisées menées par des équipes reconnues.

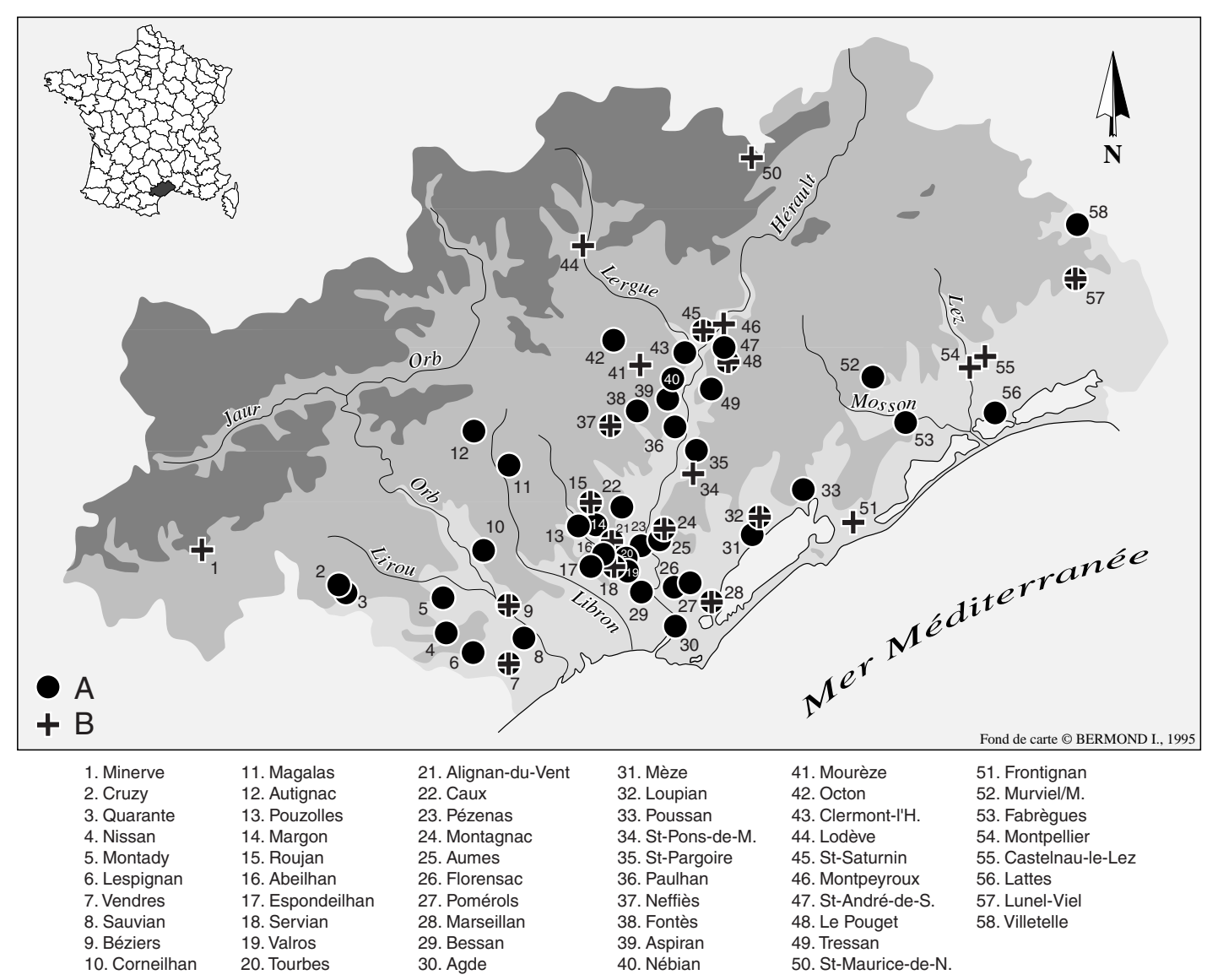

Fig. 1. Département de l'Hérault: localisation des sites ayant livré du mobilier militaire; A: Haut-Empire; B: Bas-Empire.

À ce renouvellement de la documentation s'ajoute depuis quelques années un regain considérable de l'intérêt des archéologues pour le mobilier militaire: participent à ce mouvement, en bonne partie suscité par des chercheurs anglo-saxons, les ROMEC (Roman Military Equipment Conferences) les Limeskongresse et des revues comme le Journal of Roman Military Equipment Studies. Il n'est que temps de disposer de corpus qui permettent, d'un côté, de connaître le mobilier disponible dans chaque région, et d'autre part, d'établir les comparaisons nécessaires avec les sites les mieux connus sous ce rapport. C'est ce que nous tentons ici, pour un département assez bien connu du Midi de la France, l'Hérault. À mi-chemin du Rhône et 
des Pyrénées, ce département du littoral languedocien se partage entre une plaine densément occupée et un arrière-pays plus difficile, souvent recouvert par des garrigues ou des maquis (fig. 1). Dans l'Antiquité, il se partage entre les cités romaines de Béziers, Lodève et Nîmes.

Les militaria de ce département ont fait l'objet de quelques publications ponctuelles (Feugère 1996a; Feugère, Pellecuer 1998), ainsi que de la mise en perspective évoquée cidessus. Mais leur catalogue définitif n'avait pu être publié jusqu'à maintenant: c'est le but de cet article, qui couvre l'ensemble des objets connus de l'auteur en 2002, du Haut- et du BasEmpire. Le catalogue est présenté, site par site, dans l'ordre alphabétique des communes; les illustrations, qui reproduisent autant d'objets que possible, même s'ils ont déjà été publiés, privilégient ainsi l'association des trouvailles issues d'un même site, ce que ne permettaient pas les études, surtout typologiques, parues jusqu'ici sur cette documentation.

\section{TYPOLOGIE ET INTERPRÉTATION}

\subsection{Questions de sélection}

Si le mobilier militaire demeure peu étudié en contexte civil, et notamment en Gaule, c'est sans doute parce que la détermination du mobilier que l'on pourrait attribuer à l'armée romaine demeure, en-dehors d'un contexte clairement militaire, des plus délicate. Le XIIIe colloque ROMEC (Vindonissa, 2001), consacré à ce thème, a clairement mis en évidence les difficultés de cette démarche. Bien sûr, chaque spécialiste, confronté à un corpus d'objets, fait ses propres choix. Mais les difficultés commencent lorsque l'on veut comparer, d'un site à l'autre, des collections sélectionnées selon des critères différents. La nature des objets attestés, mais aussi et peut-être surtout les proportions des différentes catégories entre elles (armes, cingulum, harnachement...). À titre d'exemple, les petits boutons circulaires en bronze, largement utilisés sur les tabliers de cingulum, me paraissent difficiles à distinguer d'autres décors pouvant avoir concerné des véhicules ou de l'ameublement. Nous ne les avons donc pas pris en compte sur les sites héraultais.

\subsection{Catégories et formes principales}

\subsubsection{Les armes}

Tout en restant rares, elles sont relativement bien attestées dans le département, avec $11 \%$ des objets pris en compte dans ce corpus. Si les formes anciennes des armatures de traits d'artillerie font défaut dans la documentation actuelle, on compte sur quelques sites indigènes (oppida comme Aumes ou Magalas, mais aussi un port - Lattes - et un site beaucoup plus modeste, Pézenas), de rares balles de fronde en plomb. Isolées, celles-ci ne peuvent guère être attribuées à des assauts en règle, comme on l'observe sur les sites provençaux où de telles batailles sont en revanche bien attestées (Willaume 1987; Chabot, Feugère 1993; Arnaud 1999); leur présence sur ces sites pose néanmoins le problème de leur attribution et de leur utilisation, militaire ou non (Feugère 1992). On notera pourtant, sur l'oppidum de Magalas, l'association troublante d'une balle de fronde en plomb et d'une pointe de flèche en fer à trois ailettes, objet typiquement militaire.

Les casques italo-étrusques, du type à bouton sommital ou à calotte lisse (type CoolusMannheim) sont relativement nombreux dans l'Hérault, comme sur le reste du littoral sudgaulois. Leur présence dans deux tombes indigènes du département voisin (Nîmes et Saint- 
Laurent-des-Arbres) $)^{1}$ montre ces armes ne doivent pas être nécessairement associées à un statut de militaire romain. Des casques italiques ont, de la même manière, été déposés dans des puits, autrefois dénommés funéraires, et considérés aujourd'hui comme des ensembles votifs indigènes (Vidal 1991; Boudet 1996). Si le contexte du casque de Fabrègues demeure inconnu, les deux fragments de casques récemment découverts à Aumes et à Marseillan semblent suggérer, avec d'autres exemples languedociens, que ces objets pouvaient être utilisés sur les habitats.

Sous l'Empire, la documentation se limite à de rares pointes de lance et quelques fragments de glaives, ou plutôt de leurs fourreaux, ainsi que de très rares éléments de cuirasse. Ce mobilier semble résulter de situations assez diverses. Les pointes de lance ont pu servir à la chasse, comme le montre le texte du fameux «Testament du Lingon» ou, en Languedoc, le mobilier d'une tombe inédite de Narbonne. Leur présence sur de riches villae (Pézenas, Loupian...) s'accorde bien avec cette interprétation. Peut-on interpréter de la même manière les quelques fragments de fourreaux connus dans le département (bouterolle d'Aumes, barrette de Mèze)? Si le glaive fait, lui aussi, partie des armes de chasse mentionnées par le Testament du Lingon, il s'agit aussi d'une arme dont la possession devait être sérieusement contrôlée sous l'Empire. On a noté que les fusées de poignées de glaives ne se rencontrent pratiquement, en Gaule du Sud, que dans les villes (Nîmes, Vaison), ce qui semble étayer l'hypothèse de possesseurs militaires (passages de troupes, ou corps occasionnels de maintien de l'ordre) (Feugère 1997).

La cuirasse segmentée est, au contraire, une protection très clairement limitée à certaines troupes d'élite de l'infanterie. Un fragment de Clermont-l'Hérault, vicus associé à un nœud routier et sans doute à un important marché, appartient à un modèle du IIe s. particulièrement rare en Gaule.

\subsubsection{Le cingulum}

En-dehors des boucles et plaques les plus caractéristiques, l'attribution de diverses pièces au cingulum ou au harnais pose de sérieux problèmes d'identification. C'est notamment le cas des boucles simples, en D, que l'on a pris l'habitude de décrire comme «boucles de cingulum»; or, en reproduisant le mobilier d'une banale écurie (stabulum) de la Maison de Ménandre à Pompei, M. Mackensen a très judicieusement rappelé la grande variété des boucles utilisées dans le harnachement des chevaux romains, qu'il s'agisse des chevaux montés ou attelés (Mackensen 2001) (fig. 2). Il nous faut donc, sur cette indication, revenir sur un certain nombre de boucles attribuées jusqu'ici au cingulum et qui semblent bien appartenir au harnachement, avec pour conséquence un net accroissement de cette catégorie qui était déjà clairement majoritaire (v. infra et fig. 7).

Restent néanmoins dans la catégorie du cingulum les boucles les plus travaillées, comme celles de la villa de la Savoye à Vendres, ou encore les plaques rectangulaires caractéristiques (Murviel, Pézenas, Villetelle). Ces objets indiscutablement militaires posent, à eux seuls, le problème des contacts entre les fournisseurs des armées et le monde civil, dans un contexte où le cingulum constituait à lui seul le symbole d'un statut prestigieux dans la Narbonnaise du Ier siècle, celui de militaire (en dernier lieu: Obmann 1999). Les diverses interprétations de ce mobilier en contexte civil ont été récemment discutées à la ROMEC de Vindonissa, en 2001.

1 La tombe de Nîmes, Mas de Vignol, est une découverte récente (fouilles V. Bel, 2001; rens. N. Chardenon): le type exact du casque (avec ou sans bouton), retrouvé écrasé sur place, ne peut être précisé au moment où ces lignes sont rédigées. La tombe de Saint-Laurent-des-Arbres est mieux connue (Barruol, Sauzade 1969); elle a été sensiblement vieillie lors d'un rééexamen des chronologies funéraires de Gaule du Sud (Bats 1990) et date probablement des alentours de 100 av. n. ère, voire du dernier quart du IIe s. av. n. ère. Selon les découvertes de R. Boudet (1996), le type à calotte lisse remplacerait définitivement le type à bouton sommital au moment de la réforme de Marius, dans les dernières années du IIe s. av. n. ère. 


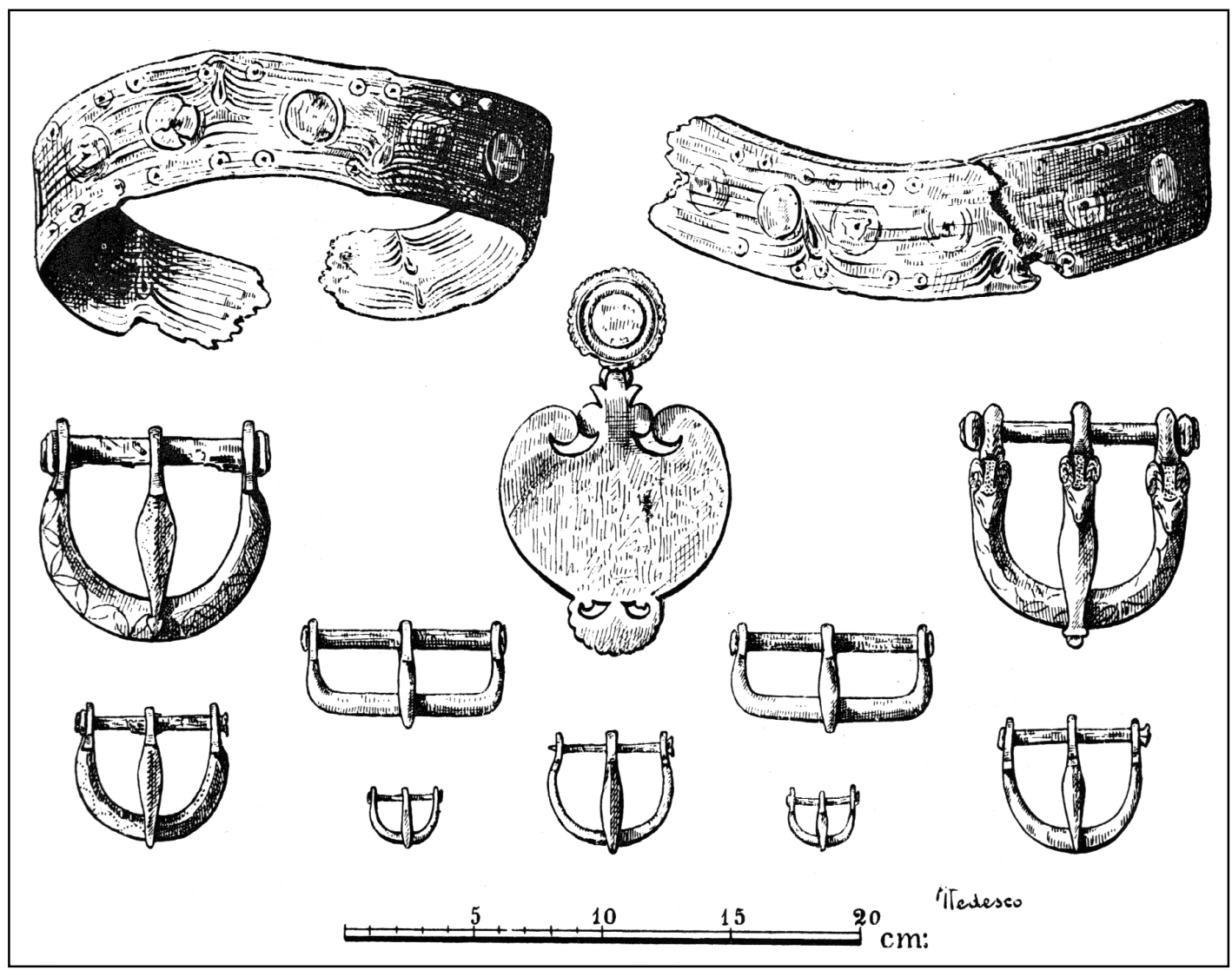

Fig. 2. Militaria d'une écurie de Pompei (d'ap. A. Maiuri, reproduit par Mackensen 2001).

\subsubsection{Le harnachement}

On ne trouve dans l'Hérault qu'une seule phalère certaine (Caux, $n^{\circ} 32$ ), autrefois pourvue d'un décor niellé tout à fait classique pour ces objets, mais aussi deux pieces décorées dont l'attribution demeure équivoque ( $\mathrm{n}^{\circ} 101$ et 114 , harnais ou décoration militaire?). Le système de pendant articulé sur une phalère paraît avoir été très peu répandu en Gaule du Sud (v. infra les différents types de pendants), sans que l'on puisse trouver à ce phénomène d'autre explication que la raréfaction des recrutements méridionaux à partir de la deuxième moitié du Ier siècle.

Les pendants à crochets, qui précèdent chronologiquement les pendants à charnière, forment la très grande majorité du corpus héraultais: cette proportion est représentative de l'ensemble de la Gaule du Sud, au sens large, où l'on observe une grande variété de formes (fig. 3). Comme on le verra plus loin, différentes appliques, notamment émaillées, illustrent néanmoins la permanence du harnais décoré aux IIe et IIIe siècles: l'abondance des pendants à crochet n'implique donc pas nécessairement que les harnais de Gaule méridionale datent majoritairement de cette période. Une classification de ces objets a été proposée il y a quelques années par M.C. Bishop (1988), et E. Deschler-Erb a ensuite étudié plus précisément les pendants à crochet de type Bishop 7 (Deschler-Erb 1998a et b). Si l'étude des pendants à crochets permet de suivre diverses séries apparemment typiques, il n'en va pas de même des objets plus tardifs, parmi lesquels on observe une nette diversification typologique. 


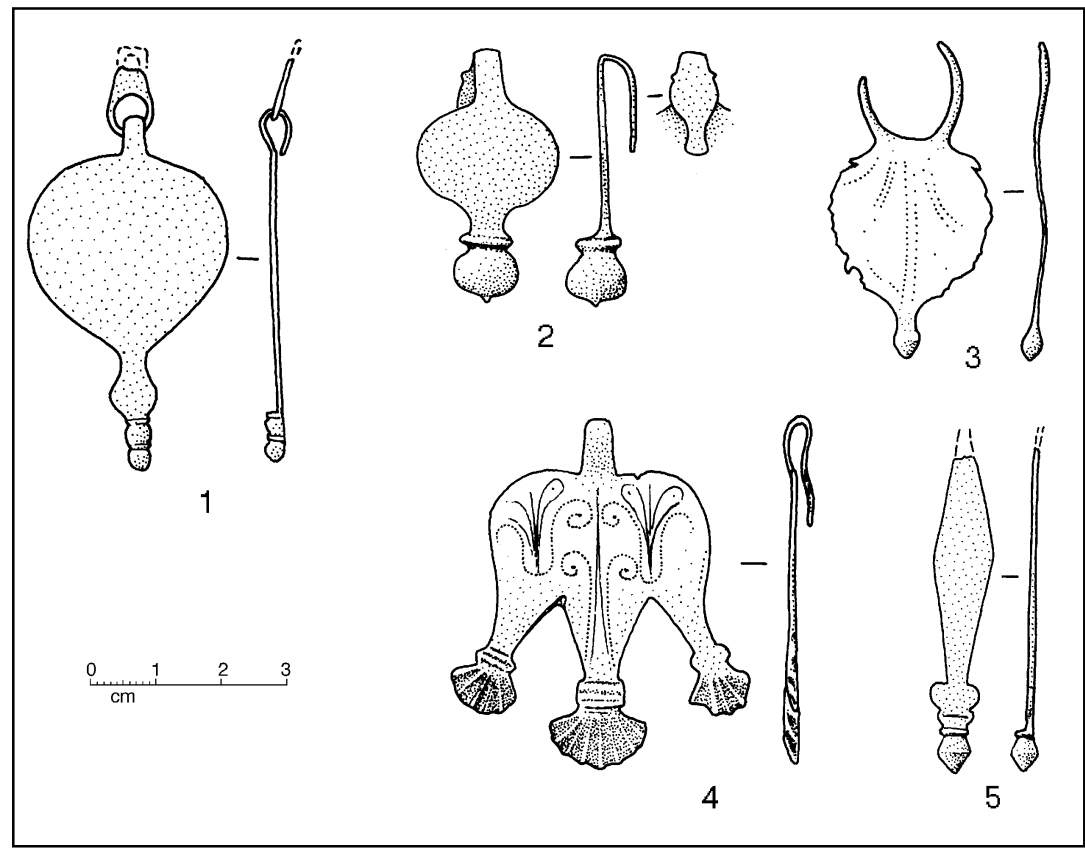

Fig. 3. Pendants de harnais à crochet de Gaule méridionale: 1 et $2, \mathrm{Al}-$ bias, Cos (Tarn-et-Garonne) (anc. coll. M. Elmi); 3 et 4, «Gaule méridionale»; 5, Laure-Minervois (coll. C. Journet). Ech. 2/3.

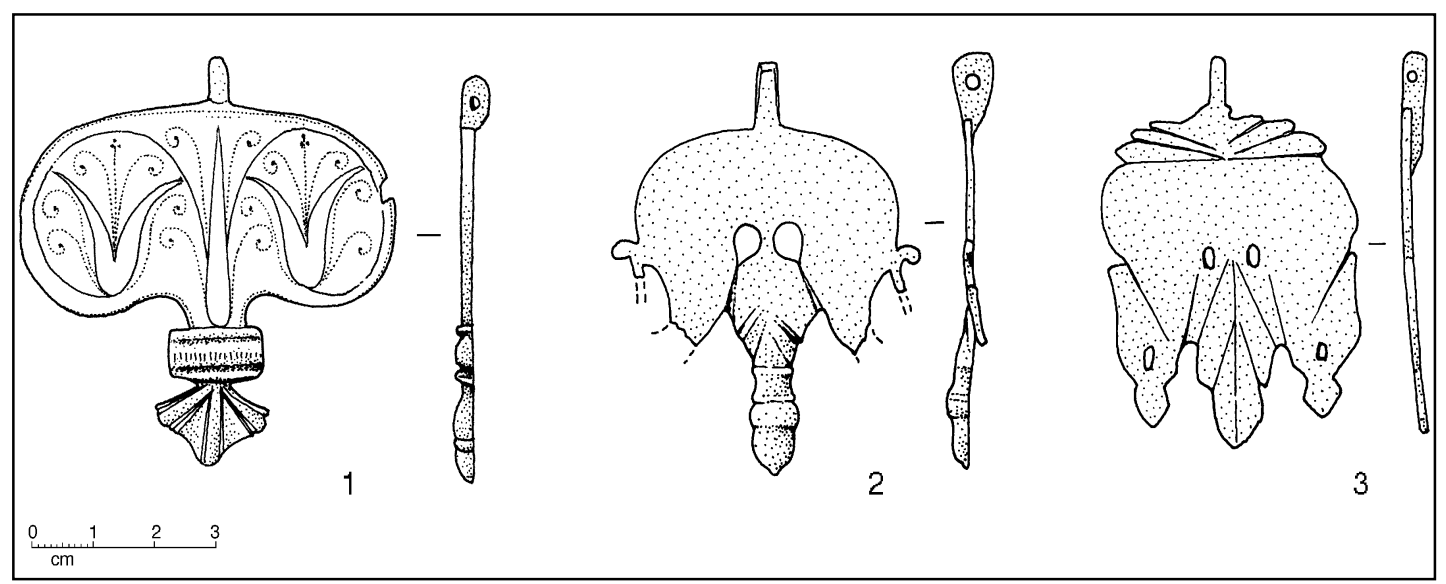

Fig. 4. Pendants de harnais à charnière de Gaule du Sud et de l'Ouest: 1, Guimps (Char.Maritime), d'ap. J.-L. Tilhard; 2, Albias, Cos (Tarn-et-Garonne), anc. coll. M. Elmi; 3, le Langon, salle polyvalente (Vendée), fouilles E. Bernard. Ech. 2/3.

La rareté des pendants à charnière, on l'a vu, est une constante régionale. On dispose même, à Quarante et à Servian ( $\mathrm{n}^{\circ} 141$ et 177), d'une forme de pendant à crochet qui appartient plutôt au répertoire stylistique des pendants à charnière... Les pendants de Clermont-l'Hérault ne trouvent en fait que de rares parallèles méridionaux: une découverte ancienne d'Aix-enProvence (Ambard et al. 1972, 44, fig. 15), un pendant d'Albias (Tarn-et-Garonne), et plus à 
l'Ouest les objets de Guimps (Char.-Maritime) et du Langon en Vendée, ce dernier plus schématique (fig. 4). Comme l'un des exemplaires des fouilles récentes de Peyre-Plantade, le pendant du Langon provient d'un contexte du milieu du Ier s. de n. ère, illustrant l'apparition précoce de ce modèle. En Gaule, au moins, il ne semble cependant pas avoir concurrencé sérieusement les pendants à crochets, qui restent très clairement majoritaires.

Les agrafes, qui comptent parmi les objets de harnais les plus répandus, servaient à relier une lanière à une jonction métallique, anneau ou pièce plus complexe comme le montrent quelques trouvailles (fig. 5). C'est d'une période plus tardive (IIe-IIIe s.) que date une forme de pendant allongé et coulé, type classique, parfois associé à des appliques à anneau latéral (Fig. 6, 2), voire à des coquilles, comme sur la lanière du harnachement de Celles (Belgique) conservée avec cuir et appliques (Massart 2000, fig. 9).

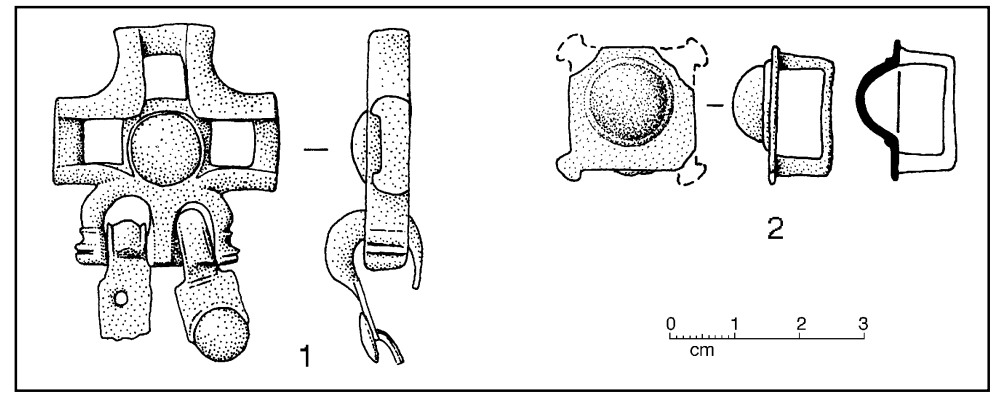

Fig. 5. Jonctions de harnais: 1, Vic-le-Fesq, «La Basane» (Gard) (prosp. M. Gilly, 1989); 2, Calvisson (Gard), coll. Rappaz, Nîmes. Ech. 2/3.

Quelques objets émaillés, notamment à Quarante, illustrent une certaine permanence du harnais décoré au IIIe s.; l'applique à bords concaves $\mathrm{n}^{\circ} 142$, notamment, est une forme caractéristique qui connaît de multiples variantes dans l'organisation interne des décors (fig. 6, 35); il semble d'ailleurs que cet exemplaire se rattache à une forme rare, à 6 côtés (par ex. Bonnin 1860, pl. XXXVII, $\mathrm{n}^{\circ} 25$ ). Boutons circulaires et appliques polygonales (ou ovales comme à Alleins dans les Bouches-du-Rhône: Feugère, Pillard 1999) pourraient avoir orné les harnais dont les mors comportent des appliques latérales en bronzes, illustrées ici à Espondeilhan et, dans leur forme la plus simple, à Ambrussum. Les deux séries sont en effet associées dans les trouvailles de harnais complets, comme celle de Feurs (Feugère 1983, $n^{\circ}$ 9-53). Une étude précise des objets de ce corpus permettrait peut-être de classer les objets des IIe et IIIe siècles, plus précisément que nous ne l'avons fait ici, mais elle reste à tenter, notamment à partir des ensembles funéraires de Belgique et de Pannonie.

Si bien des aspects obscurs subsistent pour le mobilier du Haut-Empire, que dire de l'Antiquité tardive? Il s'agit, pour une bonne part, d'objets très mal connus en Gaule du Sud: les travaux de référence se limitent généralement au Nord des Alpes ou à la Gaule septentrionale, et d'une manière générale les séries méditerranéennes demeurent moins étudiées. Certains travaux récents, comme celle de J. Aurrecoechea (2001), mettent pourtant en évidence l'originalité de plusieurs séries, ainsi que leur contribution à la problématique générale de ce mobilier et de son interprétation.

Le département de l'Hérault occupe pour cette période une position représentative de l'ensemble des départements français du littoral méditerranéen. On y rencontre à la fois des objets que leur isolement typologique permet de désigner comme des importations septentrionales, et des séries originales dont l'étude demanderait un plus large corpus. La boucle en cours 


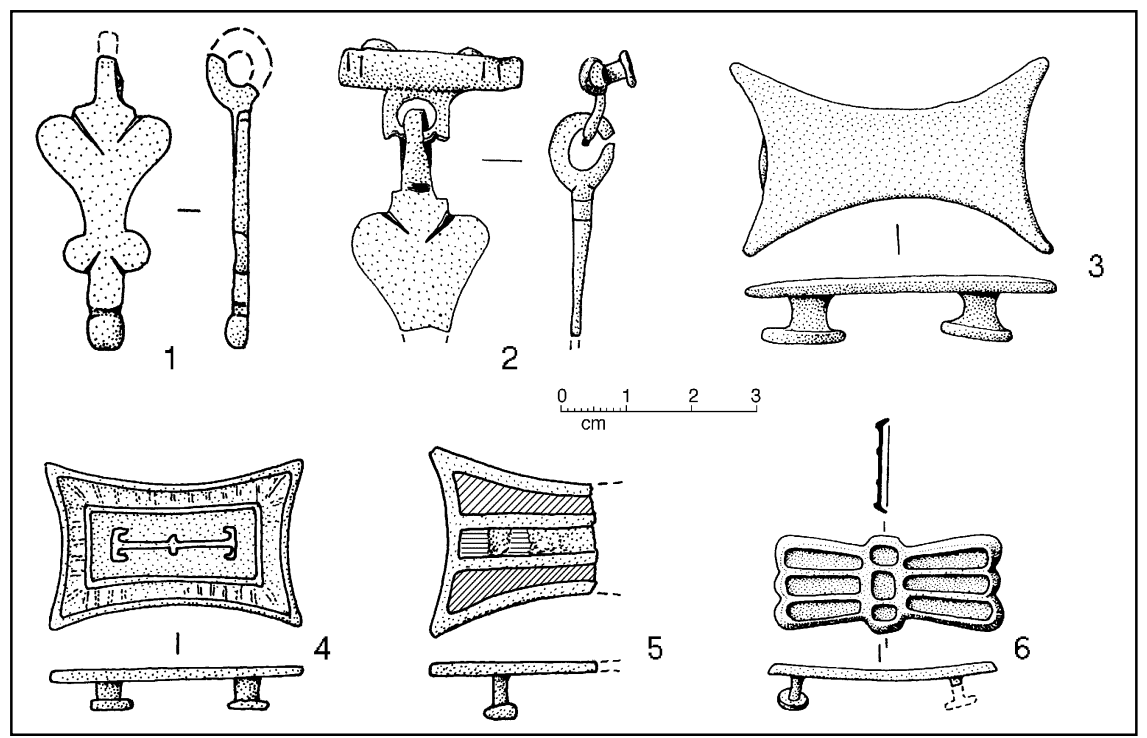

Fig. 6. Pendants coulés en forme de fleuron: 1 et 2, Bordeaux (Musée d'Aquitaine, inv. 90.34.1089 et 85.1.89). Appliques de harnais à bords concaves: 3 et 6, Feurs (Feugère 1983); 4, Le Langon, salle polyvalente (Vendée), fouilles E. Bernard; 5, Mâlain (Côte-d'Or). Ech. 2/3.

de fabrication de Neffiès, en particulier, prouve si besoin était que des objets de typologie «germanique» ont été produits sur les sites locaux, y compris à la campagne... Dans le domaine des boucles, des appliques et des ferrets de ceinture, des études élargies donneront sans doute un jour des résultats très novateurs pour cette période.

Limitons-nous ici à un seul exemple, celui du montage des ferrets: la plus grande partie de ces objets fait appel à une plaque fendue et rivetée sur l'extrémité de la lanière. Or un unique objet héraultais, pour le moment isolé en Gaule du Sud, comporte une plaque à rivets coulés, articulée sur le ferret proprement dit par une charnière (Roujan, $\mathrm{n}^{\circ} 148$ ). S'ils semblent très rares en Gaule, les ferrets articulés forment une part non négligeable du corpus de ces objets en Italie du Nord et en Pannonie. Il y a sans doute là une piste de recherche qui pourrait déboucher sur une appréciation plus fine des mouvements de personnes, révélés par ces parures caractéristiques.

\subsection{Interprétation générale}

Le corpus des militaria héraultais est très déséquilibré en faveur du Haut-Empire, et plus précisément du Ier s. de notre ère (fig. 7). En attribuant toutes les balles de frondes à la fin de la période républicaine (alors qu'une partie d'entre elles peut dater du début de notre ère), on ne dénombre pour cette période que 14 objets, qui représentent $6 \%$ du corpus complet. En dehors des casques en bronze, qui sont les objets les mieux étudiés à ce jour, l'objet le plus intéressant est un éperon d'Aumes $\left(n^{\circ} 16\right)$, peut-être datable de cette époque.

Le Haut-Empire est de très loin, on l'a dit, la période la mieux documenteé. Au sein de cette période, les armes ne représentent que $6,5 \%$ du mobilier, comme on pouvait s'y attendre: la pax romana ne laisse que peu de place à l'hypothèse de troupes stationnant dans la Province, à l'exception de passages ponctuels, surtout dans les agglomérations. Le reste du mo- 
bilier se répartit entre ceinture et harnachement, avec une proportion qui a sensiblement évolué vers ce dernier du fait de l'interprétation des boucles les plus simples, attribuées désormais au harnais. Si ce mobilier suscite toujours un débat passionné entre les tenants du «tout militaire» ou du "civil et militaire», les éléments issus du cingulum ne prêtent guère le flan à la critique: ce sont indubitablement des objets issus de fabricae militaires, retrouvés sur des sites civils. Comme les armes ci-dessus, ils indiquent l'existence de contacts continus entre les deux sphères. En additionnant ces deux catégories, ce sont $17,8 \%$ des objets de notre corpus qui, pour cette période, ont clairement une origine militaire: rien d'anecdotique, donc, mais au contraire un phénomène qu'il appartient de considérer dans un plus large contexte.

C'est probablement dans le domaine des objets liés au harnachement (plus de $80 \%$ du mobilier) que se trouve la réponse à la question posée par ces militaria. D'une manière générale, on sait que les recrutements de soldats effectués en Narbonnaise concernent essentiellement le plein Ier s., comme le montre le décompte de G. Forni, reproduit par Y. Le Bohec $(1989,87)$, sur les effectifs de recrues de cette province dont le nom nous est parvenu:

$$
\begin{aligned}
& \text { Auguste - Caligula: } 31 \text {; } \\
& \text { Claude - Néron: } 58 \text {; } \\
& \text { Flaviens - Trajan: } 34 \text {; } \\
& \text { Hadrien - fin du IIIe s.: } 6 \text {. }
\end{aligned}
$$

On est beaucoup moins renseigné sur le recrutement des auxiliaires, puisque seules deux unités (alae de Voconces) sont indiquées par G.L. Cheesman comme originaires de Narbonnaise (Le Bohec 1989, 99). Mais il ne s'agit là que d'unités levées en majorité dans une seule cité, une situation qui n'a pas dû être générale et se dissout rapidement du fait des remaniements: les nouvelles formations prennent alors des noms plus généraux, voire mixtes (alae Gallorum et Pannoniorum, par exemple), dans lesquelles l'origine des soldats n'apparaît plus de manière précise.

Il sera donc difficile de trouver des arguments textuels ou épigraphiques pour illustrer l'importance d'un recrutement de cavaliers dans la province; mais deux aspects méritent d'être soulignés. D'une part, comme on l'a dit souvent, un harnais se compose de plusieurs dizaines d'éléments métalliques, dont certains, comme les pendants à crochet, sont en mouvement perpétuel et sont donc susceptibles de se détacher facilement: cette caractéristique explique, en partie, la sur représentation du harnais par rapport aux autres catégories. D'autre part, dans l'armée du Ier siècle, et notamment au sein des corps d'auxiliaires, la cavalerie jouit d'un prestige particulier: en témoignent les aspects originaux et spectaculaires, maintes fois soulignés, de son organisation, de son équipement et de ses manifestations publiques (hippika gymnasia, stèles funéraires...).

Nous proposons donc une interprétation mixte des militaria héraultais, correspondant à une situation historique complexe:

1 il est indéniable que des soldats en activité ont séjourné dans la région, notamment dans les vici et dans les villes, où les militaria se distinguent par un faciès particulier. Naturellement, les pertes d'armes et d'équipement durant ces séjours actifs ne peuvent être que limitées, ce qui nous conduit à accorder une certaine importance aux rares documents de cette première catégorie.

2- les soldats recrutés en Narbonnaise, et en particulier dans l'actuel département de l'Hérault, sont en partie revenus au pays; ils ont pu y rapporter des parts «non sensibles» de leur équipement, témoins prestigieux de leur carrière militaire: cingulum, harnais... On sait d'autre part que des armes transformées en outils (scies de L'Hospitalet-duLarzac: Feugère 1993b, fig. 3, 2-3) ont pu être conservées par certains vétérans. L'abon- 

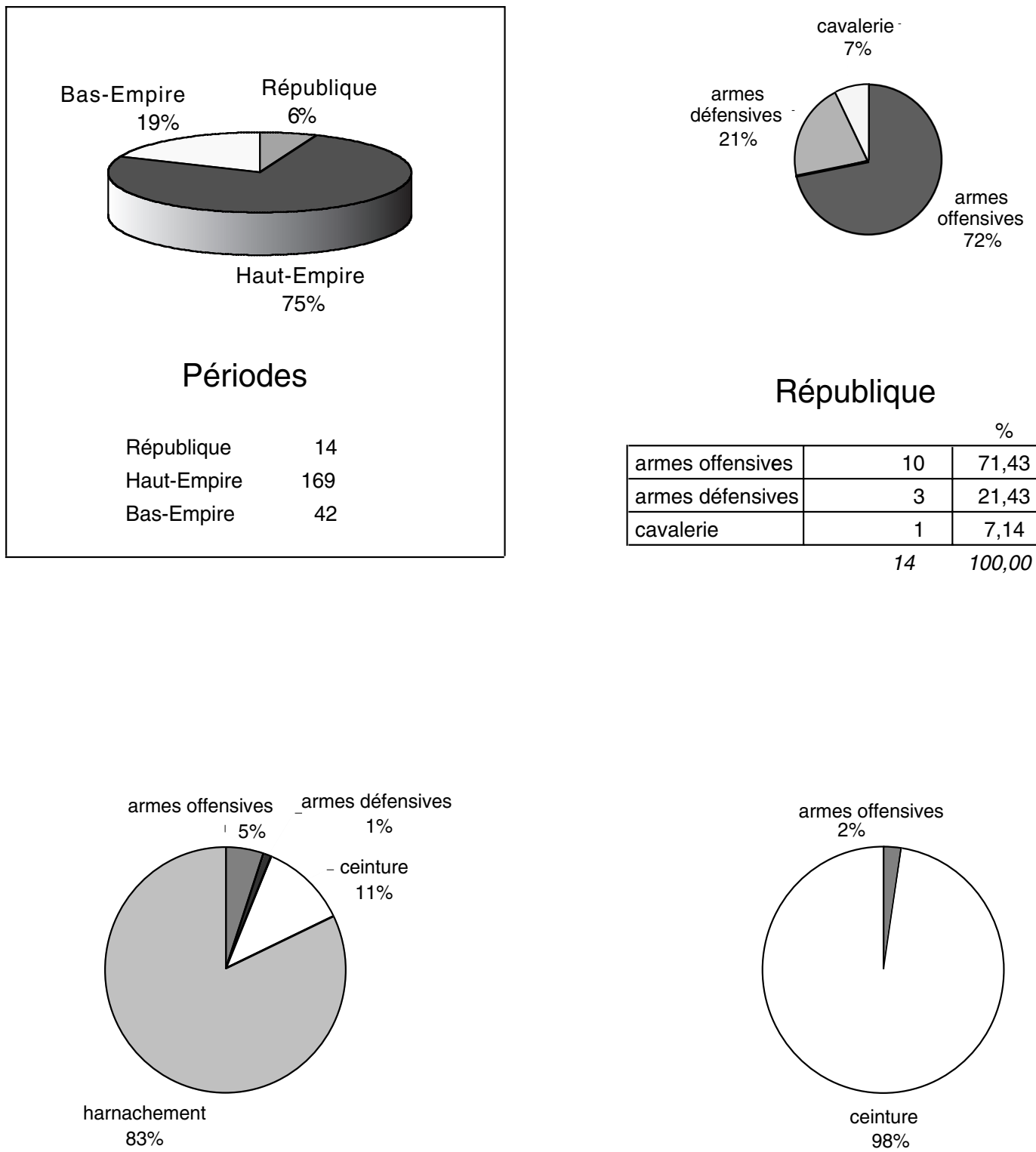

Haut-Empire

\begin{tabular}{|l|r|c|}
\hline armes offensives & 9 & 5,33 \\
\hline armes défensives & 2 & 1,18 \\
\hline ceinture & 19 & 11,24 \\
\hline harnachement & 139 & 82,25 \\
\hline \multicolumn{2}{l}{169} & 100,00 \\
\hline
\end{tabular}

Bas-Empire

\begin{tabular}{|l|r|c|}
\hline armes offensives & 1 & $\%$ \\
\hline ceinture & 41 & 97,38 \\
\hline \multicolumn{2}{|c|}{42} & 100,00 \\
\hline
\end{tabular}

Fig. 7. Repartition statistique des militaria de 1'Hérault: par periodes (en haut à gauche, puis attribution fonctionnelle pour chaque période. 
dance du mobilier militaire, même fragmenté ou détourné de sa fonction première, reflèterait ainsi l'importance des recrutements dans la région.

3- certains objets isolés peuvent enfin se retrouver sur des sites civils sans que l'on doive leur accorder une signification particulière: objets perdus et ramassés par hasard, etc... Cette interprétation doit néanmoins rester minoritaire dans la mesure où la grande dispersion des militaria sur les sites doit traduire une situation bien établie, et non une succession très improbable de cas exceptionnels.

Enfin, au Bas-Empire, le mobilier se réduit presque exclusivement à des éléments de ceinture. Derrière cette apparence d'uniforme se cachent sans doute des militaires, mais aussi tous les personnels officiels qui jouent un rôle dans l'administration régionale aux IVe et Ve siècles. Il s'agit donc d'une population mixte, parfaitement imbriquée dans le tissu social, et qui ne saurait être étudiée à partir de ce seul mobilier. L'examen des objets montre une situation complexe, avec des importations d'origines diverses, des productions locales et des styles ornementaux qui se démarquent progressivement d'un fond «germanique» largement assimilé au modèle romain en général. Deux pistes de recherche pourront être explorées pour progresser sur cet aspect de la question: une enquête morphologique et stylistique, pour replacer le mobilier héraultais dans un cadre plus large; et une démarche sociologique, à partir des sépultures à mobilier, pour apprécier la place des défunts pourvus de telles ceintures dans les populations locales.

\section{Catalogue (Fig. 8-22)}

Communes par ordre alphabétique, avec renvoi aux figures pour les objets illustrés².

\section{Abeilhan (Fig. 8)}

- Pech-Clavel

1. Boucle de cingulum, IVe-Ve s.; prosp. et don G. Cabrol; MPM, inv. 992.81.13; L. act. 40 $\mathrm{mm}$. Les boucles à simples volutes internes et deux charnons, presque absents des corpus de Böhme (1974, pl. 97, 1) et Sommer (1984) appartiennent à des plaques-boucles articulées de type hispanique: on les rencontre notamment sur les plaques-boucles de type «Simancas», de la fin du IVe et du Ve s. de notre ère.

2. Ferret de ceinture, IVe-Ve s.; prosp. et don G. Cabrol; MPM, inv. 997.30.1; L. act. 40,5 $\mathrm{mm}$. Sommet d'un pendant foliacé à décor excisé; deux rivets de bronze en place pour la fixation à l'extrémité de la ceinture. La suspension, trapézoïdale, est chanfreinée et ornée de doubles lignes de ponctuations estampées. Le décor excisé, limité par une ligne de ponctuations entre deux cannelures, comportait au sommet une «fleur de lys». La présence d'un ergot sur l'angle inférieur gauche laisse supposer la présence d'un motif zoomorphe autour de la pointe (forme B, type C, variante 1 de Sommer 1984; cf. par ex. Böhme 1974, pl. 100, 5).

3. Ferret de ceinture, IVe-Ve s.; prosp. et don G. Cabrol; MPM, inv. 999.26.1; L. act. 65 $\mathrm{mm}$. La forme (amphore à panse effilée) est classique, mais le décor d'ocelles semble particulièrement abondant sur les ferrets du département: il s'agit probablement d'un style régional.

\footnotetext{
2 Les objets oubliés ou trouvés après la clôture du catalogue ont été insérés en numéros bis, sans illustration. 3 MPM: Maison du Patrimoine (Musée municipal) de Montagnac (Hérault).
} 
- Ruisseau de l'Etang (habitat?)

4. Applique de ceinture, IV-Ve s.; prosp. et coll. G. Cabrol (copie à la MPM); L. 32,5 mm. Elle figure, en relief plat, un cheval tourné vers la gauche; au revers, deux rivets de fixation. Le seul parallèle régional est fourni par une applique reprenant le motif central de la plaque-boucle sur le ceinturon d'Argeliers, datable du Ve s. (en dernier lieu: Aurrecoechea 2001, fig. 80).

Agde (Fig. 8)

- ville

5. Pendant de phalère, type à crochet; fouilles R. Aris, Musée Agathois, inv. 93/78; larg. act. 50,5 mm (restit. env. $62 \mathrm{~mm}$ ), ht. act. $57 \mathrm{~mm}$. Lest biconique.

\section{Alignan-du-Vent (Fig. 8)}

\section{- Le Valat}

6. Ferret de ceinture, contexte fin IVe au courant du VIe s.; fouilles et don S. Mauné, h.s.; MPM, inv. 998.68.7; L. act. $45 \mathrm{~mm}$. Pendant foliacé, en forme d'amphore, en tôle mince à l'exception du bouton terminal (forme B de Sommer 1984), et dont le décor — qui ne concerne qu'une face, l'autre restant lisse - associe un motif central de cercles concentriques à une bordure incisée de doubles traits «a tremolo» (décor proche, mais sur une forme différente, à Vermand: Böhme 1974, pl. 140, 4; Wizernes: ibid., pl. 147, 8).

7. Applique de ceinture, contexte fin IVe au courant du VIe s.; prosp. et don G. Cabrol; MPM, inv. 991.15.1; L. 37,5 mm. Plaque triangulaire coulée, percée de deux trous de fixation; l'une des faces porte un décor excisé composé d'une large frise de grecques encadrant un motif triangulaire. Des appliques de formes variées (triangulaire à base étroite, comme ici, ou plus large, rectangulaire ou losangique) se rencontrent sur les larges ceintures à décor excisé de type Böhme $\mathrm{A}$, qui en comportent généralement cinq. Les appliques triangulaires sont très rares en Gaule du Sud, où nous ne pouvons citer actuellement que deux autres appliques à base large et décor excisé: l'une dans la grotte de SallèlesCabardès (Aude) (Passelac 1968, pl. II), l'autre près de Puylaurens (Tarn) (rens. † J.-F. Salinier).

8. Plaque de ceinture, contexte fin IVe au courant du VIe s.; prosp. et don G. Cabrol; MPM, inv. 996.41.1; L. act. $74 \mathrm{~mm}$, larg. act. $57 \mathrm{~mm}$. Boucle à décor excisé, évidée pour la boucle et son ardillon, qui étaient placés au centre. La garniture complète comportait 3 éléments, deux contreplaques à pointe triangulaire venant compléter la plaque-boucle rigide (Böhme 1974, type B); la pointe triangulaire de la plaque-boucle d'Alignan est une variante de la forme rectangulaire canonique (v. par ex. Tournai: Böhme 1974, pl. 109, 3).

- Grauzan

V. ci-dessous $n^{\circ} 33$.

\section{Aspiran (Fig. 8)}

- Gissos

10. Pendant de harnais, Ht-Emp.; prosp. et don H. Leyris; MPM, inv. 993.70.1; L. act. 23 $\mathrm{mm}$. De très petits pendants de ce type, à triple perforation, s'observent sur des extrémités de lanière attribuées au harnachement (Vindonissa: Unz, Deschler-Erb 1997, pl. 63, 1797-1798); $c f$. ici Lattes, Mèze et Sauvian. 


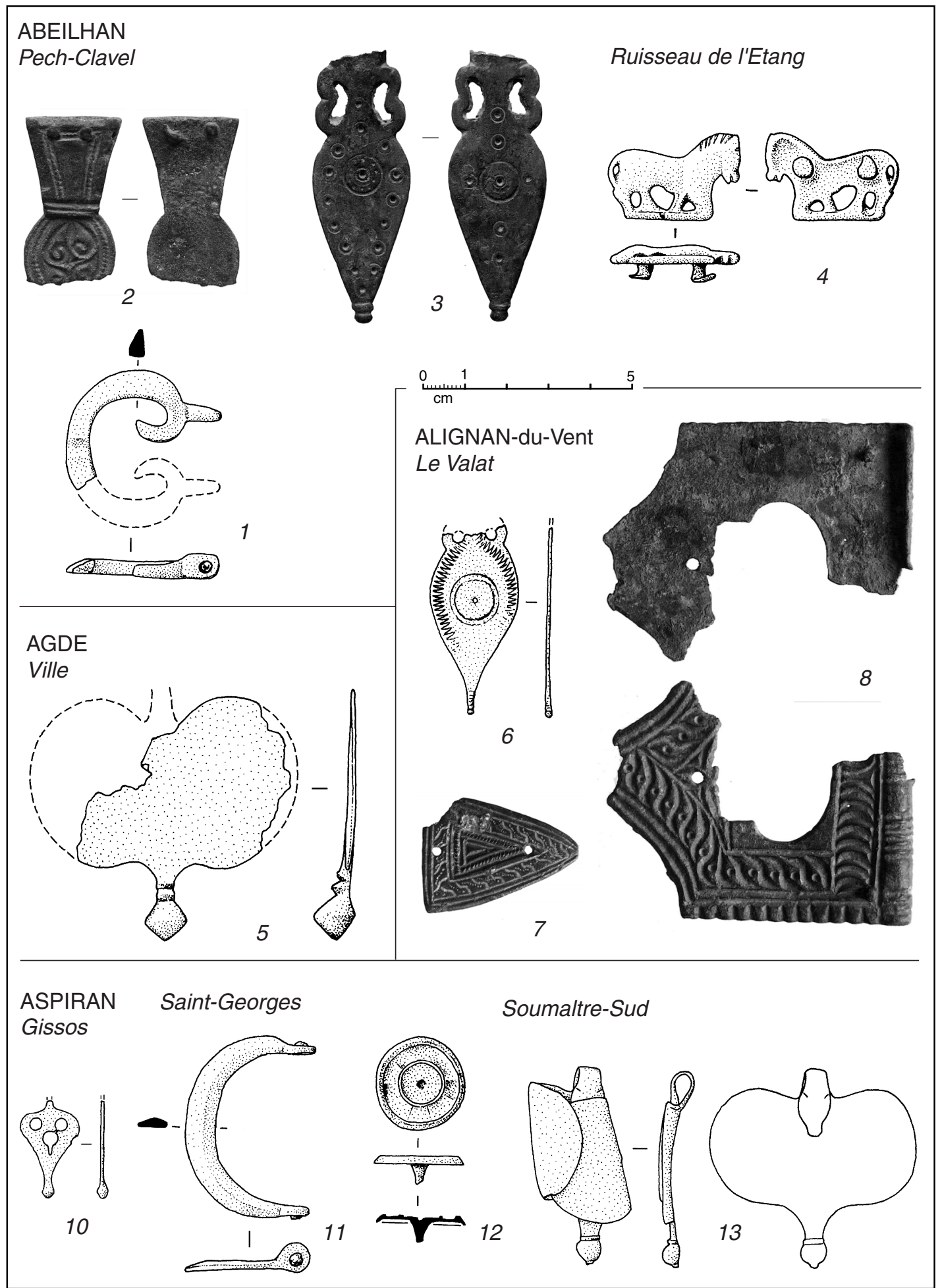

Fig. 8. Militaria du département de l'Hérault (v. le catalogue). Ech. 2/3. 


\section{- St-Georges}

11. Boucle de harnais, Ier-IIe s.; prosp. et don G. Cabrol; MPM, inv. 990.76.1; L. 43,5 mm. Forme classique pour ces boucles légères, longtemps attribuées au cingulum, mais que l'on préfèrera désormais classer dans le harnais (v. fig. 2).

12. Bouton émaillé; prosp. et don G. Cabrol; MPM, inv. 995.83.1; diam. 21,5 mm. L'empreinte des émaux disparus permet de restituer un décor comportant 5 inclusions de verre noyées dans l'émail; cette technique décorative n'apparaît pas avant le IIe s.

- Soumaltre-Sud

13. Pendant de harnais à crochet; fouilles Afan, A75, R. Thernot, 1999, us 8007; ht. $47 \mathrm{~mm}$. Type classique du pendant en tôle, lest en forme d'oignon: le modèle plus répandu en Languedoc.

\section{Aumes (Fig. 9)}

- Oppidum

14. Bord de casque italique, IIe-Ier s. av. J.-C.; prosp. et don J.-C. Rouanet (Saint-Auby, parc. 188/192); MPM, inv. 2001.77.4; L. act. $27 \mathrm{~mm}$. Le profil épais $(5 \mathrm{~mm})$, mouluré et guilloché, est caractéristique des divers modèles de casques italiques utilisés en Gaule méridionale aux IIe et Ier s. av. n. ère. Il n'est malheureusement pas possible d'attribuer ce fragment à l'un des deux modèles coulés, le type à bouton sommital (-IIe s.) ou le type Coolus (-Ier s.). Mais avec les fragments récemment découverts à Nîmes et à Marseillan, cette découverte semble indiquer une utilisation des casques en bronzes plus répandue que ce que l'on pouvait supposer jusqu'ici à partir des seuls objets complets.

15. Balle de fronde en plomb, -Ier/+Ier s.; prosp. (1988, parc. 304) et don G. Cabrol; MPM, inv. 993.54.1; L. $34 \mathrm{~mm}$. Les deux extrémités sont aplaties comme si elles avaient été martelées.

16. Éperon, prosp. et don A. Rougerie; MPM; L. act. $71 \mathrm{~mm}$. La branche conservée, de section filiforme et simplement ornée à chaque extrémité de fines incisions transversales, se termine par une plaque de fixation triangulaire simplement percée; l'aiguillon était en fer. Ce type d'éperon, encore mal connu, est attesté sur les camps autour de Numance (Schulten 1927, pl. 35: 19 and 22; Egg, Pare 1995, 232, nº 3, Bronzesporn, Farbtaf. 31). On peut également citer un éperon comparable de l'habitat de Langa de Duero, Soria (Stary 1994, 44, no 6 et B. Taracena Aguirre, Mem. Junt. Sup. Exc. Ant. 119, 1931, pl. 34 right; rens. D. Božič).

17. Bouton à anneau; prosp. et don G. Cabrol; MPM, inv. 996.7.1; L. 31 mm. Type à plaque rectangulaire moulurée transversalement sur la face supérieure, caractéristique de l'époque augustéenne:

18. Bouterolle de glaive, Ier-IIe s.; prosp. et don A. Rougerie; MPM, inv. 988.1.17; Feugère 1993a, 146. Bouterolle coulée d'une seule pièce, comportant à la base un bouton arrondi, séparé par deux moulures de l'étui enserrant la pointe du fourreau. Sur les deux faces, ajours séparés par un motif en S. Ce type de bouterolle appartient aux glaives romains de type Pompei: sur ce modèle, en effet, la bouterolle n'est plus solidaire des renforts latéraux en gouttière comme elle l'était sur le type Mainz.

19. Pendant de harnais, type à crochet; prosp. et coll. A. Rougerie; larg. 48,5 , ht. act. 40 $\mathrm{mm}$. Aucun décor sur la face externe; le crochet, qui était sans doute en forme de tête d'oiseau, a disparu.

20. Crochet de pendant de phalère; prosp. et coll. A. Rougerie; L. act. $24 \mathrm{~mm}$. Crochet façonné en forme de tête de canidé, un motif nettement moins répandu que la tête d'oiseau aquatique. 


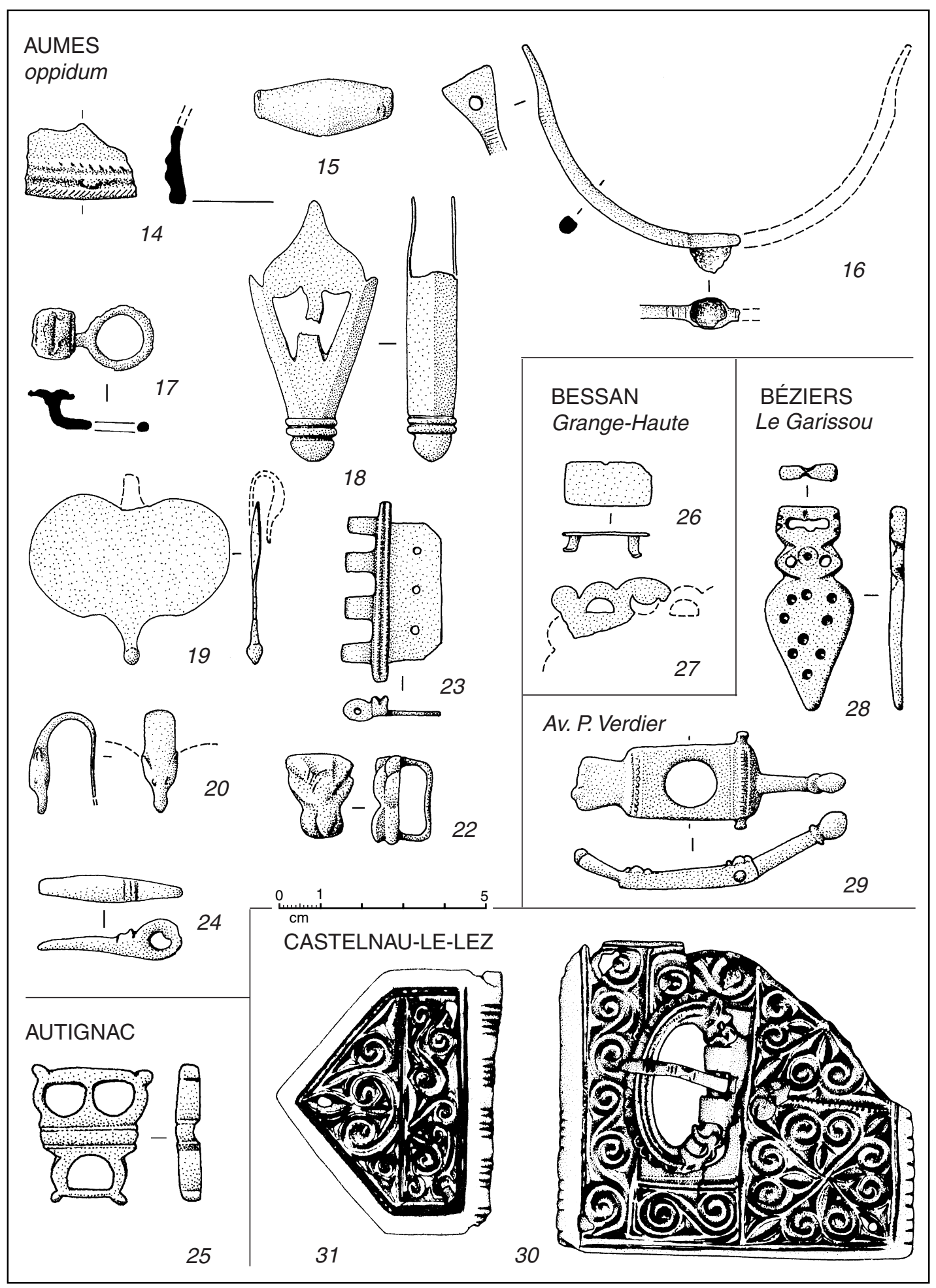

Fig. 9. Militaria du département de l'Hérault (v. le catalogue). Ech. 2/3. 
21. Passant de harnais; prosp. et coll. A. Rougerie; L. 13,5 mm. Il semble s'agir d'une version simplifiée (sans les boutons terminaux) de la forme plus classique attestée localement à Clermont-l'Hérault et à Pouzolles.

22. Passant de harnais figuré; prosp. et coll. A. Rougerie; L. 21,5 mm. Passant rectangulaire dont la face externe figure des parties génitales masculines.

23. Plaque de cingulum, Ier s.; prosp. et don J.-Cl. Rouanet; MPM, inv. 2001.1.29; L. 24, larg. $44 \mathrm{~mm}$. Boucle articulée (4 charnons, les deux du centre enserrant l'ardillon) pourvue d'une simple plaque à 3 rivets de fixation. Ces boucles très simples appartiennent au cingulum du début de l'Empire: elles sont bien représentées sur le limes germanique et dans le Sud de la Bretagne, ce qui plaide pour une datation vers le milieu ou la deuxième moitié du Ier s (Böhme 1978, carte fig. 74; cf. à Augst: Deschler-Erb 1999, nº 369-370, ce dernier exemplaire daté c. 30-70 ap. J.-C.). En Languedoc-Roussillon, une boucle de ce type est connue à Sigean, Aude (MPM, inv. 2001.63.1).

24. Ardillon de boucle en bronze; prosp. et coll. A. Rougerie; L. $35 \mathrm{~mm}$. Objet coulé, orné de deux côtes transversales avant la charnière.

Autignac (Fig. 9)

- lieu-dit?

25. Jonction de harnais; prosp. et coll. G. Cabrol; L. $24 \mathrm{~mm}$. Robuste pièce de jonction pour trois agrafes. On peut la comparer à diverses pièces attestées en contexte militaire à partir de l'époque augustéenne, comme par exemple à Dangstetten (Fingerlin 1986, 193,2).

\section{Bessan (Fig. 9)}

- Grange-Haute (établissement gallo-romain)

26. Applique rectangulaire; prosp. et don J.-Cl. Rouanet; MPM, inv. 2001.75.12; L. act. $21,5 \mathrm{~mm}$. Ce type de garniture, très simple, est attesté parmi les productions d'objets de harnais d'Alésia (Rabeisen 1990, fig. 6, 12).

27. Bordure d'applique de harnais; prosp. et don J.-Cl. Rouanet; MPM, inv. 2001.75.14; L. act. $29 \mathrm{~mm}$. L'alternance des ajours est caractéristique de ces appliques de harnais de mieux en mieux connues depuis quelques années (Abauzit 2000; Božic 2001).

\section{Béziers (Fig. 9)}

- Le Garissou (villa gallo-romaine)

28. Ferret de ceinture, IVe s.; coll. B. Lenoir; Feugère 1993a, 253,18; L. 49 mm. Si la forme générale et le décor ocellé sont conformes à ce que l'on connaît des ferrets tardoromains dans la région, voire au-delà, le type de fixation est original.

- Av. Pierre Verdier (tombe gallo-romaine)

29. Amulette phallique; travaux 1935: une ou plusieurs tombes, dont le mobilier n'a pu être récupéré que regroupé; Musée du Biterrois; Lapeyre 1987-88, 19, pl. VI, nº 14; L. 67 mm. Type classique de cette amulette, dont la fonction primaire demeure incertaine (quelques exemplaires ont été retrouvés comme ici en milieu funéraire); v. localement la découverte de Fontès.

\section{Castelnau-le-Lez (Fig. 9)}

- lieu-dit inconnu (inhumation?)

30. Plaque-boucle, vers 400 ap. J.-C.; Musée de Montpellier; James 1977, vol. 2, pl. 187; Feugère 1993b, 147, fig. 15; L. 85 mm. V. ci-dessous. 
31. Contre-plaque triangulaire, vers 400 ap. J.-C.; James 1977, vol. 2, pl. 187; Feugère 1993b, 147, fig. 15; L. $53 \mathrm{~mm}$. Objets découverts en 1857, dans des circonstances inconnues; mais l'association d'une plaque-boucle et d'une contreplaque indique très probablement une découverte funéraire. Les deux éléments conservés appartiennent à une garniture de type Böhme B, en 3 parties.

\section{Caux (Fig. 10)}

- Les Crouzals (établissement gallo-romain)

32. Phalère de harnais, I-IIe s.; prosp. et don G. Gauthier; MPM, inv. 990.70.1; Depeyrot et al. 1986, fig. 8A, 1; diam. $54 \mathrm{~mm}$. Phalère épaisse, coulée, creuse à l'arrière, dont la fixation devait être assurée par une ou deux bélières: traces d'arrachement au revers; possible ornement central rapporté. Décor de 4 palmettes stylisées (et points alignés?), sans doute niellées à l'origine.

- Grauzan (établissement gallo-romain) ${ }^{4}$

33. Bouton à anneau; prosp. et coll. G. Cabrol; copie à la MPM; L. $34 \mathrm{~mm}$. La tête du bouton représente une tête de Jupiter Ammon. Ce traitement est tout à fait exceptionnel pour ce type d'objets, où l'on observe en général, du moins en Gaule, qu'un bouton lisse ou très sommairement orné de cercles gravés ou niellés, comme sur l'exemplaire ci-dessous de Neffiès. Un bouton identique a néanmoins été trouvé sur le camp légionnaire de Vindonissa (Unz, Deschler-Erb 1997, pl. 71, no 2099). Jupiter Ammon apparaît sur toute une série de figurations romaines que l'on fait généralement remonter à un relief du portique du forum d'Auguste, à Rome ${ }^{5}$; le motif se diffuse notamment en contexte militaire, comme l'illustrent les phalères de Lauersfort, les statues cuirassées, de nombreux médaillons et autres appliques en, bronze (par ex.: Boschung 2000, fig. 14); on notera enfin sa présence sur une phalère de Neffiès (infra).

\section{Clermont-I'Hérault (Fig. 10)}

- Peyre-Plantade (agglomération gauloise et gallo-romaine)

34. Boucle (de lorica?); us $6305^{6}$ (remblai, vers 75 / 150 ap. J.-C.); L. 22,5mm. Boucle en $\mathrm{D}$, de petite taille, ayant conservé son axe en fer et son ardillon en bronze; elle reprend le modèle, très simple, des boucles longtemps attribuées au cingulum et maintenant considérées comme appartenant au harnais. De telles boucles, prolongées d'une plaque de tôle repliée, sont souvent interprétées comme des charnières de jonction pour les cuirasses segmentées; il est cependant difficile de prétendre qu'une plaque de tôle aurait pu disparaître, sur notre exemplaire, sans endommager l'axe en fer. On rencontre enfin de petites boucles de même taille et de même facture dans les ensembles livrant des glaives complets avec leur fourreau: elles peuvent alors avoir servi à la suspension du fourreau, comme sur l'exemplaire retrouvé dans la couche d'incendie d'une maison de Délos (Bull. Corr. Hel. 1987)).

35. Anneau de cuirasse segmentée de type Newstead (Hadrien au mil. IIIe s.); prosp. et don M. Feugère et J.-Cl. Rouanet; MPM, inv. 2001.44.10; L. 21 mm. Il s'agit là de l'une des très rares attestations de ce type de cuirasse en Gaule du Sud. Le type est en revanche bien connu dans les contextes militaires du IIe s.: outre le site éponyme (Bishop 1999), par exemple à Aldborough (Bishop 1996, n 417-419) ou Eining (Jütting 1995, $\left.n^{\circ} 76-94\right)$.

\footnotetext{
4 Placé par erreur sur la commune de Caux, ce site appartient en fait à la commune d'Alignan-du-Vent.

5 V. Kockel in: Kaiser Augustus und die verlorene Republik, Berlin 1988, 191 sqq.

6 Us: unité stratigraphique: chaque numéro renvoie à l'inventaire de la fouille récente.
} 
36. Pendant de phalère; us 6420 (sédimentation de rue, vers 25 av / 50 ap. J.-C.); L. $63 \mathrm{~mm}$. Pendant de harnais dont la suspension était assurée par une charnière sommitale: ce type de pendant vient compléter une phalère équipée au revers de la partie femelle de cette articulation. Le motif est classique, mais connaît de très nombreuses variantes (Bishop 1988, fig. 43). L'élément principal, qui peut être traité dans un style végétal, retombe sur les côtés en deux motifs redressés vers le haut, dont la base est lestée de glands. La partie centrale est nouée (bague transversale) et forme un feston. À la différence de plusieurs exemplaires qui portent un décor de pampres gravés, ce pendant est simplement étamé. Le triangle en relief placé au centre semble peu courant sur les objets de même forme. Apparus dès l'époque augustéenne, les pendants à charnière ne se multiplient que dans la deuxième moitié du Ier s. (Vindonissa, avant 101 ap. J.-C.: Unz, Deschler-Erb 1997, pl. 51-52, $\mathrm{n}^{\mathrm{o}}$ 1380-1406). En Gaule méridionale, ce type est considérablement moins commun que le pendant de harnais à crochet de la première moitié du Ier $\mathrm{s}$ (fig. 4; v. néanmoins ci-dessous).

37. Pendant de phalère; us 3227 (comblement de fosse, vers 375 / 500 ap. J.-C.); L. act. $51 \mathrm{~mm}$. V. ci-dessus (cet objet est intrusif dans le contexte tardif où il a été retrouvé).

- Rhônel (quartier sud de l'agglomération de Peyre-Plantade)

38. Applique de harnais, Ier. s.; fouilles S. Barbey, 1999; surface; L. $87 \mathrm{~mm}$. Ce type d'applique, caractérisé par deux attaches latérales en lunules encadrant une pièce centrale rectangulaire, ne semble pas très répandu mais connaît une vaste diffusion dans les provinces occidentales; les parallèles les plus proches sont fournis, à l'Ouest, par une découverte inédite de Carcassonne ou environs (plaque centrale avec décor niellé; rens. E. Laffitte), et à l'Est par un fragment de Vaugrenier (Alpes-Mar.: Olivier, Rogers 1978, fig. 51). Autres parallèles: Deschler-Erb 1999a.

39. Ardillon de boucle en bronze, Ier. s.; fouilles S. Barbey, 1999, u.s. 5035; L. 42 mm. Cet ardillon imposant a dû appartenir à une grosse boucle en bronze (harnais ou cingulum).

40. Passant de harnais; fouilles S. Barbey, 1999, us 2000; L. 19,5 mm. Passant rectangulaire en baguette, attesté localement à Pouzolles $\left(\mathrm{n}^{\circ} 133\right)$ et d'une manière plus générale sur les sites militaires du Ier s. (Saintes: Bouchette et al. 1998, no 34-44; Vindonissa: Unz, Deschler-Erb 1997, pl. 75, nº 2329-2332).

\section{Corneilhan (Fig. 10)}

\section{- La Crouzette (ou Le Mouillou)}

41. Bouton à anneau, I-IIe s.; prosp. et don G. Fédière; MPM, inv. 993.12.1; L. 33 mm. Objets identiques à Lattes (ci-dessous, $\mathrm{n}^{\mathrm{o}}$ 57) ainsi, par exemple, qu'à Lautrec (Tarn), à Feurs (Loire) (Feugère 1983, fig. 9) et au Musée d'Aquitaine, à Bordeaux.

\section{Cruzy (Fig. 10)}

\section{- La Gare}

42. Pendant foliacé, Ier s.; Musée de Cruzy (rens. P. Abauzit); L. 94 mm. Rivet central avec restes d'un rivet de bronze, appartenant sans doute à un ornement disparu.

\section{Espondeilhan (Fig. 11)}

- lieu-dit inconnu

43. Applique latérale de mors, IIIe s.; coll. part.; L. 93 mm, larg. 57 mm. Feugère 1996a. Applique de forme circulaire, ornée d'un motif d'ajours formé de croix imbriquées dans un carré. Deux appendices, l'un circulaire, l'autre rectangulaire, sont diamétralement opposés. La tendance à un décor ajouré foisonnant s'observe généralement sur les appli- 


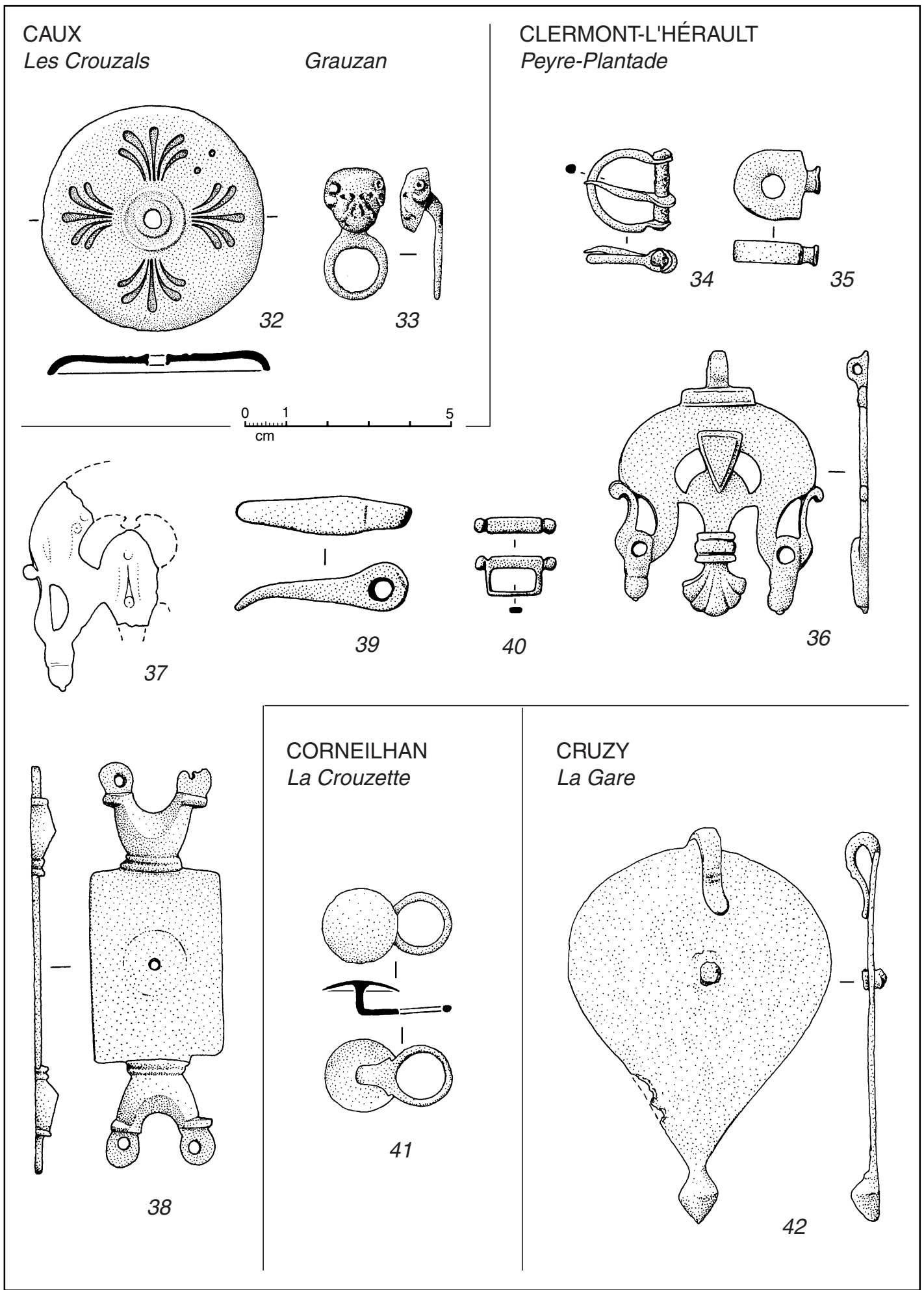

Fig. 10. Militaria du département de l'Hérault (v. le catalogue). Ech. 2/3. 
ques latérales de mors du IIIe s., période à laquelle ces pièces se multiplient, si l'on en croit des inventaires provinciaux comme celui de Chr. Boube sur le Maroc (Boube 1980). C'est donc à cette époque que l'on attribuera cet objet dont le contexte, et même la provenance précise, demeurent inconnus.

\section{Fabrègues (Fig. 11)}

\section{- La Roque}

44. Casque étrusco-italique à bouton sommital, IIe s. av. n. ère; Musée de Montpellier; Louis 1953. Cette forme désormais mieux connue (Schaaff 1988; Waurick 1990; Feugère 1994; Quesada 1997) ne semble guère avoir été utilisée en gaule du Sud après la fin du IIe s. av. n. ère, à quelques exceptions près; comme l'a noté U. Schaaff, le décor de vagues incisées, commun à plusieurs de ces casques découverts en France, pourrait indiquer un approvisionnement dans un atelier commun.

\section{Florensac (Fig. 11)}

\section{- La Gardie}

45. Crochet de pendant de phalère; prosp. et don M. Feugère, P. Gouyon; MPM, inv. 2001.36.5; L. act. $43 \mathrm{~mm}$. Ce crochet en forme de tête de canidé est remarquable par sa grande taille. Malgré le traitement réaliste de cet exemplaire, il n'est pas toujours facile de répartir les objets isolés entre simpula tardo-républicains et pendants de phalère, interprétation retenue ici.

\section{- San Peyre}

46. Pendant de harnais, type à crochet; prosp. et coll. J. Moles, Florensac; Depeyrot et al. 1986, fig. 11b; L. act. $57 \mathrm{~mm}$. Type habituel des pendants précoces à lest en forme d'oignon; premiere moitié du Ier s.

\section{Fontès (Fig. 11)}

\section{- Les Pradesses}

47. Agrafe de harnais; prosp. et coll. P. Gauthier, Sommières; Depeyrot et al. 1986, fig. 20, 12; L. act. $49 \mathrm{~mm}$. Type classique d'agrafe de harnais, permettant l'articulation d'une lanière sur un anneau.

48. Suspension de pendant de harnais; prosp. et coll. P. Gauthier, Sommières; Depeyrot et al. 1986, fig. 20,13; L. act. $20 \mathrm{~mm}$. Plaque à deux perforations assurant la fixation d'un pendant à crochet sur la lanière du harnais (première moitié du Ier s.; Oberstimm: Böhme 1978, B168).

49. Applique de harnais (?), Ht-Emp.; prosp. et don G. Jacquet; MPM, inv. 989.7.1; L. act. $36 \mathrm{~mm}$. Objet coulé, dont ni la forme ni la section épaisse, avec arête centrale, ne correspondent à la typologie des pendants de harnais. On ne peut guère hésiter, cependant, à attribuer ce décor à la catégorie du harnachement.

50. Pendant ou amulette phallique; prosp. et coll. P. Gauthier, Sommières; Depeyrot et al. 1986, fig. 20, 5; L. act. $28 \mathrm{~mm}$. Ce type relativement courant, dont les extrémités reproduisent celles des pendants de harnais phalliques (Bishop 1988, fig. 48-49, type 10) demeure énigmatique quant à sa fonction. Les seuls ensembles fonctionnels où il a pu être observé sont des colliers d'amulettes déposés dans des tombes du Haut-Empire (Baralle: Hosdez, Jacques 1989, 102, tombe J87 - J64, d'époque flavienne): son éventuel usage 


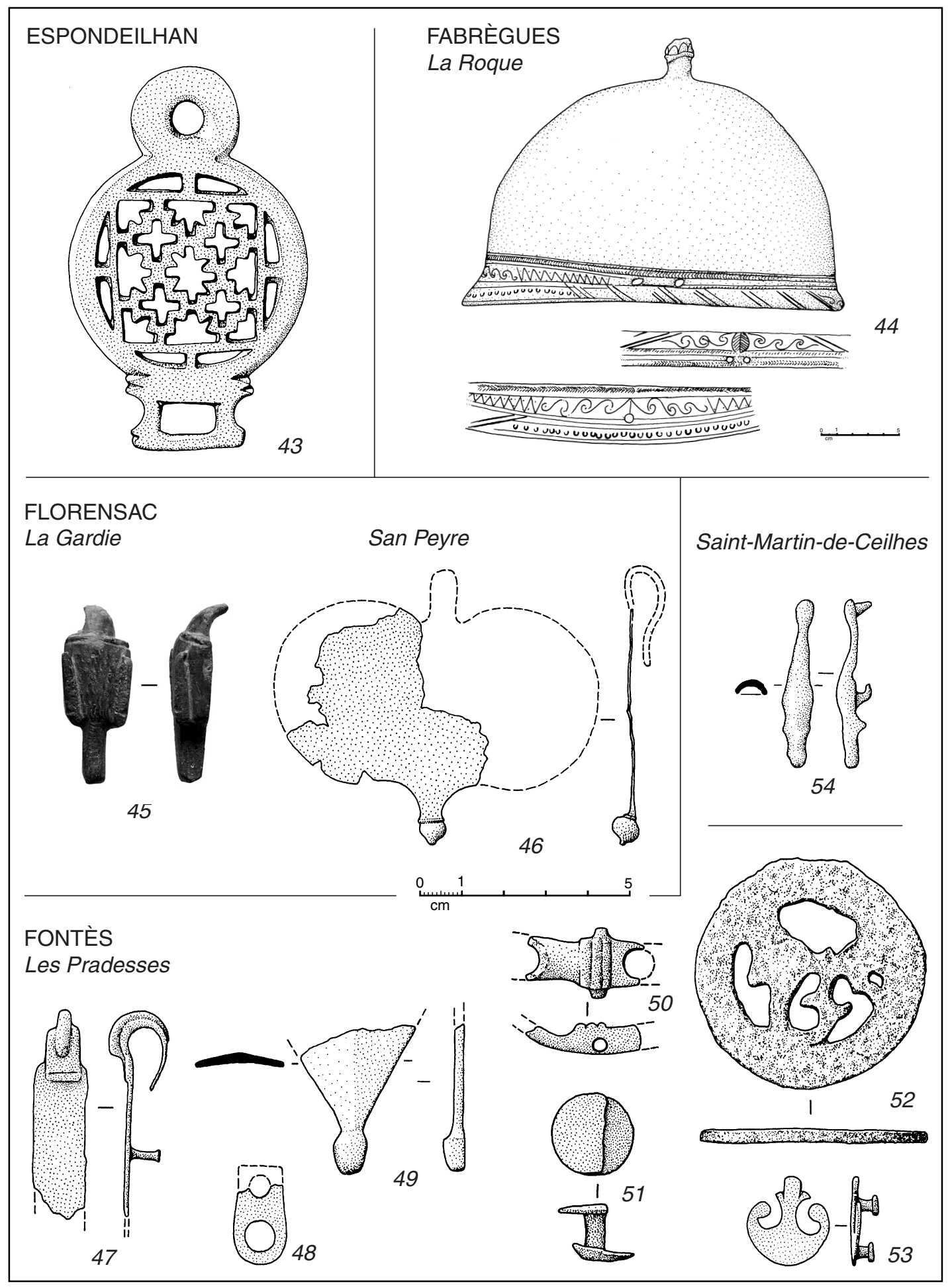

Fig. 11. Militaria du département de l'Hérault (v. le catalogue). Ech. 2/3. 
militaire demeure donc incertain. Un atelier a été signalé dans les Ardennes, à ChâteauPorcien (Maquart 1936).

51. Bouton; prosp. et coll. P. Gauthier, Sommières; Depeyrot et al. 1986, fig. 20, 10; ép. 8,5, diam. $19 \mathrm{~mm}$. Utilisé notamment dans les baudriers de spatha du IIIe s. (v. la tombe de Lyon: Feugère 1993a, 149), ce type de bouton trouve aussi de nombreuses applications dans les réglages de lanières de harnais.

52. Applique de harnais ou de mors?; prosp. et coll. P. Gauthier, Sommières; Depeyrot et al. 1986, fig. 20, 14; diam. $54 \mathrm{~mm}$. La forte épaisseur de ce disque ajouré est surprenante pour un décor d'applique; l'objet est d'autre part dépourvu de tout système de fixation. Malgré l'absence de parallèle, il pourrait s'agir d'une applique latérale de mors du IIIe siècle.

53. Applique de harnais; prosp. et coll. P. Gauthier, Sommières; Depeyrot et al. 1986, fig. 20,11; L. $22 \mathrm{~mm}$. Applique de forme classique (pelte) équipée de deux rivets de fixation au revers. Ce type d'applique, attesté sur les harnais complets du IIIe s. (Barcoczi 1946/48, fig. 4; Feugère 1983, 51-56), se retrouve dans certaines inhumations du IVe-Ve s., où des objets récupérés ont pu servir de décor de ceinture.

- St-Martin-de-Ceilhes.

54. Pendant de lanière, Ht-Emp.; prosp. et coll. P. Gauthier, Sommières; Depeyrot et al. 1986, fig. 21a; L. $40 \mathrm{~mm}$. Objet courant que l'on préfèrera attribuer au harnachement plutôt qu'à l'équipement personnel (ce qu'illustrent notamment les objets de la «fosse à char» de Saintes: Bouchette et al. 1998, fig. 45, 29-33).

\section{Frontignan (Fig. 12)}

\section{- Chemin des Romains}

55. Boucle de ceinture de type Teba; tombe 2, IVe s.; Musée de Sète; Pellecuer 1986, pl. 1, 5 et pl. 3, 1; Feugère 1993a, 253,4; 1996, 277, 4. Type de boucle caractérisé par une double fenêtre, la barre ajustée sur la lanière de cuir étant distincte de la boucle proprement dite. Bien attesté en Espagne, ce type est plus fréquent en Gaule méridionale qu'on pouvait le croire il y a quelques années: des boucles de Frontignan, de Minerve et du Musée de Montpellier illustrées ici, on rapprochera une découverte de Bages, Aude, inédite (MPM, inv. 2000.3.1) et la boucle de la nécropole de la Brèche à Laudun, Gard (tombe 19; Feugère et al. 1987; 1993b, fig. 14, 5).

56. Applique de ceinture; tombe 5, IVe s.; Pellecuer 1986, pl. 1, 13 et pl. 3, 4; Feugère 1993a, 253, 13; L. $46 \mathrm{~mm}$. Type classique de l'applique «en forme d'hélice», assez peu répandue en Gaule du Sud (v. ci-dessous Servian, n 174).

\section{Lattes (Fig. 12)}

- St-Sauveur (agglomération et port protohistoriques, puis gallo-romains)

56 bis. Quatre balles de fronde en plomb; Musée de Lattes; Feugère 1992, 140; L. 35, 38, 48 $\mathrm{mm}$. Deux d'entre elles proviennent de contextes datés, l'un du Ve s. av. n. ère, l'autre (u.s. 5001) du Ier s. av. ou ap. J.-C.

57. Bouton à anneau, Ier s.; u.s. 130055; Musée de Lattes; L. act. $15 \mathrm{~mm}$. V. ci-dessus l'objet de Corneilhan, $\mathrm{n}^{\circ} 41$.

58. Petit pendant, fouilles 1997, us 13013; L. act. $15 \mathrm{~mm}$. Cette forme de petit pendant percé est attestée localement à Aspiran et à Mèze; c'est sans doute un ornement de harnais ( $c f$. à Vindonissa: Unz, Deschler-Erb 1997, pl. 63, 1797-1798). 


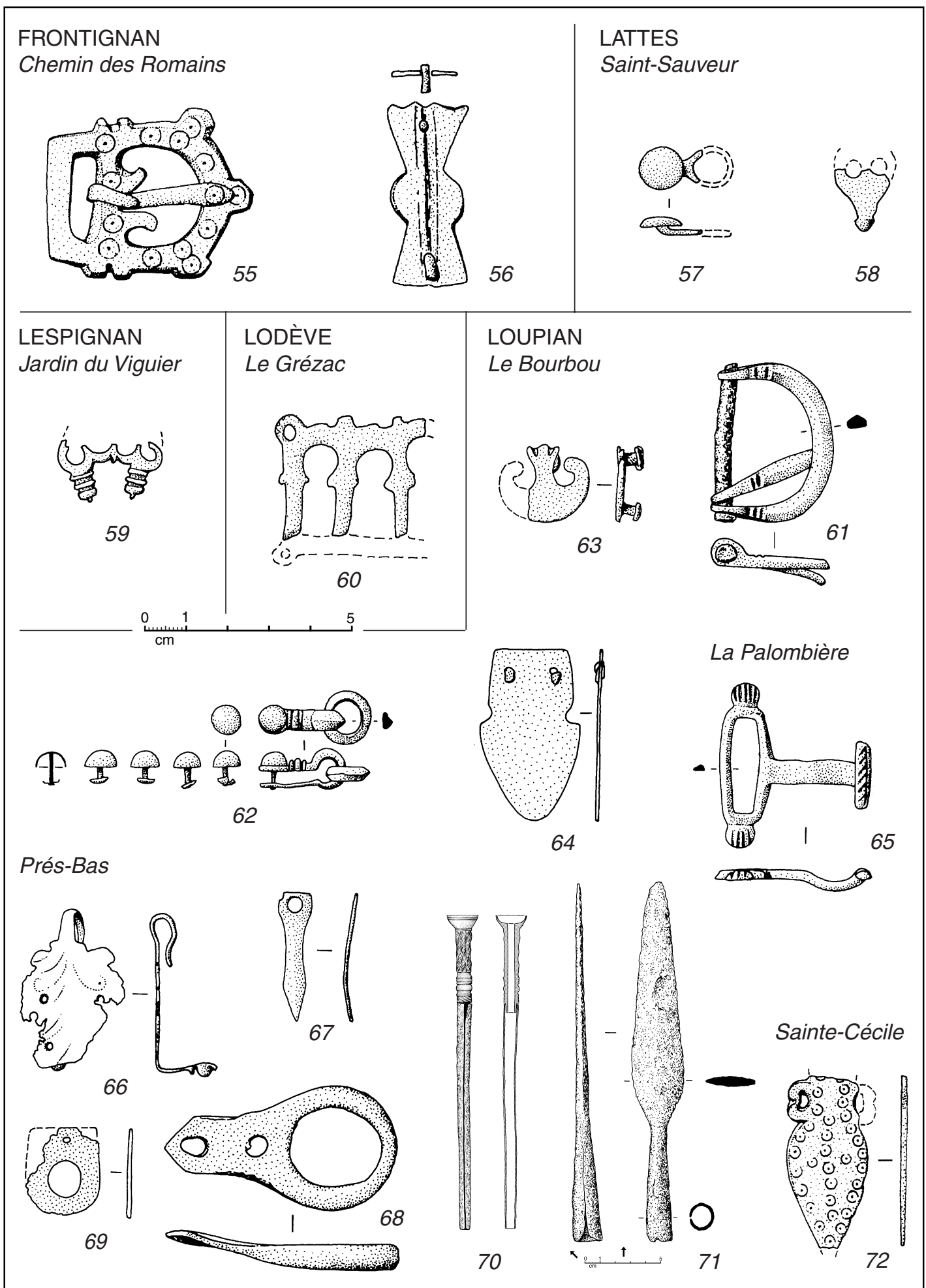

Fig. 12. Militaria du département de l'Hérault (v. le catalogue). Ech. 2/3. 
Lespignan (Fig. 12)

- Jardin du Viguier

59. Pendant de harnais, Ht-Emp.; prosp. et don G. Cros; MPM, inv. 2001.57.2; larg. 25 mm. Base à deux lests moulurés, appartenant à un pendant de harnais articulé: v. ci-dessous les découvertes de Saint-Saturnin $\left(n^{\circ} 168\right)$ et de Servian $\left(n^{\circ} 173\right)$.

Lodève (Fig. 12)

- Le Grézac

60. Fragment de plaque-boucle, IVe-Ve s.; Louis, Hébrard 1949, 97. Ce fragment de plaque à décor d'arcs outrepassés appartient à une plaque-boucle de type «Simancas», groupe III d'Aurrecoechea (2001: 94, de La Nuez de Abajo; fig. 66, $\mathrm{n}^{\circ} 2$ - Carpio de Tajo- à rivets coulés et fig. $66, \mathrm{n}^{\circ} 4$ - La Morterona- à rivets rapportés comme ici). Ce type peu répandu est daté en Espagne de la fin du IVe et du Ve s. de n. ère (ibid., 158).

Loupian (Fig. 12)

- Le Bourbou (atelier d'amphores et port commercial)

61. Boucle de harnais, contexte 50-80 ap. J.-C.; Feugère, Pellecuer 1998, fig. 2, 1; L. 38,5 x $29 \mathrm{~mm}$. Boucle en bronze, avec axe en fer, ayant conservé son ardillon de bronze. Type classique désormais rattaché au harnachement plutôt qu'au cingulum.

62. Agrafe et boutons de harnais, contexte 50-80 ap. J.-C.; Feugère, Pellecuer 1998, fig. 2, 2; L. de l'agrafe $20,7 \mathrm{~mm}$. Petite agrafe de lanière, ayant conservé son anneau de jonction en bronze et associée à cinq petits boutons hémisphériques, dont un lui sert de rivet. La position de ces 6 objets in situ, alignés côte à côte, sans espacement, indique que c'est la lanière elle-même qui a été enfouie dans le sol. Quelques lots de boutons similaires montrent que les boutons même isolés peuvent être avec certitude associés au harnachement: dans la tombe du Long-Pont à Thorembais-Saint-Trond, 33 exemplaires associés à d'autres éléments de ce harnais du IIIe s. (Mariën 1994, fig. 7, 24); à Celles-les-Waremme, en place sur une lanière de cuir, et à Han-sur-Lesse (cités par Mariën 1984, 22); à Vayres (Gironde), 23 boutons sont associés à une applique en forme de pelte (comme celle du Bourbou, ci-dessous), dans le dépotoir d'un four de potier comblé vers 250-320 ap. J.-C. (rens. Ch. Sireix). La morphologie de l'attache ( $c f$. par ex. à Velsen: Bosman 1995, fig. 6, 38), permet elle aussi de confirmer l'attribution au harnais de cavalerie (v. aussi un bouton isolé d'Ambrussum, daté du IIe s., ci-dessous $\mathrm{n}^{\circ} 213$ ).

63. Applique de harnais, contexte vers 400-425 ap. J.-C.; Feugère, Pellecuer 1998, fig. 2, 3; ht. $18,5 \mathrm{~mm}$. Applique en forme de pelte surmontée d'un fleuron: type classique, dont nous avons déjà signalé la réutilisation (?) sur les ceintures des IVe-Ve s. (Argeliers: Cathala 1903; Héron de Villefosse 1903).

64. Ferret de ceinture, contexte vers 400-425 ap. J.-C.; Feugère, Pellecuer 1998, fig. 2, 4; L. $41 \mathrm{~mm}$. Dos d'un ferret en deux parties de tôle accolées; la face antérieure devait être la seule à porter un décor; l'un des rivets de bronze assurant la fixation sur l'extrémité de la ceinture est encore en place. La construction du ferret en deux plaques distinctes, connue par quelques objets du Nord de la Loire (par. ex. Böhme 1974, pl. 147, 8), semble relativement fréquente sur les ferrets méditerranéens.

- La Palombière

65. Agrafe hispanique («pasador»); prosp. et coll. D. Rouquette; Feugère, Pellecuer 1998, fig. 2, 5; boucle de type «pasador en T»; L. 36,5mm.Cet objet recueilli hors contexte, en 1985 , à env. $300 \mathrm{~m}$ à l'Ouest de la villa des Prés-Bas, peut être mis en relation avec l'intense occupation de ce bassin-versant durant l'Antiquité. On notera la très grande ra- 
reté de ces boucles de ceinture, de facture ibérique (Palol 1955-56; Villaverde 1993), hors de leur zone de fabrication, le centre et le Sud-Est espagnols; on en a néanmoins signalé au Maroc (Boube 1994, $\mathrm{n}^{\circ}$ 89-96). La chronologie est indécise, allant selon les trouvailles du Ier s. av. n. ère à l'Antiquité tardive.

- Prés-Bas (grande villa gallo-romaine)

66. Pendant foliacé; fouilles Chr. Pellecuer, contexte c. 100-175 ap. J.-C.; Feugère, Pellecuer 1998, fig. 3, 12; L. act. $39 \mathrm{~mm}$. Contour découpé, décor poinçonné et ajouré; le lest en forme d'oignon est tordu vers l'arrière.

67. Pendant de tablier militaire (?); fouilles Chr. Pellecuer, contexte daté de 325-375 ap. J.C.; Feugère, Pellecuer 1998, fig. 3, 9; L. 32 mm. Malgré le contexte tardif, la typologie de tels pendants, bien datés de la deuxième moitié du IIe et du IIIe siècle, permet de voir dans cette trouvaille un objet résiduel (nombreuses découvertes sur le Mur d'Hadrien: AllasonJones 1988, fig. 4, 50 b2 et fig. 6, 52a8; Aldborough: Bishop 1996, nº 448; à Köngen: Luik 1996, pl. 47, 11-13; sur le limes: Oldenstein 1976, $\mathrm{n}^{\circ}$ 291-295; en Pannonie: Dawson 1990, fig. 2, 22-24; Petculescu 1995, pl. 1, 3-4; pl. 2, 2; Iža: Rajtàr 1994, fig. 8, 4). M.C. Bishop a néanmoins contesté leur attribution au «tablier» de cingulum, suggérant de classer plutôt ces objets parmi les pendants de harnais (Bishop 1992, 98-99).

68. Suspension de harnais; fouilles Chr. Pellecuer, contexte du IIIe s.; Feugère, Pellecuer 1998, fig. 3, 10; L. $57 \mathrm{~mm}$. Malgré sa facture robuste, inhabituelle, et la double perforation pour fixer la lanière de cuir, l'appartenance de cette suspension au harnachement ne fait aucun doute.

69. Suspension de phalère; fouilles Chr. Pellecuer, h.s.; Feugère, Pellecuer 1998, fig. 3, 11; L. $21 \mathrm{~mm}$. Contrairement à la précédente, cette suspension en tôle montre la forme classique des attaches associées aux diverses formes de pendants de harnais (v. également ici à Fontès, $n^{\circ} 48$ ).

70. Embouchure d'instrument à vent, en bronze; fouilles Chr. Pellecuer, contexte c. 100-175 ap. J.-C.; Feugère, Pellecuer 1998, fig. 3, 8; L. tot. 203 mm. La partie conservée se compose d'une embouchure coulée, longue de $63 \mathrm{~mm}$, au corps soigneusement orné de bourgeons et de moulures, venant s'emboîter dans un tube en tôle, ouvert, long de $147 \mathrm{~mm}$ et d'un diamètre interne de $6 \mathrm{~mm}$. Il semble s'agir d'une pièce interne assurant la jonction entre la pièce à bouche et le corps proprement dit, non conservé. Plusieurs embouchures d'instruments à vent (du type lituus ou cornu: v. Feugère 1993, 69-72) ont été trouvées en contexte militaire, mais ces objets connaissaient aussi des usages civils, publics (jeux) ou privés. La découverte de cet instrument dans une riche villa ne permet donc pas de préciser s'il s'agit ici d'un instrument militaire ou civil.

71. Pointe de lance en fer; fouilles Chr. Pellecuer, contexte c. 300-350 ap. J.-C; Pellecuer 1998, fig. 8, 2; Feugère, Pellecuer 1998, fig. 3, 7; L. $231 \mathrm{~mm}$. Pointe de lance en fer, à bords rectilignes: dans le contexte de la villa de Loupian, cette arme doit plutôt être considérée comme une arme de chasse que comme un objet militaire.

- Ste-Cécile (église et nécropole tardo-romaine et médiévale)

72. Ferret de ceinture, IVe s.; surface; dépôt de Loupian; Feugère 1993a, 253, 17; L. act. 40 $\mathrm{mm}$. Pendant en forme d'amphore, incomplet aux deux extrémités, dont le corps est simplement couvert de cercles ocellés, un traitement très apprécié sur les éléments de ceinture de Gaule méridionale.

Lunel-Viel (Fig. 13)

- nécropole

73. Ferret de ceinture, IVe s.; étude en cours (forme A de Sommer). 


\section{- habitat}

74. Pendant de harnais; fouilles et rens. Cl. Raynaud, Ier s.; inv. LV 300 / 2097; L. 74 mm. Pendant en cuivre rouge ayant conservé son décor gravé et niellé. Le type est original, mais on connaît un parallèle très proche à Alésia (Rabeisen 1990, fig. 12, 8; 1993, fig. 5, $\left.\mathrm{n}^{\mathrm{o}} 10\right)$.

Magalas (Fig. 14)

- Montfo (important oppidum de l'Age du Fer)

75. Pointe de flèche à trois ailerons (type 3 de Zanier); MPM, inv. 2000.27.1; L. $52 \mathrm{~mm}$. Ce type de pointe à soie et trois ailerons apparaît dans des contextes tardo-républicains, par exemple à Numance (Schulten 1927, pl. 34, 31-34) (Zanier 1988) et se rencontre ensuite jusqu'au IIIe s. de n. ère. Dans le contexte de cet oppidum, une datation précoce apparaît comme la plus vraisemblable. Dans l'Antiquité, les pointes de ce type sont des armes à usage exclusivement militaire.

76. Balle de fronde en plomb; prosp. et anc. coll. M. Elmi, Caraman; L. $41 \mathrm{~mm}, 76,3$ g. Type classique des balles de fronde du Ier s. av. ou ap. J.-C.: v. localement les exemplaires d'Aumes $\left(\mathrm{n}^{\mathrm{o}} 15\right)$ et de Pézenas $\left(\mathrm{n}^{\mathrm{o}} 123\right)$, ainsi que la découverte plus douteuse de Servian $\left(n^{\circ} 189\right)$.

Margon (Fig. 13)

- La Perrière (villa gallo-romaine)

77. Agrafe de harnais, Ht-Emp;; prosp. et coll. J.-L. Espérou; Depeyrot et al. 1986, fig. 23, 8; L. $40 \mathrm{~mm}$.

78. Agrafe de harnais, Ht-Emp.; prosp. et don J.-C. Rouanet; MPM, inv. 2000.37.1; L. 35,5 mm. $C f$. Vindonissa (Unz, Deschler-Erb 1997, n 1699-1700).

79. Applique en lunule, Ht-Emp.; prosp. et coll. P. Gauthier, Sommières; Depeyrot et al. 1986, fig. 23, 9; L. $30 \mathrm{~mm}$.

80. Pendant de harnais; prosp. et coll. J.-Cl. Rouanet; L. 48,5 mm. Pendant coulé, en forme de feuille terminée par un triple fleuron: modèle très classique qui connaît une large diffusion dans les provinces aux IIe-IIIe s. de n. ère (en Gaule à Tourtres, Lot-et-Gar., coll. J.-P. Noldin; Château-Porcien, Ardennes, rens. B. Lambot; Augst: Deschler-Erb, Schwarz 1993, fig. 5; en Germanie à Mayence: Bishop 1992, fig. 17, 9; en Bretagne à Cirencester: Webster 1982, no 0117; St-Albans: Frere 1984, 36 et fig. 12, 88; en Espagne à Albacete: Abascal, Sanz 1993, no 220; en Hongrie à Tihany: Deschler-Erb, Schwarz 1993, fig. 6). Certains d'entre eux (Mainz, Augst, Tihany) ont conservé la suspension à bouton qui était rivetée sur l'extrémité de la lanière.

- Les Piles (villa gallo-romaine)

81. Applique de harnais à bord ajouré; props. et don J.-Cl. Rouanet; MPM, inv. 2000.6.6; Abauzit 2000, fig. 2; L. act. 41,5 mm. V. ici-même à propos des appliques de Bessan (n ${ }^{\circ}$ $27)$ et d'Octon $\left(n^{\circ} 120\right)$.

\section{Marseillan (Fig. 13)}

- Les Belles (villa gallo-romaine)

82. Pendant de harnais, Ier s.; prosp. et don P. Brandelet; MPM, inv. 993.50.1; L. act. 38 $\mathrm{mm}$. Pendant foliacé dont le crochet évoque une tête d'oiseau aquatique.

83. Applique de harnais, Ier s.; prosp. et don P. Brandelet; MPM, inv. 994.75.1; L. 46,5 mm (traces d'argenture ou d'étamage).Il s'agit ici d'une garniture de lanière à la découpe caractéristique (type 6e de Bishop 1988): on relève en effet plusieurs variantes de ce motif symétrique parmi la production des ateliers d'Alésia (Rabeisen 1990, fig. 11). 


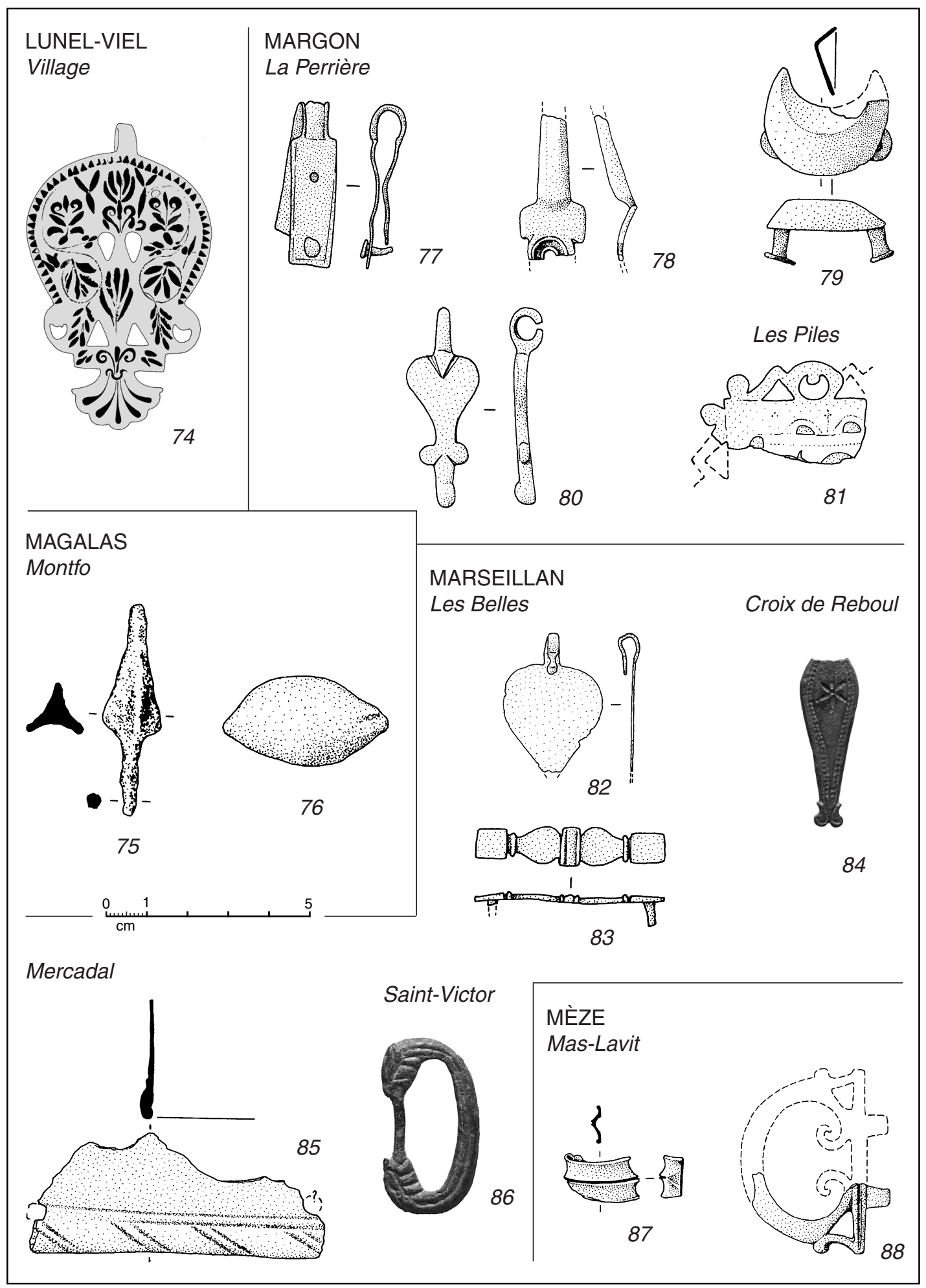

Fig. 13. Militaria du département de l'Hérault (v. le catalogue). Ech. 2/3. 
- Croix de Reboul (villa gallo-romaine)

84. Ferret de ceinture, IVe-Ve s.; prosp. et don P. Brandelet; MPM, inv. 993.52.2; L. act. 40 $\mathrm{mm}$. Partie inférieure d'un ferret massif, coulé, décoré en creux (estampage?) sur un fond plat étamé, une technique rarement attestée à cette époque: bordure perlée encadrant un fleuron central; à base, deux têtes d'oiseaux adossées.

- Mercadal (villa gallo-romaine sur habitat indigène)

85. Fragment de casque étrusco-italique en bronze, -Ier s.; prosp. et don J.-C. Rouanet; MPM, inv. 2000.70.6; L. act. $72 \mathrm{~mm}$. Bordure épaissie, ornée de doubles traits obliques, d'un casque étrusco-italique appartenant, soit au type ancien à bouton sommital (IIe s. av. n. ère), soit au type Mannheim, à calotte lisse (première moitié du Ier s. av. n. ère) (Schaaff 1988; Feugère 1994; Quesada 1997). Le fragment concerne le temporal arrière, avec la perforation latérale et l'amorce des moulures qui annoncent le couvre-nuque.

- Saint-Victor (nécropole gallo-romaine et du haut Moyen Age)

86. Boucle de ceinture; Lugand, Bermond (dir.) 2001, fig. 354; prosp. et don P. Gouyon; MPM, inv. 2000.41.4; L. $44 \mathrm{~mm}$. Cette boucle à extrémités zoomorphes stylisées possède toutes les caractéristiques des parures qui se rencontrent dans les premières tombes de l'Antiquité tardive à mobilier «germanique» de Gaule septentrionale (Böhme 1974); il est difficile de préciser si cette boucle équipait la plaque évidée d'une garniture en 5 parties, comme celle qui a été retrouvée à Alignan-du-Vent, ou un autre type contemporain.

Mèze (Fig. 13 et 14)

- Mas-Lavit (grande villa gallo-romaine)

87. Fragment de barrette de fourreau de glaive; Feugère, Pellecuer 1998, fig. 4, 14

88. Boucle de cingulum, Ier s.; prosp. et don P. P. Brandelet; MPM, inv. 993.47.5; Feugère 1994a, fig. 24, 2; Feugère, Pellecuer 1998, fig. 4, 13; L. 40 mm.

89. Pendant foliacé, Ht-Emp;; prosp. et don P. P. Brandelet; MPM, inv. 993.47.4; Feugère 1994a, fig. 24, 6; Feugère, Pellecuer 1998, fig. 4, 15; L. act. 45 mm.

90. Petit pendant foliacé, Ht-Emp; prosp. et don P. Brandelet; MPM, inv. 993.47.3; Feugère 1994a, fig. 24, 4; Feugère, Pellecuer 1998, fig. 4,16; L. 28,5 mm. Parallèles départementaux: Aspiran ( $\left.\mathrm{n}^{\circ} 10\right)$, Lattes $\left(\mathrm{n}^{\circ} 58\right)$, Sauvian $\left(\mathrm{n}^{\circ} 170\right)$ (type également attesté dans d'autres provinces, mais avec des variantes, par ex. à Cirencester: Webster 1982, $\mathrm{n}^{\circ} 116$ ).

91. Pendant de harnais, Ht-Emp.; prosp. ArchéOfactory, 1987; Feugère, Pellecuer 1998, fig. 4, 17; L. act. $28 \mathrm{~mm}$. Fragment semblant brisé au niveau d'une perforation transversale dans une moulure.

Minerve (Fig. 14)

- Le Pech (nécropole tardo-romaine et du haut Moyen Age)

92. Boucle de type Teba, IV-Ve s.; fouilles J. Lauriol, surf., début des années 50; Musée de Minerve; citée sans ill. dans Lauriol 1964-65, 187; L. $40 \mathrm{~mm}$. Boucle à volutes internes, fenêtre rectangulaire terminée par deux ergots arrondis. Entre les volutes, traces de l'ardillon en fer. Sur ce type, v. ci-dessus à propos de l'exemplaire de Frontignan, no 55.

Montady (Fig. 14)

- Agoutis

93. Applique émaillée; prosp. et coll. P. Abauzit; L. $42 \mathrm{~mm}$. Le décor central, corrodé, est transformé en masse jaune clair pulvérulente, mais les deux appendices latéraux en forme de pelte ont conservé leur émail vert. Les deux fixations du revers ressemblent plus à 


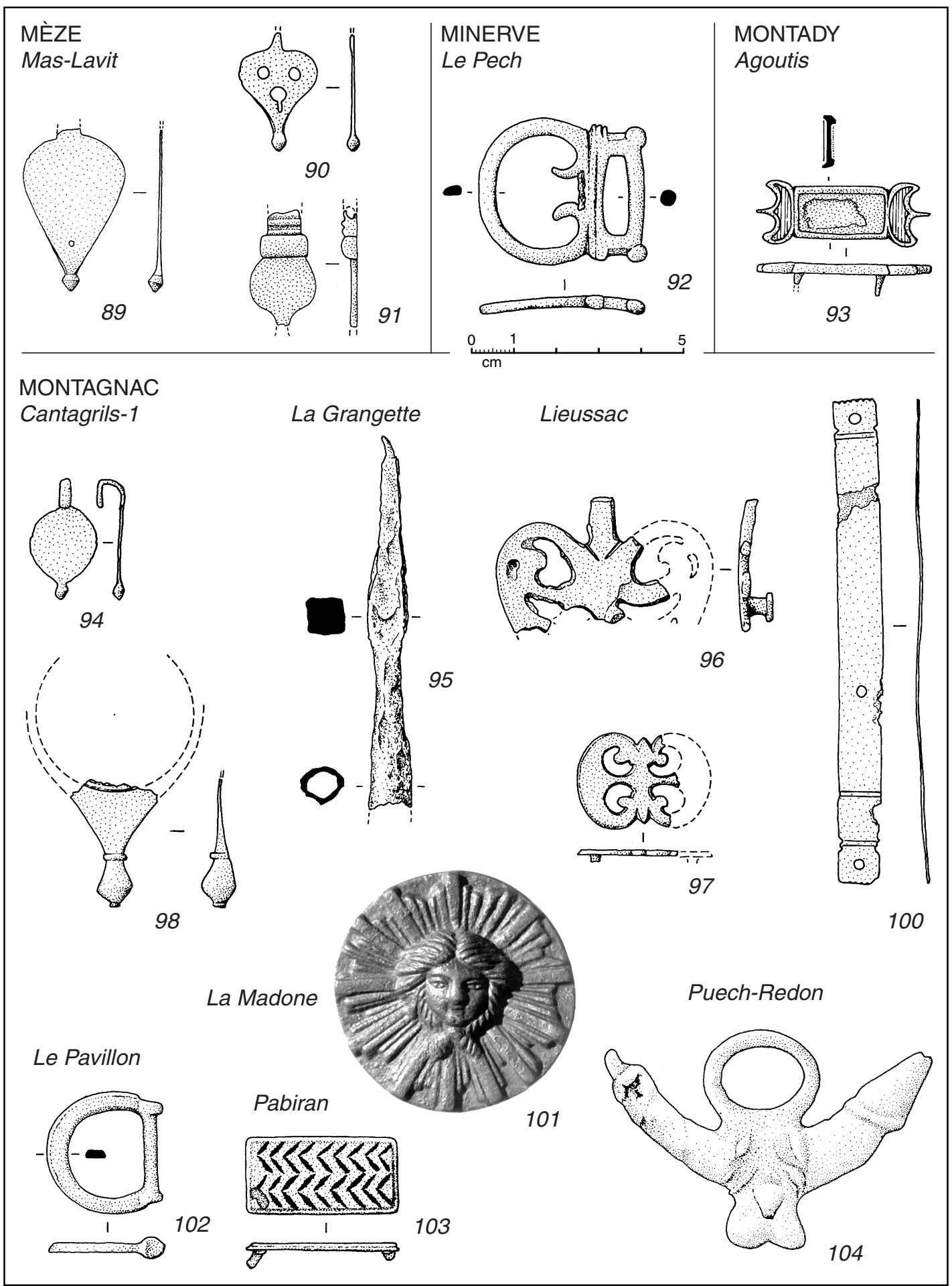

Fig. 14. Militaria du département de l'Hérault (v. le catalogue). Ech. 2/3. 
des pointes effilées qu'à des «rivets» pour cuir qui auraient perdu leur tête. La forme de cette applique et la présence d'appendices symétriques en forme de lunule renvoie aux productions émaillées de la fin du IIe et du IIIe s.

Montagnac (Fig. 14)

- Cantagrils-1 (habitat gallo-romain)

94. Petit pendant de harnais; prosp. et don P. Gouyon; MPM, inv. 2001.6.11; L. $31 \mathrm{~mm}$. Le site se trouve au contact de la voie domitienne, où l'objet a pu être perdu par un voyageur.

- La Grangette (habitat gallo-romain)

95. Trait de catapulte ou d'arbalète? Ht-Emp.; prosp. et don D. Dessalles; MPM; L. $88 \mathrm{~mm}$ (douille probablement dégradée); poids 20,37 g. La présence d'un trait de catapulte sur ce site rural étant des plus improbable, on peut considérer cet objet, s'il est bien antique comme semble le suggérer l'environnement, comme une armature de trait d'arbalète, une arme utilisée dans l'Antiquité pour la seule chasse (Baatz 1991); mais malgré les possibilités théoriques de distinction morphologique entre les deux séries de traits, cette démarche devrait s'appuyer sur des données de longueur et surtout de poids (une arbalète envoie des traits plus légers que la plus petite des catapultes) qui font actuellement défaut dans les publications.

- Lieussac (villa gallo-romaine)

96. Applique, Ht-Emp.; prosp. et don M. Feugère; MPM, inv. 993.19.24; L. act. 41,5 mm, restit. $51 \mathrm{~mm}$. Ce curieux décor d'applique, qui comporte encore au dos un rivet de fixation pour cuir, n'évoque aucun parallèle connu. Le décor d'ajours présente un aspect non ébarbé (objet en cours de fabrication?).

97. Applique de harnais, Ht-Emp.; MPM, inv. 994.7.105; L. act. 24, restit. 32 mm. Cette applique était constituée de deux peltes ajourées, reliées par leurs sommets. La fréquence de ce motif dans le domaine du harnais rend cette attribution la plus plausible (v. par ex. une série d'appliques de Wange: Lodewijckx 1993, fig. 8, 4.17).

98. Extrémité de pendant de harnais, Ht-Emp.; prosp. et don M. Feugère; MPM, inv. 993.19.25; L. act. $30 \mathrm{~mm}$. Base d'un pendant dont la plaque s'ornait de cercles incisés.

99. Talon de lance en fer; fouilles et don S. Mauné, surface; MPM, inv. 994.7.67. Cette découverte, effectuée sur un établissement rural, apporte-t-elle de l'eau au moulin de ceux qui considèrent que les talons coniques ont pu équiper certains outils, ou doit-elle être rapprochée des pointes de lances découvertes sur d'autres sites analogues du département (ici Loupian, $\mathrm{n}^{\mathrm{o}} 71$ et Pézenas, $\mathrm{n}^{\mathrm{o}} 125$; v. aussi le talon d'Ambrussum, $\mathrm{n}^{\mathrm{o}}$ 203)?

100. Bande-applique de ceinture, IV-Ve s.; fouilles et don S. Mauné, us 1001; MPM, inv. 994.51.1; L. 116, larg. 10,5 mm. Simple bande de tôle dont les extrémités percées sont échancrées et dentelées. Bien que la recherche de parallèles régionaux demeure infructueuse, il pourrait s'agir d'une variante locale des larges renforts de ceinture bien attestés dans les tombes «germaniques» de Gaule septentrionale (Böhme 1974, par ex. pl. 97, 12-14).

- La Madone (tombe gallo-romaine?)

101. Phalère en bronze; coll. M. Benezeth, Montagnac; Mauné 1998, fig. 134; diam.? Cet objet de qualité, en bronze étamé ou argenté, comporte au revers les deux rivets caractéristiques d'une fixation sur harnais de cuir. Il peut donc s'agir d'une décoration (donum militare) ou d'un décor de harnais. La tête juvénile du motif central, dont les nattes semblent nouées sous le cou, est placée au centre d'un motif rayonnant qui semble original dans l'iconographie antique. 
- Le Pavillon (habitat gallo-romain)

102. Boucle en bronze, Haut-Empire?; MPM, inv. 994.94.1; L. 27, larg. 29,5 mm. Boucle massive, à traverse en bronze, le tout semblant coulé d'un seul tenant; bords chanfreinés; l'attribution au harnais semble la plus vraisemblable.

- Pabiran (grande villa gallo-romaine, puis habitat et nécropole du haut Moyen Age)

103. Applique de cingulum, Ier s.; prosp. et don G. Jacquet / M. Feugère (San Peyre); MPM, inv. 989.60.1; dim. 37 x 18,5 mm. Applique à 4 rivets, ornée d'une double rangée de chevrons niellés; chaque chevron est constitué de deux feuilles au pourtour dentelé, un motif particulièrement fréquent dans l'ornementation de l'équipement militaire du Ier s. (Deschler-Erb 2000).

Plaque rectangulaire allongée, pourvue au revers de 4 pointes pour fixation; bordure moulée, décor de deux lignes horizontales de chevrons niellés.

- Puech-Redon (habitat gallo-romain - mansio?)

104. Pendant phallique, Ht-Emp.; Feugère 1981, 144, fig. 8a (sous une provenance erronée); Depeyrot et al. 1986, fig. 29, B1; L. $77 \mathrm{~mm}$. Ce type de pendant symétrique connaît plusieurs variantes, dont une première classification a été tentée par M. Bishop (1988, type 10). Leur attribution au domaine du harnachement est confirmée par quelques exemplaires qui ont conservé la suspension caractéristique, comme par exemple à Eauze (Arramond et al. 1986, 22, fig. 11, 7)

\section{Montpellier (Fig. 15)}

- St-Michel (nécropole tardo-romaine)

105. Boucle de ceinture, IV-Ve s.; Majurel et al. 1970, fig. 19, 1; Feugère 1993b, 145, fig. 14, 6; L. $38 \mathrm{~mm}$. Cette forme de boucle à bords concaves, avec ou sans globules angulaires, est typiquement romaine: elle apparaît dans des contextes militaires tardorépublicains (Ulbert 1984, pl. 10, 62; Poux 1999, 63-80) et, se décline dans les provinces du Haut-Empire, notamment en Pannonie, sous des formes diverses (Böhme 1978, carte fig. 75).

\section{Montpeyroux (Fig. 15)}

- Les Thérons (habitat gallo-romain)

106. Fragment de boucle à dauphins, IV-Ve s.; prosp. et don G. Cabrol; MPM, inv. 993.53.1; L. act. $29 \mathrm{~mm}$. Fragment d'une grande boucle à dauphins affrontés, décorée de cercles ocellés.

\section{Mourèze (Fig. 15)}

- lieu-dit?

107. Plaque-boucle rigide, IV-Ve s.; prosp. et don Ch. Berthomieu; MPM, inv. 2000.23.1; L. $42 \mathrm{~mm}$. Boucle associée à une plaque dont l'extrémité reprend le motif des appliques de ceinture «en forme d'hélice». Ce type connaît plusieurs parallèles en Gaule septentrionale: Vermand, Vert-la-Gravelle, Champdolent... (Bullinger 1969, fig. 13, 1; $15,2 ; 16,2)$. On peut donc supposer que l'on est en présence d'une importation.

\section{Murviel-lès-Montpellier}

- Le Castellas (oppidum protohistorique et agglomération gallo-romaine)

108. Plaque de cingulum, Ier s.; coll. part. Simple décor de cercles concentriques effectués au tour. 
108 bis. Armature de cornu? Musée de Montpellier.

Nébian (Fig. 15)

- Pichaurès (habitat gallo-romain)

109. Applique en coquille, Ht-Emp.; prosp. et coll. G. Jacquet; Depeyrot et al. 1986, fig. $31 \mathrm{c}$; L. $23 \mathrm{~mm}$. L'appartenance de ce type d'applique au harnais est illustrée par de nombreuses trouvailles, dont la plus spectaculaire est une bride du tumulus de Celles, en Belgique: 40 appliques de ce modèle y apparaissent en place sur le cuir exceptionnellement conservé (Massart 2000, fig. 10).

\section{Neffiès (Fig. 15)}

- La Vérune (villa gallo-romaine sur habitat indigène)

110. Bouton à anneau à décor niellé, Ier s.; prosp. et don G. Jacquet; MPM, inv. 989.84.1; diam. 14, L. restituée $27 \mathrm{~mm}$. Tête bombée ornée de 6 traits niellés formant un fleuron. Tout en demeurant très rare sur ces objets (Vindonissa: Unz, Deschler-Erb 1997, pl. 71, $\mathrm{n}^{\mathrm{o}}$ 2060), le décor niellé fait partie des techniques utilisées sur les pièces d'équipement militaire du Ier s., notamment sur la ceinture et, dans une moindre mesure, le harnachement (Deschler-Erb 2000).

111. Agrafe de harnais, Ier s.; prosp. et don G. Bagan; MPM, inv. 2002.2.1; L. act. 38,5 $\mathrm{mm}$. Agrafe du style fortement mouluré, bien connu sur les sites militaires et localement attesté par les découvertes de Pomerols ( $\left.\mathrm{n}^{\mathrm{o}} 126\right)$ et de Vendres.

112. Bouton émaillé, IIIe s.; Depeyrot et al. 1986, fig. 37, 6; diam. 15 mm, L. act. 9,5 mm. Bord guilloché; décor de pâte de verre verte dans un émail oxydé.

113. Boucle à dauphins, abandonnée en cours de fabrication; MPM, inv. 988.65.1; Feugère 1993a, 253, 6; 1996b, 277, $\mathrm{n}^{\circ}$ 6; L. 44,5 mm. Ce document de grande importance atteste, d'une part, la fabrication d'objets de ce type sur cet établissement gallo-romain du Languedoc central, alors qu'une telle production ne ressort pas de l'état actuel de la recherche sur ces objets et leur répartition. Il permet en outre d'observer le mode de fabrication, dans un moule à cire perdue, alimentée par un unique canal latéral, sans évents, qui s'est fendu sous l'effet de la chaleur. De ce fait, la quantité d'alliage prévue par le bronzier s'est avérée insuffisante et l'objet, incomplètement moulé, a dû être rejeté.

- Site à préciser (peut-être le précédent)

114. Phalère en bronze; prosp. et coll. G. Jacquet; copie à la MPM; diam. $51 \mathrm{~mm}$. Elle représente une tête de Jupiter Ammon (cornes de bélier) sur un disque plat, avec une bordure légèrement moulurée. Le thème, attesté localement sur le bouton à anneau de Caux (cidessus, $\mathrm{n}^{\circ} 30$ ), est fréquemment utilisé en contexte militaire et notamment sur les phalères, par exemple à Lauersfort (Jahn 1860, pl. I, 4; Maxfield 1981). Autres thèmes sur des objets de même forme: Méduse, sur une phalère du lot d'objets de harnais de Wange, IIIe s. (Lodewijckx 1993, fig. 5, 4.1).

\section{Nissan-lez-Ensérune (Fig. 15)}

- Oppidum (important oppidum préromain occupé jusqu'au Ier s. de n. ère)

115. Double anneau à ergot; fouilles anciennes; Musée d'Ensérune, inv. OS-562; L. act. 35, restit. $45 \mathrm{~mm}$. Ces anneaux doubles, très fréquents en Italie mais rares ailleurs (Manching: Jacobi 1974, 192-195, $\mathrm{n}^{\circ} 818$ et 819 [ce dernier en fer], avec parallèles hors d'Italie; van Endert 1991, 74-75, n 384), ont longtemps été décrits comme des «doigtiers d'archer». Il semble que G. Jacobi (loc. cit.) ait été l'un des premiers à les attribuer à 


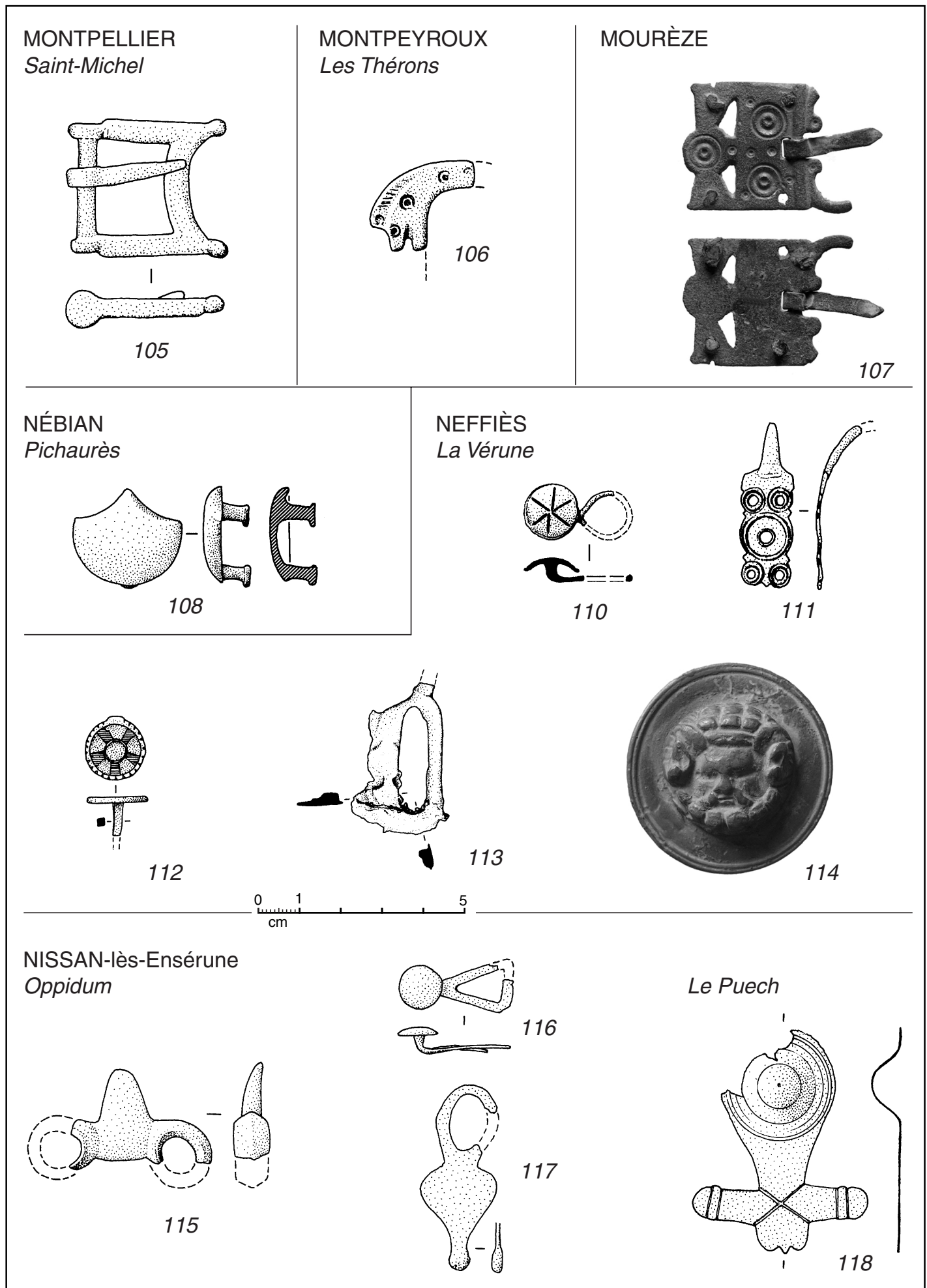

Fig. 15. Militaria du département de l'Hérault (v. le catalogue). Ech. 2/3. 
juste titre au harnais de tête d'un animal de trait. Selon R. Gebhard, qui a observé un exemplaire orné d'un bucrâne, c'est, du reste, à un harnais de bœuf plutôt que de cheval qu'il faudrait attribuer ces anneaux. Si leur datation tardo-républicaine, tout comme leur origine méditerranéenne, ne fait aucun doute, leur utilisation, militaire ou civile, demeure incertaine.

116. Bouton à anneau; Musée d'Ensérune, inv. OS-378; L. $28 \mathrm{~mm}$. Anneau plat de forme triangulaire: cette particularité, qui disparait ensuite au profit des anneaux circulaires, caractérise une forme ancienne de ces objets: époque augustéenne?

117. Pendant de harnais; Musée d'Ensérune, inv. 53-407; L. $45 \mathrm{~mm}$. Pendant foliacé, bouton épaissi mais informe, large anneau de suspension lui-même aplati. Les pendants à boucle fermée placée dans le plan de l'objet ne sont nulle part très répandus (v. cidessous, Le Pouget $\mathrm{n}^{\mathrm{o}}$ 130, Villetelle $\mathrm{n}^{\mathrm{o}}$ 196, et fig. G; également à Augst: DeschlerErb 1999, n 434).

- Le Pech (habitat gallo-romain)

118. Pendant de harnais, type à crochet; prosp. et coll. P. Abauzit. Pendant en tôle comportant une partie circulaire ornée d'une bosse en fort relief et de cercles circulaires, et un lest en forme de double phallus encadrant une représentation schématique des parties génitales masculines.

- La Paillette (habitat gallo-romain)

119. Boucle de harnais, Ier s.; prosp. et don G. Fédière; MPM, inv. 993.13.1;

Octon (Fig. 16)

- Terafort (habitat gallo-romain)

120. Applique de harnais, Ht-Emp.; prosp. et coll. E. Maistre; Schneider, Garcia 1998, fig. 193a; L. $112 \mathrm{~mm}$. Applique rectangulaire à bordure ajourée, portant deux fixations au revers. Elle figure Hercule au repos et se rattache aux appliques désormais attribuées au harnais romain, et relativement bien attestées en Gaule du Sud (Abauzit 2000) comme dans d'autres provinces (Božič 2001); v. dans l'Hérault les fragments de Bessan $\left(n^{\circ} 27\right)$ et de Margon $\left(n^{\circ} 81\right)$.

Paulhan (Fig. 16)

- Vareilles (villa gallo-romaine)

121. Extrémité de bouterolle?; prosp. et coll. P. Gauthier, Sommières; Depeyrot et al. 1986, fig. 45, a2; L. $23 \mathrm{~mm}$. Bouton mouluré et côtelé, coulé sur une tige de fer: extrémité d'une bouterolle de glaive? (Ulbert 1970, pl. 23, 343).

Pézenas (Fig. 16)

- L'Auribelle-Basse (villa gallo-romaine)

122. Bouton émaillé, IIe s.; prosp. et don D. Nepivoda; MPM, inv. 999.1.2; diam. 20 mm, L. act. 7,5 $\mathrm{mm}$. Trois zones émaillées, le cœur orangé avec dans la couronne externe les restes de pavés bleu foncé dans de l'émail vert (v. ci-dessus le bouton de Neffiès, $\mathrm{n}^{\circ}$ 112).

- Balsède-3 (petit habitat indigène, occupé jusqu'au Ier s. de n. ère)

123. Balle de fronde en plomb; prosp. et coll. G. Cabrol; Depeyrot et al. 1986, fig. 43, 2; copie à la MPM, inv. 990.56.1e' L. $45 \mathrm{~mm}$, poids $75 \mathrm{~g}$. Découverte à rapprocher de celles de Lattes (Feugère 1992), d'Aumes ( $\left.\mathrm{n}^{\mathrm{o}} 15\right)$ et de Magalas ( $\left.\mathrm{n}^{\mathrm{o}} 76\right)$. 


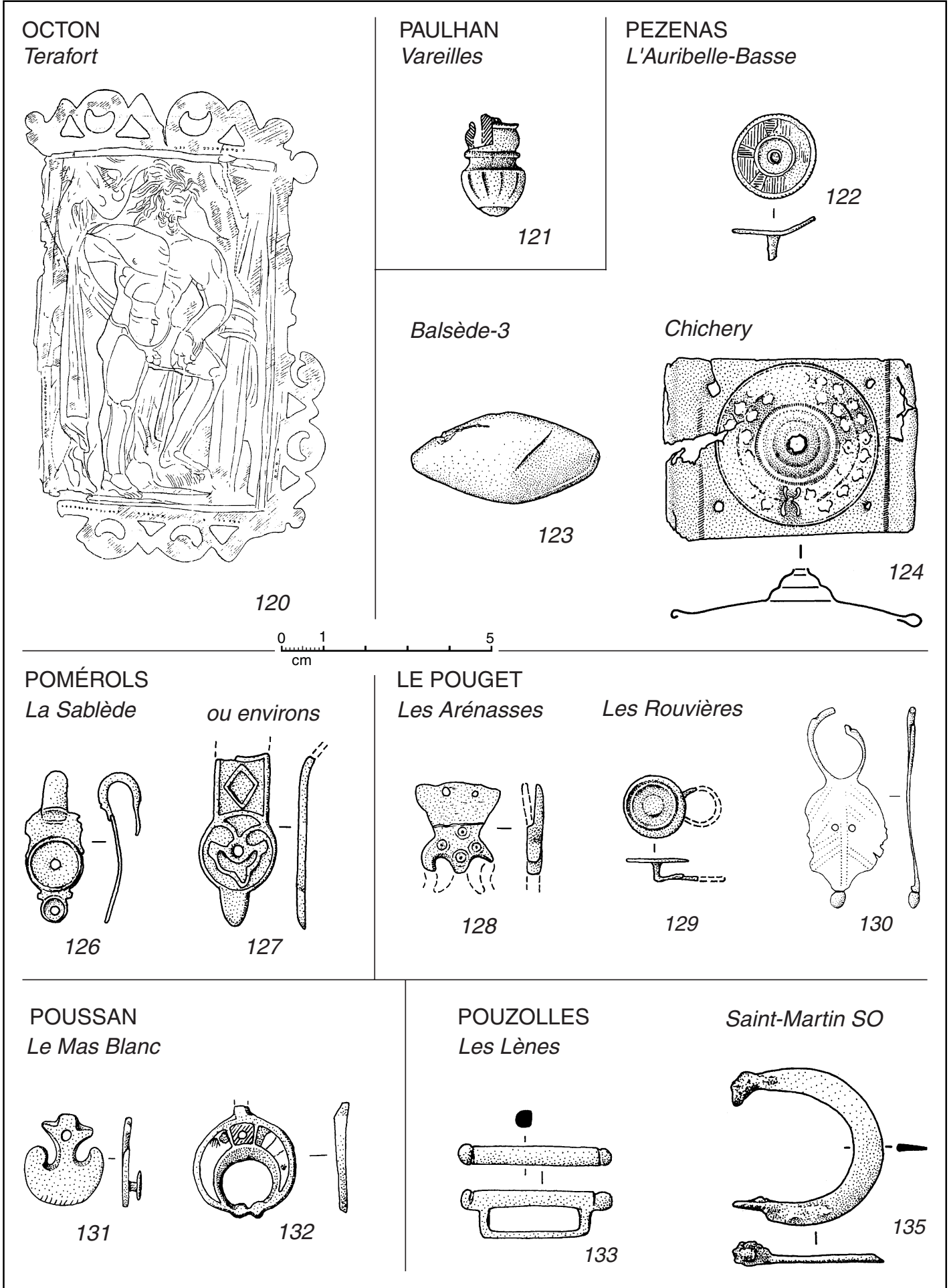

Fig. 16. Militaria du département de l'Hérault (v. le catalogue). Ech. 2/3. 
- Chichery (villa gallo-romaine)

124. Plaque de cingulum, Ier s.; MPM, inv. 989.65.3; Depeyrot et al. 1986, fig. 40, 10; Plaque en tôle, extrémités repliées sur elle-même; 4 trous de fixation pour rivets disparus; umbo central à 2 degrés, sommet percé. Décor incisé: couronne nouée, deux rangs de feuilles de lierre, fond chagriné.

- Roquelune (villa gallo-romaine)

125. Pointe de lance; MPM; prosp. et don S. Mauné; MPM. Comme à Loupian, cette armature de lance peut avoir été une arme de chasse, au moins dans son utilisation locale.

Pomérols (Fig. 16)

- La Sablède (habitat gallo-romain)

126. Agrafe de harnais, Ier s.; prosp. et don J.-C. Rouanet; MPM, inv. 2000.15.1; L. 36 mm.

- Pomérols, ou env.

127. Applique émaillée, IIIe s.; prosp. et don P. Gouyon; MPM, inv. 999. 10.3; L. act. 42 $\mathrm{mm}$. Bande terminée par un disque percé pour la fixation; les émaux champlevés dessinent des motifs géométriques sur la bande et une feuille de lierre (motif fréquemment utilisé sur le harnais) sur le disque.

Le Pouget (Fig. 16)

- Les Arénasses (habitat gallo-romain)

128. Ferret en forme d'amphore, IVe-Ve s.; prosp. et don G. Cabrol; MPM, in. 995.85.1; L. act. $21,5 \mathrm{~mm}$. Sommet riveté d'un pendant coulé, épais, dont la face antérieure était couverte d'un décor composé d'une juxtaposition de cercles oculés.

- Les Rouvières ( ou «Les Rouvièges»: habitat gallo-romain)

129. Bouton à anneau émaillé, IIe s.; prosp. et don G. Cabrol; MPM, inv. 994.76.1; L. act. $18 \mathrm{~mm}$.

130. Pendant de harnais; prosp. et don G. Cabrol; MPM, inv. 993.63.2; L. 43 mm. Pendant foliacé, à décor poinçonné et percé. Ce type de fixation, un anneau dégagé dans la masse de l'objet à la place du crochet recourbé habituel, ne semble guère fréquent endehors de la Gaule méridionale (par ex. ici n 117 ou 196; à Brignon ou St-Côme, Gard, coll. Rappaz); il n'est pas répertorié par M.C. Bishop dans son inventaire des types de pendants de harnais (1988).

\section{Poussan (Fig. 16)}

- Mas-Blanc (habitat gallo-romain)

131. Lunule émaillée, II-IIIe s.; prosp. et coll. A. Rougerie; Feugère, Pellecuer 1998, fig. 5, 18; L. 29,5 mm. Objet de fonction indéterminée ( $c f$. Albacete: Abascal, Sanz 1993, $\mathrm{n}^{\circ}$ 183), mais souvent attribué au harnachement quand il fait partie d'appliques complexes pourvue des rivets caractéristiques de la fixation sur cuir.

132. Applique en pelte, IIIe-Ve s.; prosp. et coll. A. Rougerie; Feugère, Pellecuer 1998, fig. 5,$19 ;$ L. $22 \mathrm{~mm}$. Un des rivets du revers, brisé, a fait l'objet d'une réparation.

Pouzolles (Fig. 16)

- Les Lènes (habitat gallo-romain)

133. Passant de harnais, Ier s.; prosp. et don G. Cabrol; MPM, inv. 2000.7.3; L. 37 mm. V. ci-dessus la découverte de Clermont-l'Hérault $\left(\mathrm{n}^{\circ} 40\right)$. 
- Reyne-Martre (villa gallo-romaine)

134. Pendant de harnais, IIe s.; coll. H. Leyris; pendant coulé à extrémité trifoliée, d'un type courant et attesté dans l'Hérault, par exemple, à Margon, La Perrière (v. ci-dessus nº 68).

- St-Martin-Sud-Ouest (grande villa gallo-romaine)

135. Boucle de harnais, Ier s.; prosp. et coll. J.-P. Mailhé; Depeyrot et al. 1986, fig. 48, 4; L. $37 \mathrm{~mm}$.

\section{Quarante (Fig. 17)}

- La Condamine (habitat gallo-romain)

136. Pendant de harnais, type à crochet; prosp. C. Lacombe; Musée de Quarante; ht. act. 36 $\mathrm{mm}$, larg. $44 \mathrm{~mm}$. La suspension est brisée: sur ce type soigné, elle pouvait avoir la forme d'une tête de canidé; l'objet montre les restes d'un décor finement ponctué.

- Massale (habitat gallo-romain)

137. Applique latérale de mors; prosp. C. Lacombe; Musée de Quarante; L. act. $44 \mathrm{~mm}$. Seule est conservée la base de cet objet ajouré, de facture robuste mais travaillé en ajours et comportant d'un côté un large passant rectangulaire: ces caractéristiques correspondent à une applique latérale de mors, que le style décoratif permettrait de placer au IIIe s., date à laquelle ces objets sont à la fois abondants et de formes variées (v. ici: Espondeilhan et Villetelle).

138. Pendant de harnais, type à crochet; prosp. C. Lacombe; Musée de Quarante; L. act. 42 $\mathrm{mm}$.

- Milliade (habitat gallo-romain)

139. Boucle de harnais, Ht-Emp.; Musée de Quarante.

- Parazols (villa gallo-romaine)

140. Boucle de harnais, Ier s.; Musée de Quarante.

141. Pendant de harnais, en forme de feuille; Musée de Quarante; ht. act. $35 \mathrm{~mm}$, larg. act. $32 \mathrm{~mm}$. La partie centrale se termine par une plaque transversale moulurée et une lunule (forme de lest très inhabituelle); le pendant, qui comportait aussi deux appendices latéraux terminés par de petites feuilles, a conservé son décor poinçonné, d'inspiration florale. Un des meilleurs parallèles disponibles est fourni par un objet de provenance malheureusement imprécise («Gaule méridionale») 7 , conservé à Montagnac (fig. 3, 4); v. également à Servian, $\mathrm{n}^{\circ} 176$.

142. Fragment d'applique émaillée; prosp. et don Ch. Lacombe; Musée de Quarante; L. act. $21 \mathrm{~mm}$. Applique rectangulaire à bords concaves, brisée en deux; le décor était constitué de trois bandes émaillées imbriquées les unes dans les autres. Les appliques émaillées à bords concaves montrent - mais surtout en Gaule du Nord - une grande variété (Fauduet 1992, 108).

143. Fragment d'applique de harnais en forme de pelte (fleuron sommital); prosp. et don Ch. Lacombe; Musée de Quarante; L. act. $14 \mathrm{~mm}$.

144. Bouton circulaire pour harnais; prosp. et don Ch. Lacombe; Musée de Quarante; diam. $25 \mathrm{~mm}$. Objet bien connu sur les harnais du IIIe s., comme Feurs (Feugère 1983) ou Wange (Lodewijckx 1993, fig. 6).

- Semèges (villa gallo-romaine)

145-146. Deux boutons de harnais émaillé; prosp. et don M. Serbera; Musée de Quarante; diam. 35,5 et $36 \mathrm{~mm}$. Ces deux boutons de module presque identique ont conservé une

7 Mais la mention «Zill», portée au crayon au revers de l'objet, renvoie sans doute à sa provenance exacte; MPM, inv. 997.45.7. 
partie de leurs émaux; sur le premier, le décor s'organise de manière classique en 3 bandes concentriques autour d'un point central (à l'extérieur, 16 carrés de verre bleu, pointés de rouge, fixés par des bandes d'émail, et décor identique dans la bande centrale; entre les deux, décor différent - seul subsiste un pavé de verre jaune; le point central est orange); sur le second, plus original, 6 cercles d'émail — pointés de rougenoyés dans une large bande; couronne étroite autour du point central, qui était jaune avec un point rouge. De larges boutons de ce type se retrouvent sur certains harnais du IIIe s., comme celui de Wange (Lodewijckx 1993, fig. 5, 4.3).

\section{Roujan (Fig. 17)}

- Le Colombier (habitat gallo-romain sur établissement indigène)

147. Extrémité de pendant de harnais, Ier s.; prosp. et don G. Cros; MPM, inv. 995.77.3; L. act. $26 \mathrm{~mm}$. Lest en forme d'oignon caractéristique des pendants à crochet de la première moitié du Ier $\mathrm{s}$. de $n$. ère.

- St-Nazaire (grande villa gallo-romaine, puis église et nécropole du haut Moyen Age)

148. Ferret de ceinture, IVe-Ve s.; MPM, inv. 989.15.2; L. act. 34 mm. Plaque trapézoïdale, ajoutée d'une pelte, pourvue au revers de trois rivets coulés, articulée (axe en fer) sur le sommet d'un pendant en forme d'amphore, orné de cercle(s) pointé(s). La forme générale du ferret est donnée par un objet presque superposable d'Aquileia (Bullinger 1972, pl. XV, 6). L'intérêt de cette découverte vient du mode de fixation du ferret sur la ceinture, par le biais d'une plaque articulée. Les ferrets de ce type, nettement moins répandus que les ferrets rivetés, semblent originaires d'une zone située au Nord de l'Adriatique. Il s'agirait donc ici d'une importation orientale.

- St-Jean (vicus gallo-romain)

148 bis. Ferret en bronze; prosp. et don G. Bagan; MPM, inv. 2002.9.13: L. act. 43,5 mm. Ferret foliacé, la suspension manque; décor uniface: cercles oculés et bordure hachurée.

Saint-André-de-Sangonis (Fig. 17)

- Sainte-Brigitte (établissement gallo-romain)

149. Applique de harnais; prosp. et don G. Cabrol; MPM, inv. 992.86.1; L. 40 mm. Restes d'étamage ou d'argenture. Applique symétrique

\section{Saint-Maurice-de-Navacelles (Fig. 17)}

- Camp des armes (nécropole tardo-romaine)

150. Boucle à extrémités zoomorphes, IVe s.; tombe $n^{0}$ 2, dr.; Arnal, Milhau 1964, fig. 3, 2 (dr.); Feugère 1993a, 253, 8; L. $50 \mathrm{~mm}$. Avec ses têtes aux contours très découpés, cette boucle relève d'un style différent des objets comparables retrouvés dans le département et, d'une manière générale, en Gaule méridionale. Compte tenu également du contexte, on peut sans doute la considérer comme une importation septentrionale.

151. Boucle à plaque ocellée; tombe ${ }^{\circ}$ 1; Arnal, Milhau 1964, fig. 3, 2 (gauche); L. 49 mm. Cette boucle, qui connaît d'innombrables parallèles en Gaule septentrionale (type A de Sommer), constitue pour le moment une découverte isolée dans le département; on notera du reste sa localisation excentrée, sur une voie de communication entre le littoral et le Massif Central.

152-156. Appliques de ceinture; tombe no 3; Arnal, Milhau 1964, fig. 3, 4; L. 37 x 12 mm. Retrouvées au niveau de la taille, mais sans autre élément de ceinture, ces appliques appartiennent à un modèle très simple, mais généralement mal daté (Vieil-Evreux: Fauduet 1992, $\left.\mathrm{n}^{\mathrm{o}} 713,720,721\right)$. 


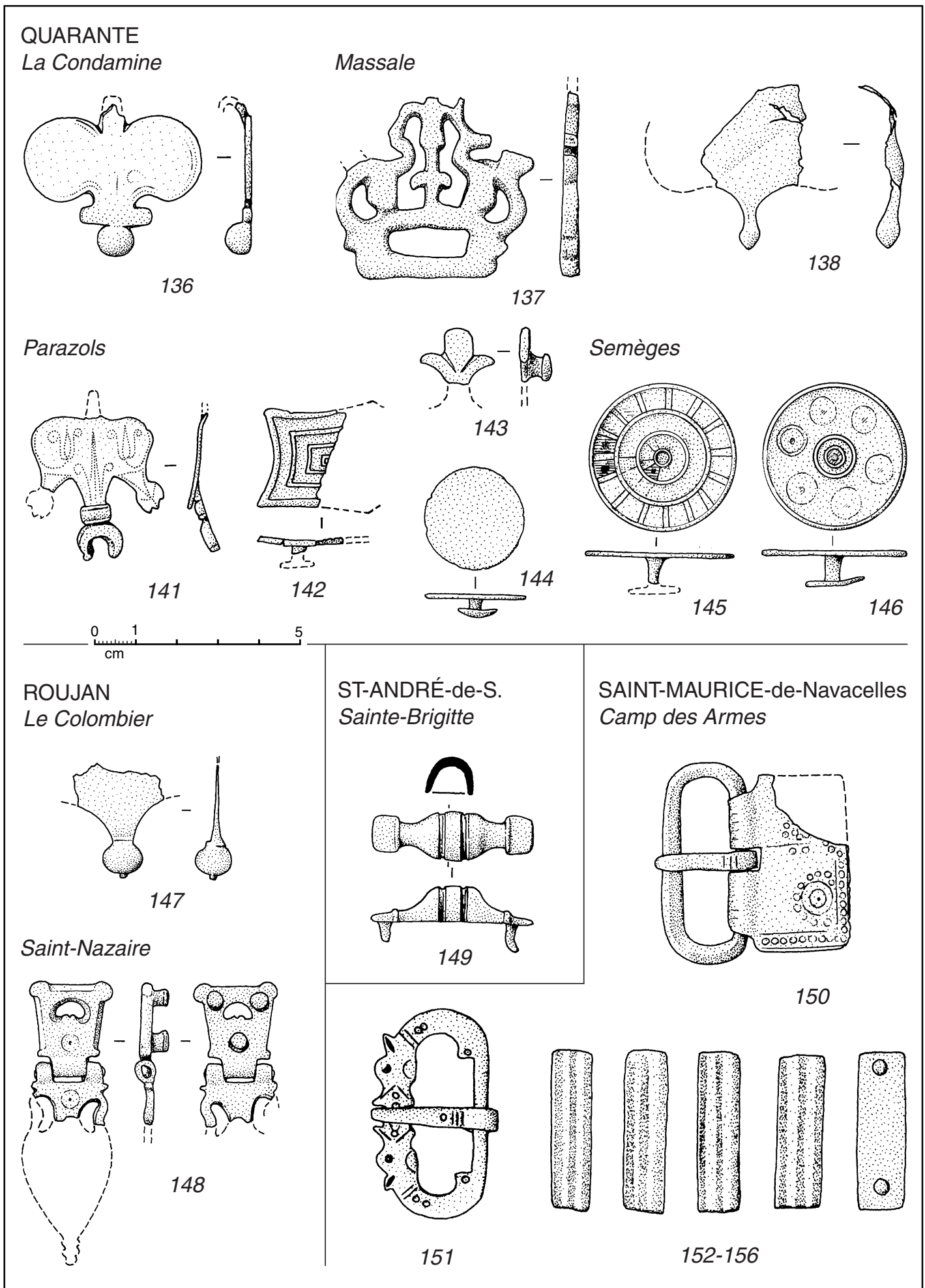

Fig. 17. Militaria du département de l'Hérault (v. le catalogue). Ech. 2/3. 


\section{Saint-Pargoire}

- Contour (atelier de potier et habitat gallo-romain)

157. Pendant harnais, Ier s.; prosp. et don M. Feugère; MPM. Crochet isolé, en forme de tête de canidé, d'un pendant de harnais du Ier s. de n. ère.

Saint-Pons-de-Mauchiens (Fig. 17)

- Caissergues

158. Plaque-boucle à dauphins, Ve s.; prosp. et don M. Feugère; MPM, inv. 993.34.1; Feugère 1994a, 18, fig. 27; L. 53 mm. Plaque-boucle rigide, à décor ocellé et motifs de dauphins sur la boucle; les rivets de fixation coulés, que l'on rencontre habituellement sur les objets de ce type en Gaule du Sud, sont remplacés ici par des perforations. Cet élément de ceinture original peut être considéré comme une production locale du Ve s. de n. ère.

Saint-Saturnin (Fig. 18)

- Aulas (très grand habitat — agglomération? — gallo-romain sur établissement indigène)

159. Applique de harnais, Ier s.; prosp. et don H. Leyris; MPM, inv. 994.66.18; L. 24 mm. On reconnaît un motif habituel des garnitures de harnais, décliné sous diverses formes dans des ateliers comme Alésia (Rabeisen 1990, fig. 11).

160. Pendant de harnais, type à crochet, prosp. et coll. Chr. Berthomieu; larg. 82,3, ht. 67,3 $\mathrm{mm}$. Grand pendant à crochet en forme de tête d'oiseau aquatique, avec lest biconique, ayant conservé sur la face externe, qui est étamée, un décor poinçonné et gravé d'inspiration végétale.

161. Fragment de caveçon en bronze, Ht-Emp.; prosp. et don G. Jacquet; MPM, inv. 989.36.5; Feugère 1993a, 180; L. act. 106 mm. Fragment comportant la moitié d'une bande nasale, élargie vers le centre et renforcée d'une épaisse côte médiane, ainsi que l'un des anneaux latéraux, accosté de deux ergots; comparer avec l'exemplaire sans doute plus ancien de Tourbes, $\mathrm{n}^{\circ} 178$.

162. Applique en pelte, III-Ve s.; MPM, inv. 994.4.12; L. $21 \mathrm{~mm}$. Applique mal ébarbée, semble inachevée (ouverture déficiente des creux de la pelte) bien que les rivets du revers montrent que l'objet a été utilisé.

163. Bouton émaillé; prosp. et don G. Cabrol; MPM, inv. 993.16.1; diam. 21 mm. Bouton en forme de fleur à 8 pétales, jadis émaillés ainsi que le centre de l'objet. Style du IIIe siècle.

164. Ferret de ceinture, IVe s.; prosp. et don G. Cabrol; MPM, inv. 992.10.7; Feugère 1993a, 253,16; L. act. 37,5 mm.

165. Pendant (harnais?), Ht-Emp.; prosp. et don G. Cabrol; MPM, inv. 992.10.4; L. act. 56 $\mathrm{mm}$. Pendant à crochet, en tôle, mais de forme très inhabituelle: le corps en forme de goutte allongée, orné d'un motif de cercles concentriques, se prolonge par un disque portant le même décor.

- Chemin de Montpeyroux (habitat gallo-romain)

166. Pendant phallique, Ht-Emp.; Schneider, Garcia 1998, fig. 234. Pendant phallique en bronze, associant de manière classique un phallus dressé à droite et une main faisant le geste de la «figue» à gauche, au-dessus de parties génitales masculines.

- ND-de-Figuières (quartier périphérique d'Aulas)

167. Extrémité de pendant; prosp. et don G. Cabrol; MPM, inv. 989.6.1; L. act. 29 mm. Extrémité triangulaire, accostée de festons, d'un objet coulé à bouton terminal, qui semble avoir comporté un élément central en relief; malgré ces caractéristiques originales, qui 


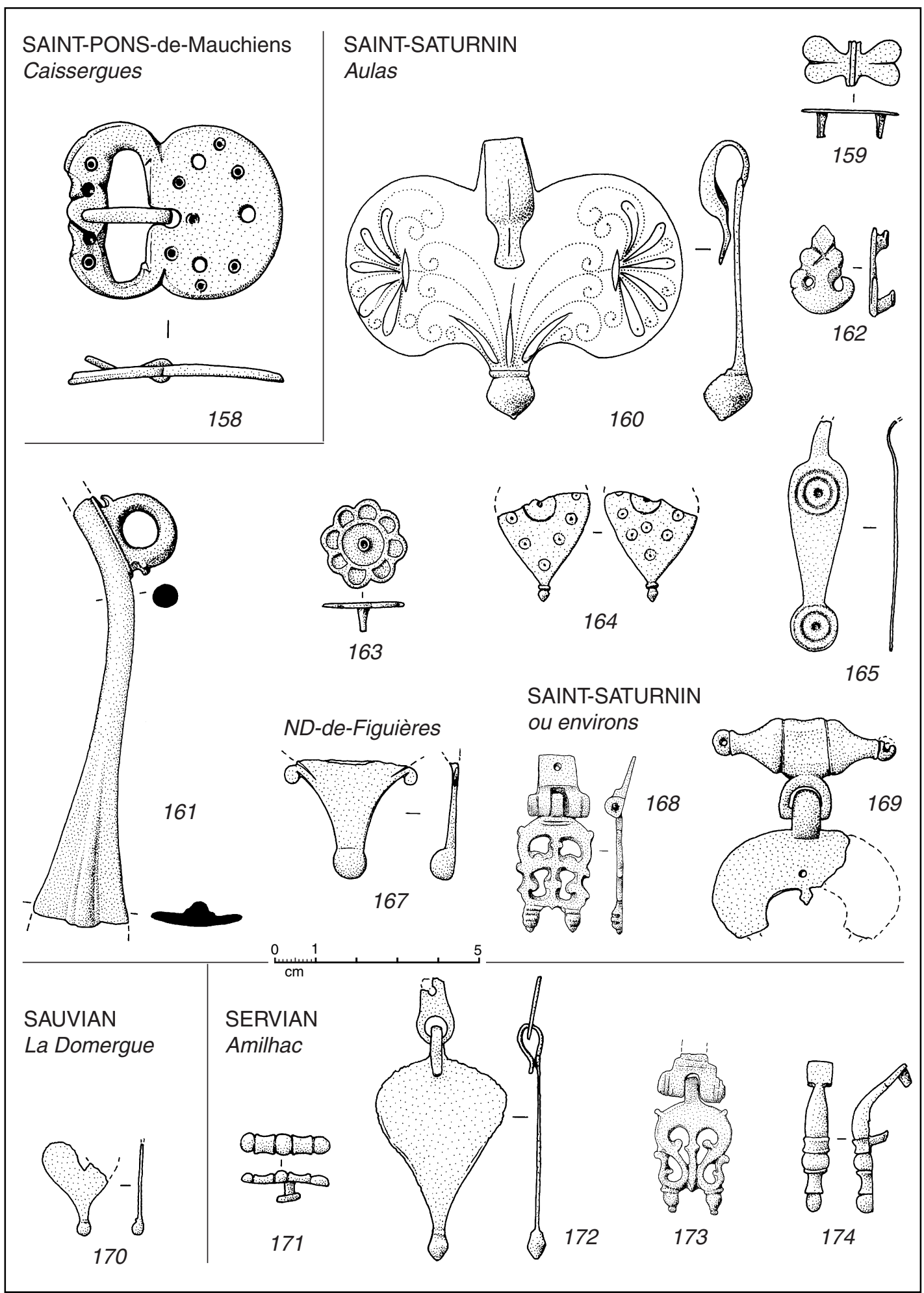

Fig. 18. Militaria du département de l'Hérault (v. le catalogue). Ech. 2/3. 
peuvent faire écarter l'idée d'un pendant, il est difficile de ne pas attribuer cet objet au domaine du harnais.

\section{- St-Saturnin ou environs}

168. Pendant de harnais à charnière; prosp. et don Chr. Berthomieu; MPM, inv. 2000.22.1; L. totale 43,5 mm. Pendant composite, articulé (axe en fer) sur une petite plaque de fixation rectangulaire, percée d'un trou. Le pendant se compose principalement d'une perle ajourée, prolongée vers le bas par deux fleurons et deux lests en forme de glands. Cette forme est attestée localement à Servian.

169. Applique de harnais avec son pendant; prosp. et don Chr. Berthomieu; MPM, inv. 2000.22.2; L. de l'applique $46 \mathrm{~mm}$. Applique de forme allongée aux extrémités percées pour une fixation rivetée sur une lanière horizontale, ayant conservé une partie d'un pendant en forme de lunule, étamé (objet identique à Rißtissen: Ulbert 1970, $\mathrm{n}^{\circ} 347$ ).

Sauvian (Fig. 18)

- La Domergue (grande villa gallo-romaine)

170. Petit pendant; prosp. et don G. Cros; MPM, inv. 995.24.6; L. act. $22 \mathrm{~mm}$. V. ici les découvertes comparables d'Aspiran ( $\left.\mathrm{n}^{\circ} 10\right)$, Lattes $\left(\mathrm{n}^{\circ} 58\right)$ et Mèze $\left(\mathrm{n}^{\circ} 90\right)$.

Servian (Fig. 18 et 19)

- Amilhac (grande villa gallo-romaine)

171. Applique de harnais; prospections et coll. P. Abauzit; L. $22 \mathrm{~mm}$. Type côtelé, à décor symétrique, mais pourvu au revers d'un seul rivet de fixation.

172. Pendant de harnais; prospections et coll. P. Abauzit; L. $57,5 \mathrm{~mm}$. Ce pendant a conservé l'attache caractéristique de ces objets (v. ici à Fontès, $n^{\circ} 48$ ) et Loupian, $n^{\circ} 69$ ); première moitié du Ier s. ap. J.-C.

173. Pendant articulé; prospections et coll. P. Abauzit; L. act. $39 \mathrm{~mm}$. Pendant à charnière ayant conservé une partie de la patte de fixation sur la lanière en cuir. V. ci-dessous le parallèle local de Saint-Saturnin ( $\left.n^{\circ} 168\right)$ et le fragment de Lespignan $\left(n^{\circ} 59\right)$.

174. Pendant de lanière; prosp. et coll. P. Abauzit; L. $37 \mathrm{~mm}$. À comparer avec le pendant de harnais de Fontès, $n^{\circ} 54$.

- St-Adrien (villa gallo-romaine, puis chapelle médiévale)

175. Applique de ceinture, IVe-Ve s.; prosp. et coll. J.-L. Espérou; Depeyrot et al. 1986, fig. 58, b2; L. $26 \mathrm{~mm}$. Applique de ceinture «en forme d'hélice», pourvue de rivets rapportés et non coulés, comme la plaque-boucle de Saint-Pons-de-Mauchiens ( $\left.\mathrm{n}^{\mathrm{o}} 158\right)$.

- sans précision

176. Agrafe de harnais, Ht-Emp.; prosp. et coll. J.-L. Espérou; Depeyrot et al. 1986, fig. 55, b8; L. act. $60 \mathrm{~mm}$. Cette agrafe, d'un type particulier («male strap fastener» de Bishop 1988?) ne semble pas appartenir à un type courant en Gaule du Sud, bien que l'incrustation d'un fil d'argent observé entre deux côtes relève d'une technique bien observée, elle, sur les productions des bronziers rutènes, en particulier les fibules.

177. Pendant de harnais, Ier s.; prosp. et coll. J.-L. Espérou; Depeyrot et al. 1986, fig. 55, b7; L. act. $33 \mathrm{~mm}$. Comme l'exemplaire de Quarante $\left(\mathrm{n}^{\circ} 141\right)$, cet objet appartient à une petite série d'objets probablement fabriqués en Gaule du Sud, influencés par les différents modèles de pendants à charnière (v. à Clermont-l'H., $n^{\circ} 36$ et 37).

Tourbes (Fig. 19)

- Les Demoiselles-Ouest (villa gallo-romaine et atelier de potier) 


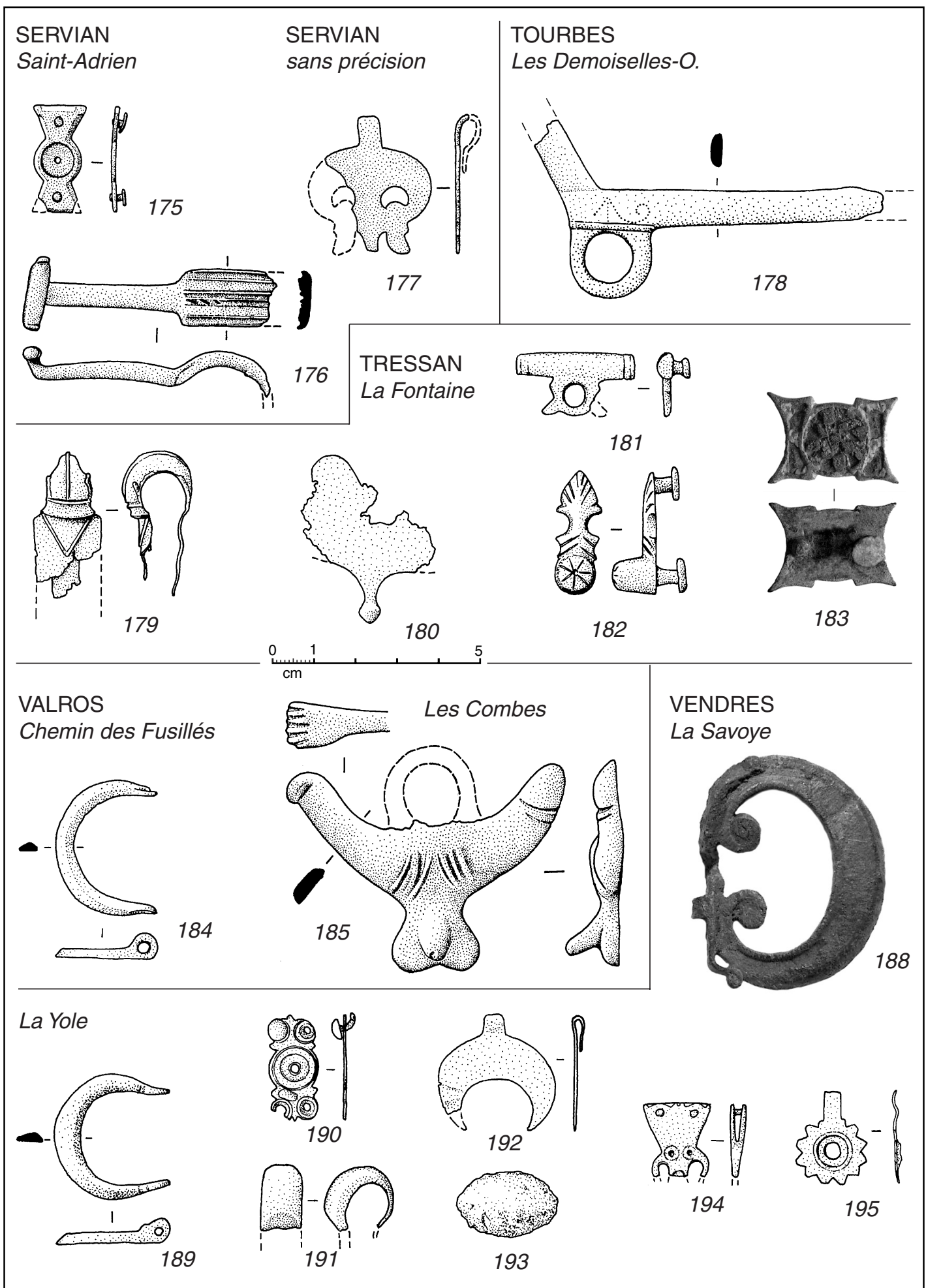

Fig. 19. Militaria du département de l'Hérault (v. le catalogue). Ech. 2/3. 
Tressan (Fig. 19)

- La Fontaine (villa gallo-romaine sur établissement indigène)

179. Agrafe de harnais, Ht-Emp.; prosp. et don H. Leyris; MPM, inv. 990.28.8; L. act. 35,5 $\mathrm{mm}$. Le profil du dos évoque celui de l'arc de fibules augustéennes (type F.16).

178. Fragment de caveçon en bronze, Ht-Emp.; prosp. et don G. Cabrol, 1995; MPM, inv. 995.49.1. Objet de facture délicate, avec les restes d'un décor de volutes en fines ponctuations. Cet objet est probablement plus précoce que l'autre fragment de caveçon antique du département, celui de Saint-Saturnin (ci-dessous, $\mathrm{n}^{\circ} 161$ ).

180. Pendant de harnais; prosp. et don H. Leyris; MPM, inv. 990.28.25. L. act. 41,5 mm. Petit pendant de forme usuelle, dont le crochet manque; première moitié du Ier $\mathrm{s}$.

181. Applique de harnais; prosp. et don H. Leyris; MPM, inv. 991.21.3; L. 29 mm. Barrette d'applique, à deux rivets, se distinguant de la forme courante par la présence d'un anneau latéral, accosté de deux ergots. Ces petits décors montrent occasionnellement des appendices de formes diverses (par ex. Böhme 1978, B162 et B163), mais cet exemplaire constitue en fait l'attache d'un pendant coulé, comme le montre un exemplaire complet de Bordeaux (fig. 6, 2).

182. Applique de harnais, Ht-Emp.; prosp. et don H. Leyris; MPM, inv. 995.75.2; L. 29,5 $\mathrm{mm}$. Applique à deux forts rivets, en forme de feuille échancrée, prolongée par un bouton massif, lui aussi marqué d'incisions.

183. Applique émaillée; prosp. et don H. Leyris; MPM, inv. 995.75.3; L. 31,5 mm. Les trois parties de l'objet étaient ornées d'émaux composites (millefiori), partiellement conservés: dans chacun des deux motifs à bords concaves des extrémités, semis de fleurs bleues à cœur rouge, sur fond blanc; au centre, damier de pavés quadrangulaires, alternés et séparés par des traits rouge: blanc -bleu; et noir- blanc dans un cadre bleu. La complexité de ces motifs, tout comme la forme de l'objet, trouve des parallèles au IIIe s. de n. ère: harnais de Wange (Lodewijckx 1993, fig. 5, 4.4); pour le Midi, où ce type est rare, on citera une découverte de Cabrières d'Aygues (Vaucluse), acquise en 1867 par le Musée Calvet d'Avignon (inv. J.443p).

Valros (Fig. 19)

- Ch. Des Fusillés (habitat gallo-romain)

184. Boucle de harnais, Ier-IIe s.; prosp. et don G. Cros; MPM, inv. 2001.24.1; L. 32 mm.

- Les Combes (habitat gallo-romain)

185. Pendant phallique, Ht-Emp.; Depeyrot et al. 1986, fig. 61,1

Vendres (Fig. 19)

- Montée de Jaussan (habitat gallo-romain)

186. Agrafe de harnais, Ht-Emp.; prospections et coll. P. Abauzit.

- La Savoye (villa gallo-romaine)

188. Boucle de cingulum, Ier s.; Musée du Biterrois, inv. 12.10.88; L. 56 mm. Boucle dont les volutes rentrantes devaient épauler un ardillon à ergots; l'axe de la charnière était également prolongé par de petites volutes externes (cf. Rißtissen: Ulbert 1970, $\mathrm{n}^{\circ}$ 506).

- La Yole (villa gallo-romaine)

189. Boucle de harnais, Ier s.; prospections et coll. P. Abauzit; $30 \times 28 \mathrm{~mm}$. Pas trace de l'axe, qui pouvait donc être en bronze.

190. Applique de harnais, Ier s.; prospections et coll. P. Abauzit, 2002; L. 25 mm. Petite applique qui devait comporter un bouton central riveté; les fortes moulures autour des 


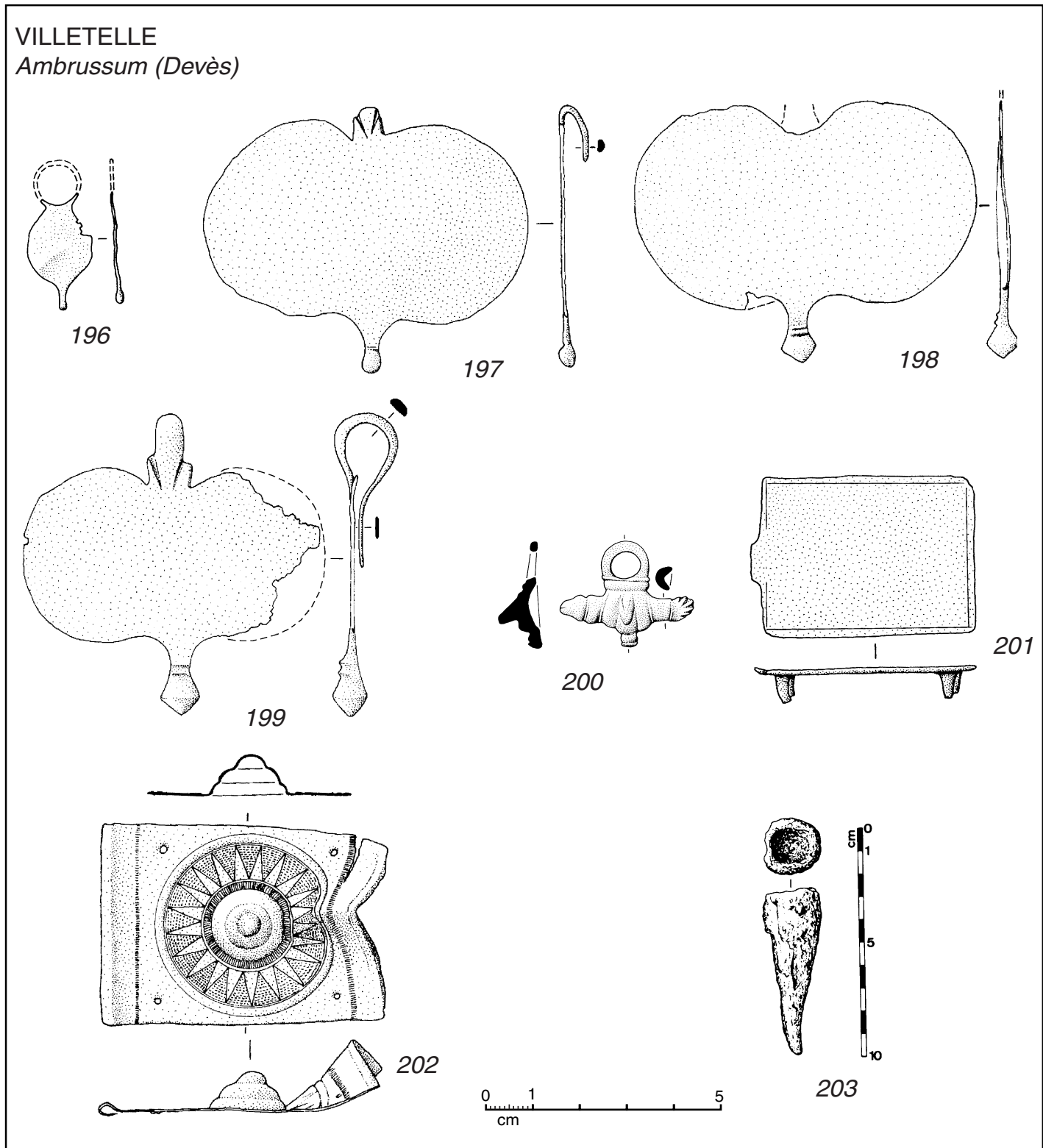

Fig. 20. Militaria du département de l’Hérault (v. le catalogue). Ech. 2/3.

4 rivets de fixation (l'un d'eux est conservé) sont caractéristique d'un style de décors de harnais du Ier $\mathrm{s}$. attesté localement à Neffiès $\left(\mathrm{n}^{\circ} 111\right)$ et Pomerols $\left(\mathrm{n}^{\circ} 126\right)$.

191. Fragment d'agrafe de harnais; prospections et coll. P. Abauzit; L. act. 18 mm.

192. Lunule en bronze; prospections et coll. P. Abauzit; L. 27,5 mm. Des pendants en forme de lunule apparaissent sur certaines figurations de harnais militaire (stèle de T. Flavius Bassus: Feugère 1993a, 176), mais compte tenu de l'aspect apotropaïque de ce motif, on ne peut exclure d'autres usages civils.

193. Balle de fronde (?) en plomb; prospections et coll. P. Abauzit; L. $24 \mathrm{~mm}$, poids 30,90 g. La forme, ramassée, est inhabituelle pour ce type de projectile. 
194. Ferret de ceinture, IVe-Ve s.; prospections et coll. P. Abauzit; L. act. $18 \mathrm{~mm}$. Sommet d'un ferret coulé en forme d'amphore, présentant le décor d'ocelles particulièrement prisé à l'époque, notamment dans la région.

195. Applique de ceinture, IVe-Ve s.; prospections et coll. P. Abauzit; L. act. 21,5 mm. Applique en tôle, à languette; pourtour dentelé. Il semble s'agir d'une variante locale des appliques fréquemment attestées sur les ceintures des tombes à mobilier «germanique» de Gaule septentrionale, où le pourtour est en général discrètement guilloché; mais on rencontre également des contours découpés en dents de scie, dont peut découler ce type d'applique (Böhme 1974, pl. 46, 20)

Villetelle (Fig. 20-22)

- Ambrussum, Le Devès (important habitat protohistorique occupé jusqu'au Ier s. de n. ère)

196. Pendant foliacé, contexte Ier s. ap. J.-C.; Feugère 1986, fig. 88, 77; L. act. 26 mm. Petit pendant de harnais foliacé, dont la suspension est dégagée dans la masse: une particularité déjà rencontrée ici à Nissan $\left(\mathrm{n}^{\circ} 117\right)$ et au Pouget $\left(\mathrm{n}^{\circ} 130\right)$.

197. Pendant de harnais, contexte Ier s. ap. J.-C.; Feugère 1986, fig. 89b; L. 58, larg. 69 $\mathrm{mm}$.

198. Pendant de harnais, contexte Ier s. ap. J.-C.; Feugère 1986, fig. 89c; L. act. 57, larg. $72,5 \mathrm{~mm}$.

199. Pendant de harnais, contexte Ier s. ap. J.-C.; Feugère 1986, fig. 89d; L. 66, larg. 63 $\mathrm{mm}$.

200. Pendant phallique, c. 2b, contexte Ier s. ap. J.-C.; Feugère 1986, fig. 78, 13; L. 45 mm. Pendant plus massif et trapu que la plupart des découvertes de ce type.

201. Plaque de cingulum, P4, c.3, contexte Ier s. ap. J.-C.; Feugère 1986, fig. 88, 76; 48,5 x $34 \mathrm{~mm}$. Bien que dépourvu de tout décor à l'exception d'un filet de bordure, ce type de plaque est généralement rattaché au harnachement (Rißtissen: Ulbert 1970, pl. 24, 360362) ou cingulum militaire (Augst: Fünfschilling 1993, $\mathrm{n}^{\mathrm{o}} 1108$ ).

202. Plaque de cingulum, contexte Ier s. ap. J.-C.; Feugère 1986, fig. 89a; 62 x 44 mm. Applique de cingulum d'infanterie, de forme classique, illustrée tant par certains reliefs funéraires que par d'innombrables découvertes sur les sites militaires du limes.

203. Talon de lance, contexte Ier s. ap. J.-C.; Feugère 1986, fig. 88, 74; L. 74 mm. V. les observations faites à propos du talon de Montagnac, $\mathrm{n}^{\circ}$ 99).

- Ambrussum, Le Sablas (quartier bas de l'oppidum)

204. Deux pendants à lunule, contexte 10 av. / 10 ap. J.-C.; Feugère, Tendille 1989, no 176177; L. 165 et $192 \mathrm{~mm}$. Ces deux objets complexes, formant une paire, ont été publiés en 1989 sans parallèles; nous n'en connaissons pas plus aujourd'hui, si ce n'est pour les seuls pendants, eux-mêmes pourvus d'un pendentif foliacé articulé entre les branches des lunules (Bishop 1988: type 9h).

205. Agrafe de harnais, contexte 10 av. / 10 ap. J.-C.; Feugère, Tendille 1989, nº 175; L. 25 $\mathrm{mm}$.

206. Agrafe de harnais, contexte 50/75 ap. J.-C.; Feugère, Tendille 1989, nº 252; L. 55 mm.

207. Bouton de harnais, contexte 50/75 ap. J.-C.; Feugère, Tendille 1989, nº 253; diam. 35 $\mathrm{mm}$.

208. Applique de harnais, contexte 75/100 ap. J.-C.; Feugère, Tendille 1989, nº 284; L. act. $16 \mathrm{~mm}$.

209. Bouton à anneau, contexte 10 av. / 10 ap. J.-C.; Feugère, Tendille 1989, nº 174; L. 21 $\mathrm{mm}$. La barre transversale ainsi que la boucle triangulaire correspondent à l'une des formes les plus précoces de ces objets, ce que confirme le contexte. Une variante à bouton rond a été recueillie à Ensérune (ci-dessus $n^{\circ} 116$ ). 


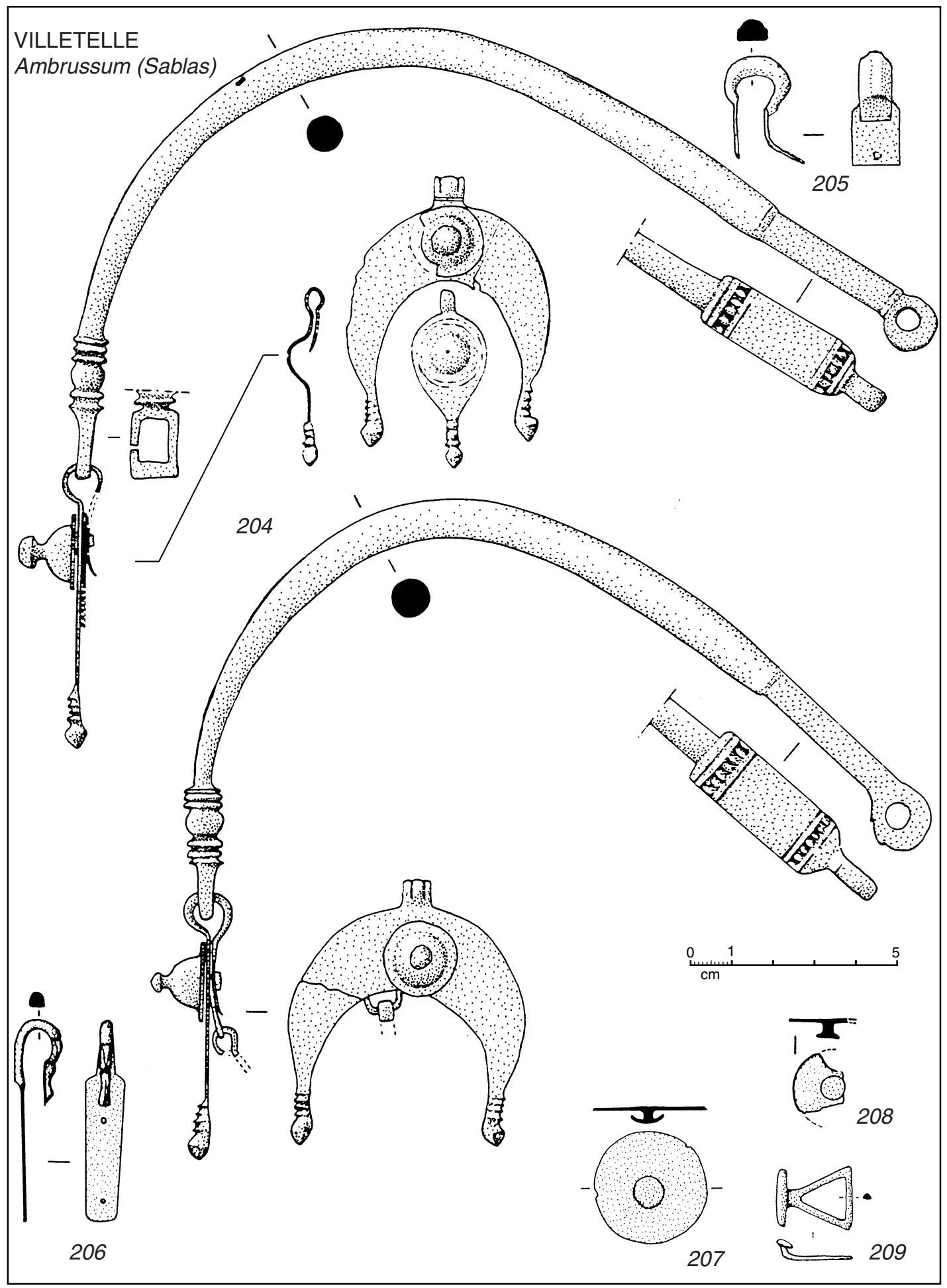

Fig. 21. Militaria du département de l'Hérault (v. le catalogue). Ech. 2/3. 
210. Mors constitué d'une partie du mors brisé en fer et d'une applique latérale en bronze, à attache rectangulaire largement ouverte; fouilles J.-L. Fiches, période VII, 180-230 ap. J.-C. (Feugère, Tendille 1989, 160, fig. 115, nº 337).

211. Applique latérale d'un mors, en bronze, attache rectangulaire à étroite ouverture carrée; fouilles J.-L. Fiches, période VII, 180-230 ap. J.-C.; Feugère, Tendille 1989, 160, fig. $115, \mathrm{n}^{\circ} 338$.

212. Applique latérale d'un mors, en bronze, avec départ de l'attache rectangulaire; fouilles J.-L. Fiches, période VII, 180-230 ap. J.-C.; Feugère, Tendille 1989, 160, fig. 115, n 339.

213. Bouton de harnais, contexte 120/180 ap. J.-C.; Feugère, Tendille 1989, ñ 319; diam. 12 $\mathrm{mm}$. Tête creuse, à rapprocher de l'extrémité de lanière de Loupian, $\mathrm{n}^{\mathrm{0}} 62$.

214. Pendant foliacé, contexte 70/100 ap. J.-C.; Feugère, Tendille 1989, nº 282; L. act. 39 $\mathrm{mm}$.

215. Pendant de harnais (crochet), contexte 75/100 ap. J.-C.; Feugère, Tendille 1989, $\mathrm{n}^{\mathrm{o}}$ 281 ; L. act. $58 \mathrm{~mm}$.

216. Pendant de harnais (crochet), contexte 75/100 ap. J.-C.; Feugère, Tendille 1989, ${ }^{\circ}$ 283; L. act. $82 \mathrm{~mm}$ (Bishop 1988: type 3a).

217. Plaque de cingulum? contexte 75/100 ap. J.-C.; Feugère, Tendille 1989, nº 274; L. 44mm. Plaque avec décor poinçonné et gravé dans les angles, 4 trous de fixation.

Vallée de l'Hérault, sans précision (Fig. 22)

218. Applique de harnais; prosp. et don Ch. Berthomieu; MPM, inv. 2000.81.1; L. $20 \mathrm{~mm}$. Applique en forme de coquille.

\section{Département de l'Hérault, provenance inconnue (Fig. 22)}

219. Pendant de harnais, Ht-Emp.; Musée de Montpellier; L. 64,5mm. Sur ce pendant martelé, à umbo central, le motif phallique placé à la base est extrêmement stylisé, et dépourvu de pénis central. Le meilleur parallèle héraultais est fourni par la découverte de Nissan, $\mathrm{n}^{\mathrm{o}} 118$, d'une figuration un peu plus complète, mais tout aussi schématique.

220. Boucle de type Teba, IVe s.; Feugère 1993a, 253, 12; 1996b, 277, nº 12; Musée de Montpellier; L. 34 mm. V. ci-dessus à propos de l'exemplaire de Frontignan, $n^{0} 55$.

221. Plaque-boucle de type Tirig, IVe s.; Feugère 1993a, 253, 3; 1996b, 277, $\mathrm{n}^{\circ} 3$; Musée de Montpellier; L. $48 \mathrm{~mm}$. Un excellent parallèle régional est fourni par une plaqueboucle d'une inhumation de Saint-Clément (Gard), ayant conservé sa boucle à dauphins affrontés (Feugère 1996b, 277, $\mathrm{n}^{\circ}$ 2). 


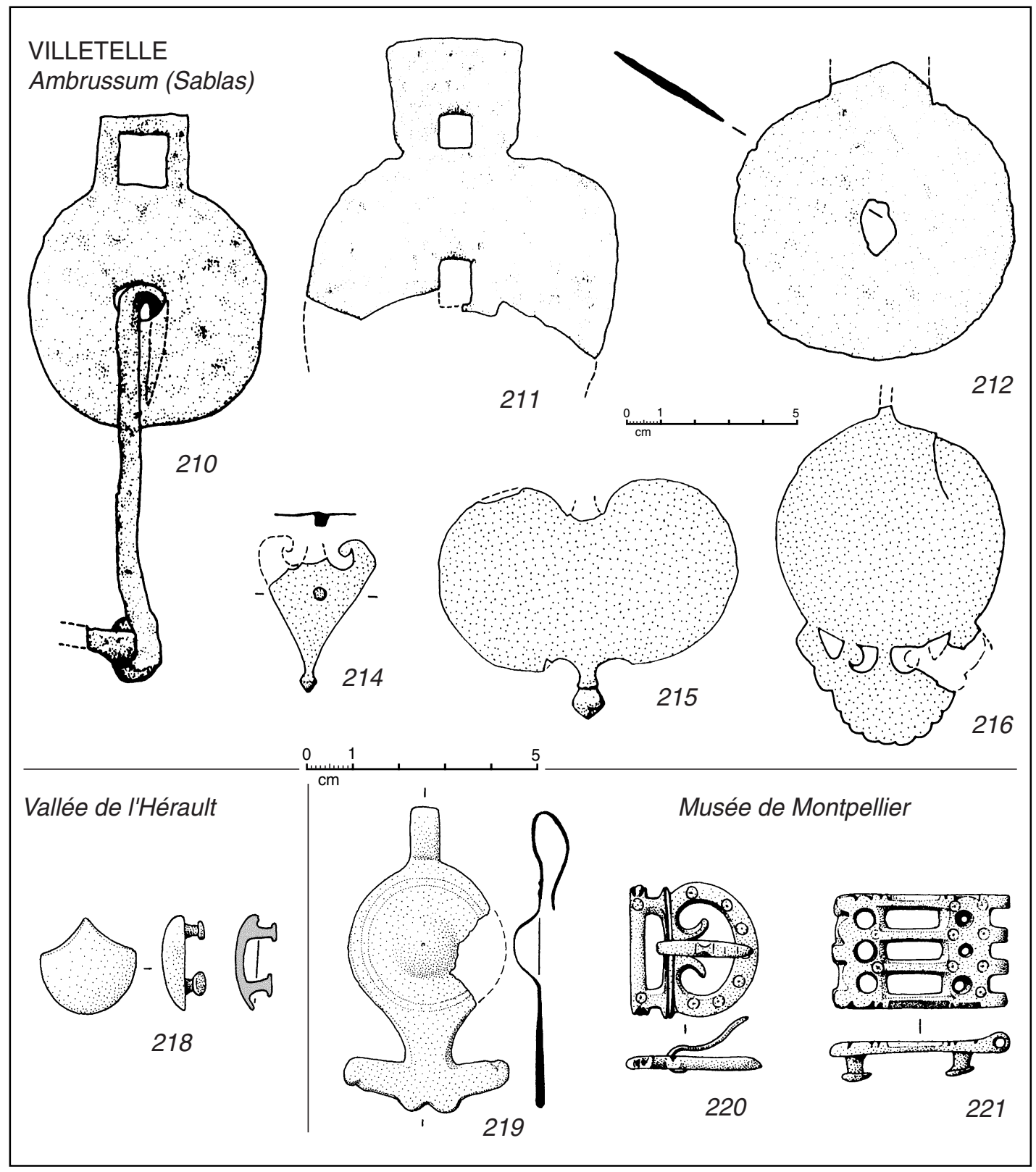

Fig. 22. Militaria du département de l'Hérault (v. le catalogue). Ech. 2/3. 


\section{BIBLIOGRAPHIE}

Abréviations:

BAR: British Archaeological Reports

BSESS: Bulletin de la Société d'Etudes de Sète et sa région

JRMES: Journal of Roman Military Equipment Studies

RAN: Revue Archéologique de Narbonnaise

RSL: Rivista di Studi Liguri

Abascal, Sanz (1993): J.M. Abascal Palazón, R. Sanz Gamo, Bronces antiguos del Museo de Albacete, Albacete 1993.

ABAuzit (2000): P. Abauzit, Décors d'applique à bordure ajourée de Gaule méridionale. Instrumentum 11, juin 2000, 16-17.

Allason-Jones (1988): L. Allason-Jones, 'Small finds' from turrets on Hadrian's Wall. In: J.C. Coulston (ed.), Military equipment and the Identity of Roman Soldiers. Proceedings of the Fourth Military Equipment Conference (BAR S-394), Oxford 1988, 197-233.

AMBARD et al. (1972): R. Ambard, G. Bertucchi, J.-M. Gassend, Fouilles d'urgence et découverte du decumanus à Aix-en-Provence. RAN V, 1972, 31-47.

Arnaud (1999): P. Arnaud, D. Boisse, J. Gautier, Militaria de Gaule méridionale, 12. Balles de fronde antiques en plomb du pays Salluvien, Cavare et Voconce (Bouches-du-Rhône). Instrumentum $\mathrm{n}^{\mathrm{o}}$ 9, juin 1999, 26-28.

ARrAmond et al. (1986): J.-C. Arramond et al., Eauze. Témoins archéologiques de l'antique cité des Elusates, Sorèze 1986.

AurRecoecheA (2001): J. Aurrecoechea Fernández, Los cinturones romanos en la Hispania del Bajo Imperio (Monogr. Instrum. 19), Montagnac 2001.

BAATZ (1991): D. Baatz, Die römische Jagdarmbrust. Arch. Korrespondenzblatt 21 (2), 1991, 283-290.

BARCOCZI (1946/48): L. Barcoczi, Arch. Ertesitö série III, vol. VIII-IX, 1946/48, 169-182.

BARRUOL, SAUZADE (1969): G. Barruol, G. Sauzade, Une tombe de guerrier à Saint-Laurent-desArbres, Gard, contribution à l'étude des sépultures du Ier s. av. J.-C. dans la basse vallée du Rhône, $R S L, 35,1969,15-89$.

BATs (1990): M. Bats, Tombes et nécropoles de Narbonnaise aux IIe-Ier s. av. J.-C.: problèmes de datation et de chronologie. In: A. Duval, J.-P. Morel et Y. Roman (dir.), Gaule interne et Gaule méditerranéenne aux IIe et Ier siècles avant J.-C.: confrontations chronologiques. Actes de la tableronde de Valbonne, 1986 (Suppl. 21 à la Rev. Arch. Narb.), Paris 1990, 269-289.

Bishop (1988): M.C. Bishop, Cavalry equipment of the Roman army in the first century AD. In: J.C. Coulston (ed.), Military equipment and the Identity of Roman Soldiers. Proceedings of the Fourth Military Equipment Conference (BAR S-394), Oxford 1988, 67-196.

BISHOP (1992): M.C. Bishop, The early imperial 'apron'. JRMES 3, 1992, 81-104.

BisHop (1996): M.C. Bishop, Finds from Roman Aldborough. A Catalogue of Small Finds from the Romano-British Town of Isurium Brigantum (Oxbow Monographs 65), Oxford 1996.

BISHOP (1999): M. Bishop, The Newstead «lorica segmentata». JRMES 10, 1999, 27-43.

BöHME (1978): A. Böhme, Metallfunde. In: H. Schönberger et al., Kastell Oberstimm. Die Grabungen 1968-1971 (Limesforschungen, 18), Berlin 1978, 164-227.

Bonnin (1860): Th. Bonnin, Antiquités gallo-romaines des Eburoviques, publiées d'après les recherches et les fouilles dirigées par M. Th. Bonnin, Paris 1860.

Boschung (2000): D. Boschung, Figürliche Kleinbronzen aus Xanten. Ein konventionelle Bilderwelt und ihre Quellen. Kölner Jahrb. 33, 2000, 121-130.

Boube (1980): Chr. Boube-Piccot, Les bronzes antiques du Maroc, 3. Les chars et l'attelage, Rabat 1980 . 
Boube (1994): Chr. Boube-Piccot, Les bronzes antiques du Maroc, 4. L'équipement militaire et l'armement, Paris 1994.

Bouchette et al. (1998): A. Bouchette, B. Boulestin, J.-R. Bourhis, J.-F. Buisson, C. Duffault, J. Gomez de Soto, S. Lemoine, J.-F. Tournepiche, C. Vernou, M. Vieau, M. Coutureau, Le char romain du Musée Archéologique de Saintes, Saintes 1998.

BOUdET (1996): R. Boudet, Rituels Celtes d'Aquitaine (préf. de J. Guilaine), Paris 1996.

BO•I• (2001): D. Bo•••, Décors de harnais romain à bordure ajourée. Instrumentum 13, 2001, 25-26.

CATHALA (1903): M. Cathala, Note sur un cimetière gallo-romain et sur les trouvailles faites dans la commune d'Argeliers au cours de travaux d'adduction d'eau, en 1902. Bull. Soc. Et. Scient. Aude XIV, 1903, 189 sqq.

Снавот, Feugère (1993): L. Chabot, M. Feugère, Les armes de l'oppidum de la Cloche (Les Pennes-Mirabeau, B.-du-Rh.) et la destruction du site au Ier siècle avant notre ère. Doc. Arch. Mérid. 16, 1993, 337-351.

DAwson (1990): M. Dawson, Roman military equipment on civil sites in Roman Dacia. JRMES 1, 1990, 7-15.

DESCHLER-ERB, Schwarz (1993): E. Deschler-Erb, P.-A. Schwarz, A bronze spearhead from Insula 22, and its significance for the urban history of Augusta Rauricorum (Augst BL, Switzerland). JRMES 4, 1993, 9-22.

DESCHLER-ERB (1998a): E. Deschler-Erb, «Geflügelte» Pferdegeschirranhänger. In: R. Ebersbach u. A.R. Furger (Hrsg.), Mille Fiori. Festschr. L. Berger (Forsch. Augst 25), Augst 1998, 115-122.

DESCHLER-ERB (1998b): E. Deschler-Erb, Geflügelte Pferdegeschirranhänger (Bishop Typ 7): eine Bibliographie. Instrumentum 7, juin 1998, 14-15.

DesChleR-ERB (1999a): E. Deschler-Erb, Ad arma! Römisches Militär des 1. Jahrhunderts $n$. Chr. in Augusta Raurica (Forsch. in Augst 28), Augst 1999.

Deschler-ERB (2000): E. Deschler-Erb, Niellierung auf Buntmetall: ein Phänomen der frühen Kaiserzeit. Kölner Jahrb. 33, 2000, 383-396.

EGG, Pare (1995): M. Egg, Ch. Pare, Die Metallzeiten in Europa und im vorderen Orient. Die Abteilung Vorgeschichte im römisch-Germanischen Zentralmuseum (Kat. vor- unf frühgeschichtl. Altertümer, 26), Mainz 1995.

FAUduet (1992a): I. Fauduet, Musée d'Evreux. Collections archéologiques. Bronzes galloromains. Instrumentum, Evreux, 1992.

FeugÈRe et al. (1987): M. Feugère, J.-P. Joly, C. Pellecuer et A. Peyre. La nécropole galloromaine tardive de La Brèche (Laudun, Gard), Premières données. Arch. en Lang. 1987 (4), 81-89.

FeUGĖRE (1981): M. Feugère, Découvertes au quartier de Villeneuve, Fréjus, Var: le mobilier métallique et la parure. Doc. Arch. Mérid. 4, 1981, 137-168.

FeUGÈre (1983): M. Feugère, L'équipement militaire romain dans le département de la Loire. Contribution à l'étude de la romanisation en pays ségusiave. Cah. Arch. Loire 3, 1983, 45-66.

FeUGĖRE (1992): M. Feugère, Les instruments de chasse, de pêche et d'agriculture. In: M. Py (dir.), Recherches sur l'économie vivrière des Lattarenses (Lattara 5), Lattes 1992, 139-162.

FeUGÈRE (1993a): M. Feugère, Les armes des Romains, de la République à l'Antiquité tardive, Paris 1993.

FEUGÈRE (1993b): M. Feugère, L'évolution du mobilier non céramique dans les sépultures antiques de Gaule méridionale (IIe siècle av. J.-C. - début du Ve siècle ap. J.-C.). In: M. Struck (Hrsg.), Römerzeitliche Gräber als Quellen zu Religion, Bevölkerungsstruktur und Sozialgeschichte (Arch. Schr. des Inst. für Vor- und Frühgeschichte der J.-G. Universität Mainz, 3), Mainz 1993, 119-165.

FeugÈRe (1994a): M. Feugère, Montagnac. Activités et acquisitions de la Maison du Patrimoine en 1993 (Cah. du Patrimoine, 1), Montagnac 1994.

FeugÈRE (1994b): M. Feugère, Casques antiques. Les visages de la guerre, de Mycènes à la fin de l'Empire romain, Paris 1994. 
FEUGÈRE (1996a): M. Feugère, Militaria de Gaule méridionale, 2. Applique de mors d'Espondeilhan (Hérault). Arma 8 (1-2), 1996, 5-6.

FEUGÈRE (1996b): M. Feugère, L'armement du Bas-Empire. In: M. Reddé (dir.), L'armée romaine en Gaule, Paris 1996, 267-278.

FEugÈRE (1997): M. Feugère, Militaria de Gaule méridionale, 4. Vaison-la-Romaine (Vaucluse). Arma 9, 1997, 3-6.

Feugère, Pellecuer (1998): M. Feugère, Chr. Pellecuer, Militaria de Gaule méridionale, 7: Mèze (Hérault) et environs. Arma 10, 1-2, 1998, 5-9.

Feugère, Pillard (1999): M. Feugère, J.-P. Pillard, Militaria de Gaule méridionale, 11. Alleins, «La Tamberlette» (Bouches-du-Rhône). Instrumentum 9, juin 1999, 25-26.

FeugÈRe, Poux (2011): M. Feugère, M. Poux, Gaule pacifiée, Gaule libéreé? Enquête sur les militaria en Gaule civile. Ges. Pro Vindonissa Jahresber. 2001, (Romec XIII, 2001), 79-95.

FINGERLIN (1986): G. Fingerlin, Dangstetten I, Katalog der Funde (Fundstellen 1 bis 603), Stuttgart, 1986.

FrERE (1984): S. Frere, Verulamium Excavations, vol. III, Oxford 1984.

FünfSCHILling (1993): S. Fünfschilling, Römische Altfunde von Augst-Kastelen. Eine Privatsammlung mit Altfunden aus Augusta Raurica [...] (Interne Augster Arbeitspapiere, 2), Augst 1993.

HÉRON DE VILlefosse (1903): A. Héron de Villefosse, Ceinturon romain découvert à Argeliers (Aude). Bull. Archéol. 1903, 64-67.

Hosdez, Jacques (1989): Ch. Hosdez, A. Jacques, La nécropole à incinérations de Baralle (Pasde-Calais) (Nord-Ouest Archéol., 2), s.1., 1989.

JACOBI (1974): G. Jacobi, Werkzeug und Gerät aus dem oppidum von Manching (Die Ausgr. in Manching, 5), Wiesbaden, 1974.

JAHN (1860): O. Jahn, Die Lauersforter Phalerae, Bonn 1860.

JAMES (1977): E. James, The Merowingian Archaeology of South West Gaul (BAR), Oxford 1977.

JÜTTING (1995): I. Jütting, Die Kleinfunde aus dem römischen Lager Eining-Unterfeld. Bayer. Vorgeschichtsbl. 60, 1995, 143-230.

LAPEyre (1987-88): Cl. Lapeyre, Béziers, fouilles anciennes. Bull. Soc. Arch. Scient. Litt. Béziers $6^{\mathrm{e}}$ sér., 3, 1987-1988, 9-29.

LAURIOL (1964-65): J. Lauriol, Premières notes sur la nécropole du «Pech» (Minerve, hérault). Bull. Soc. Et. Scient. Aude LXV, 1964-65, 187.

LE BoHeC (1989): Y. Le Bohec, L'armée romaine, Paris 1989.

LODEWIJCKX (1993): M. Lodewijckx et al., A third century collection of decorative objects from a Roman villa at Grange (Central Belgium): first interdisciplinary report. JRMES 4, 1993, 67-99.

LouIs (1953): M. Louis, Un casque du IIe Age du Fer à Montpellier. Gallia XI, 1953, 306-307.

LugAnd, Bermond (dir.) (2001): M. Lugand, I. Bermond (dir.), Agde et le Bassin de Thau (Carte Archéologique de la Gaule, fasc. 34/2), Paris 2001.

LuIK (1996): M. Luik, Köngen-Grinario I. Topographie, Fundstellenverzeichnis, ausgewählte Fundgruppen (Forsch. und Ber. z. Vor- und Frühgeschichte in B.-W. 62), Stuttgart 1996.

MACKENSEN (2001): M. Mackensen, Militärische oder zivile Verwendung frühkaiserzeitlicher Pferdegeschirranhänger aus der Provinz Africa Proconsularis und den Nordwestprovinzen. Germania 79, 2001, 325-346.

Majurel et al. (1973): R. Majurel, J. Ménager, H. Prades, L'habitat et la nécropole de SaintMichel (commune de Montpellier. Ogam, 22-25, 1970-1973, 49-124.

MAQUART (1936): M. Maquart, Note sur les moules à fibules découverts à Nandin (ChâteauPorcien, Ardennes). Bull. Soc. Arch. Champenoise 1-4, 1936, 18-25.

MARIËN (1994): M.-E. Mariën, Quatre tombes romaines du IIIe siècle. Thorembais, Saint-Trond et Overhespen (Monogr. d'archéol. rég., 8), Bruxelles 1994. 
MASSART (2000): Cl. Massart, Eléments de char et de harnachement dans les tumulus tongres du IIIe siècle. Les deux harnachements du tumulus de Celles (Waremme), Belgique. Kölner Jahrb. 33, 2000, 509-522.

MAXFIELD (1981): V. Maxfield, The military decorations of the Roman army, London 1981.

OBMAnN (1999): J. Obmann, Waffen - Statuszeichen oder alltäglicher Gebrauchsgegenstand? In: H. von Hesberg (Hrsg.), Das Militär als Kulturträger in römischer Zeit, Köln 1999, 189-200.

OliviER, Rogers (1978): A. Olivier, G. Rogers, Le monument de Vaugrenier (Alpes-Maritimes). RAN XI, 1978, 143-193

Palol (1955-56): P. de Palol, Pasadores en T iberoromanos en la Péninsula ibérica. Ampurias XVII-XVIII, 1955-56, 97-106.

PASSELAC (1968): M. Passelac, Le mobilier gallo-romain de la grotte du Cimetière à SallèlesCabardès, Aude (Atacina 2), Carcassonne 1968.

Pellecuer (1986): C. Pellecuer, Eléments sur l'Antiquité tardive autour du bassin de Thau, La nécropole du Chemin des Romains à Frontignan (Hérault). BSESS, XIV-XV, 1986, 7-14.

PetCulescu (1995): L. Petculescu, Military equipment graves in Roman Dacia. In: C. van DrielMurray (ed.), Roman Military Equipment: experiment and reality (JRMES 6), 1995, 105-145.

Poux (1999): M. Poux, Puits funéraire d'époque gauloise à Paris (Sénat). Une tombe d'auxiliaire républicain dans le sous-sol de Lutèce (Protohist. Europ. 4), Montagnac 1999.

QuesadA (1997): F. Quesada Sanz, Montefortino-type and related helmets in the Iberian Peninsula: a study in archaeological context. In: M. Feugère (dir.), L'équipement militaire et l'armement de la République (IVe-Ier s. avant J.-C.). JRMES 8, 1997 [1999], 151-166.

RABEISEN (1990): E. Rabeisen, La production d'équipement de cavalerie au 1er s. après J.-C. à Alésia (Alise-Ste-Reine, Côte-d'Or, France). JRMES 1, 1990, 73-98.

RAJTÀR (1994): J. Rajtàr, Waffen und Ausrüstungsteile aus dem Holz-Erde-Lager von Iža. JRMES 5, 1994, 83-95.

SCHAAFF (1988): U. Schaaff, Etruskisch-römische Helme. In: Antike Helme. Handbuch mit Katalog, Mainz 1988, 318-326.

SCHUlten (1927): A. Schulten, Numantia III, München 1927.

SOMMER (1984): M. Sommer, Die Gürtel und Gürtelbeschläge des 4. und 5. Jahrhunderts im römischen Reich (Bonner Hefte zur Vorgeschichte 22), 1984.

STARY (1994): P.F. Stary, Zur eisenzeitlichen Bewaffnung und Kampfesweise auf der Iberischen Halbinsel (Madrider Forsch., 18), Mainz 1994, 2 vol.

Ulbert (1970): G. Ulbert, Das römische Donau-Kastell Rißtissen, Teil 1. Die Funde aus Metall, Horn und Knochen, Stuttgart 1970.

Ulbert (1984): G. Ulbert, coll. H. J. Hildebrands, M. Blech, Cáceres el Viejo. Ein spätrepublikanisches Legionslager in Spanisch-Extremadura (Madrider Beitr. 11), Mainz 1984.

UnZ, Deschler-Erb (1997): Ch. Unz, E. Deschler-Erb, Katalog der Militaria aus Vindonissa (Veröff. Ges. Pro Vindonissa 14), Brugg 1997.

VAn Endert (1991): D. Van Endert, Die Bronzefunde aus dem Oppidum von Manching. Kommentierter Katalog (Die Ausgr. in Manchging, 13), Stuttgart 1991.

Villaverde (1993): N. Villaverde Vega, A prpósito de unos pasadores en forma de «T»: iberroromanos localizados en Carteia (San Roque, Cádiz) y en Septem Fratres (Ceuta). Espacio, Tiempo y Forma (Madrid) ser. II, 6, 1993, 399-418.

WAURICK (1990): G. Waurick, Helme in Caesars Heer, Mainz 1990.

WeBster (1982): G. Webster, Gazetteer of military objects from Cirencester. In: J. Wacher, A. Mc Guirr (eds.), Early Roman occupation at Cirencester (Cirencester Exc., I), Cirencester 1982, 108-117.

Willaume (1987): M. Willaume, Les objets de la vie quotidienne. In: Archéologie d'Entremont au Musée Granet, Aix-en-Pce 1987, 107-142.

ZANIER (1988): W. Zanier, Römische Dreiflügelige Pfeilspitzen. Saalburg Jahrb. 44, 1988, 5-27. 